Florida International University FIU Digital Commons

$10-15-2003$

\title{
Modified diamond dipole antenna for ultra wideband communications using finite difference time domain method
}

Srinivas Desamsetty

Florida International University

DOI: $10.25148 /$ etd.FI14062248

Follow this and additional works at: https://digitalcommons.fiu.edu/etd

Part of the Electrical and Electronics Commons

\section{Recommended Citation}

Desamsetty, Srinivas, "Modified diamond dipole antenna for ultra wideband communications using finite difference time domain method" (2003). FIU Electronic Theses and Dissertations. 2778.

https://digitalcommons.fiu.edu/etd/2778 
FLORIDA INTERNATIONAL UNIVERSITY

Miami, Florida

\begin{abstract}
MODIFIED DIAMOND DIPOLE ANTENNA FOR
ULTRA WIDEBAND COMMUNICATIONS USING

FINITE DIFFERENCE TIME DOMAIN METHOD
\end{abstract}

\author{
A thesis submitted in partial fulfillment of the \\ requirements for the degree of \\ MASTER OF SCIENCE \\ in
}

ELECTRICAL ENGINEERING

by

Srinivas Desamsetty

2003 
To: Dean Vish Prasad

College of Engineering

This thesis, written by Srinivas Desamsetty, and entitled Modified Diamond Dipole Antenna for Ultra Wideband Communications using Finite Difference Time Domain Method, having been approved in respect to style and intellectual content, is referred to you for judgment.

We have read this thesis and recommend that it be approved.

Subbarao V. Wunnava

Malcolm Heimer

Tadeusz M. Babij, Major Professor

Date of Defense: October 15, 2003

The thesis of Srinivas Desamsetty is approved.

Dean Vish Prasad College of Engineering

Dean Douglas Wartzok University Graduate School

Florida International University, 2003 


\section{DEDICATION}

With utmost respect and devotion, I sincerely dedicate this thesis to my parents and my spiritual guru, Sri Sri Sri Guru Viswa Spoorthi without Whose graceful help I would not have existed today. 


\section{ACKNOWLEDGMENTS}

I very earnest thank my advisor Dr. Tadeusz M. Babij without whose help this thesis could not have been accomplished. I wish to thank my committee members Dr. Subbarao V. Wunnava and Dr. Malcolm Heimer for their valuable suggestions and comments during the entire period of thesis work. I thank Pat Bammer and Carmen for their kind help and support. I wish to thank all my friends without whose help and encouragement my M.S would have been a very difficult task to achieve. I thank all the professors who extended their help in times of need through out my course work. I whole heartedly thank my Mother India for helping me build a strong foundation in my academics without which I would not have become eligible for acquiring my M.S. I would like to convey my thanks to U.S Government for giving me the opportunity to study in their Home Land.

I whole heartedly thank Dr. Subbarao V. Wunnava for his help, guidance and valuable support throughout my M.S. I once again express sincere gratitude towards my advisor Dr. Tadeusz M. Babij for showing me a better me in myself. 


\section{ABSTRACT OF THE THESIS \\ MODIFIED DIAMOND DIPOLE ANTENNA FOR ULTRA WIDEBAND \\ COMMUNICATIONS USING FINITE DIFFERENCE TIME DOMAIN METHOD \\ by}

Srinivas Desamsetty

Florida International University, 2003

Miami, Florida

Professor Tadeusz M. Babij, Major Professor

The aim of this thesis is to analyze and design ultra wideband antennas for personal communication devices using Finite Difference Time Domain (FDTD) method. Initially diamond dipole, an ultra wideband antenna is selected from the literature and analyzed. The shape of the diamond dipole is modified in such a way that the straight-line base of the two triangular arms (near the feed) is replaced with an arc. The resulting antenna is named as modified diamond dipole. Performance is observed by varying radius of curvature of base of triangular arms (for constant flare angle) and by varying flare angle (for constant radius of curvature of base of triangular arms). It is shown that modified diamond dipole offers better performance in terms of impedance bandwidth and return loss, than the diamond dipole with zero flare angle.

Slot loading technique has been applied to modified diamond dipole. It is shown that the resonant frequency of the slot loaded modified diamond dipole is decreased with increase in slot size and/or decrease in distance of the slot from the feed. 


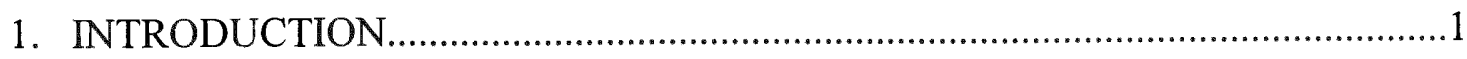

2. MICROSTRIP ANTENNAS FOR PERSONAL COMMUNICATION

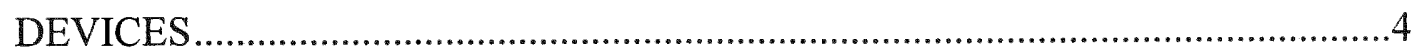

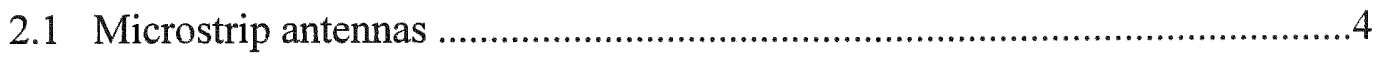

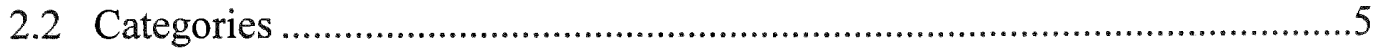

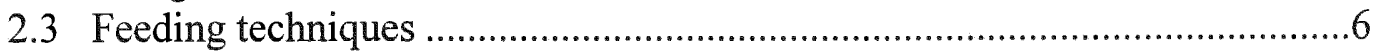

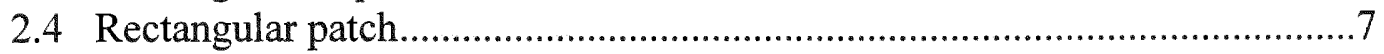

2.5 Figures of merit ..................................................................................

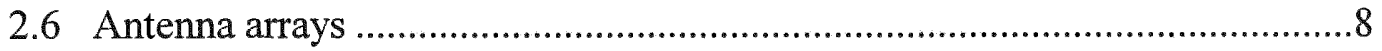

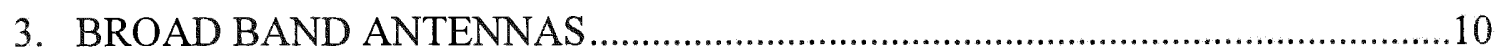

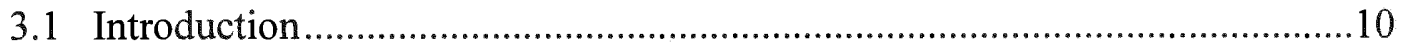

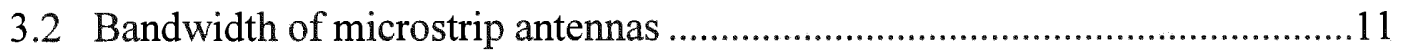

3.3 Bandwidth enhancement techniques .........................................................11

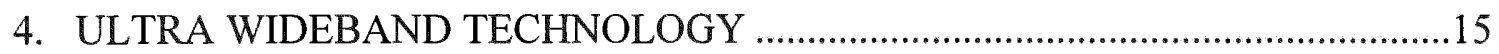

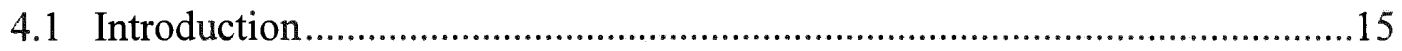

4.2 Intricacies of ultra wideband technology ..................................................... 18

4.3 Advantages of ultra wideband technology .................................................19

5. FINITE DIFFERENCE TIME DOMAIN (FDTD) METHOD ...............................21

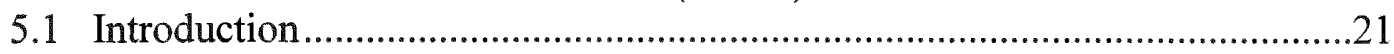

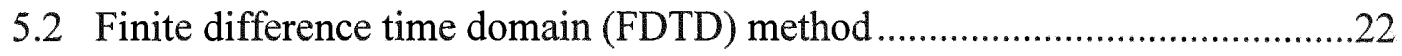

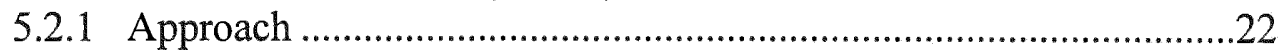

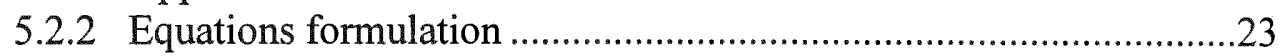

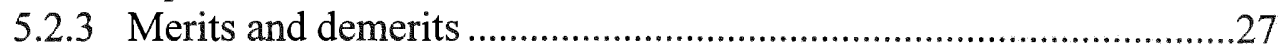

5.2.4 System requirements for XFDTD software ..................................29

5.2.5 Reason for choosing FDTD method .............................................29

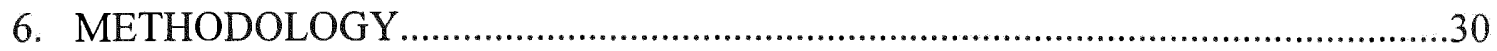

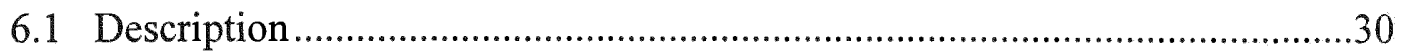

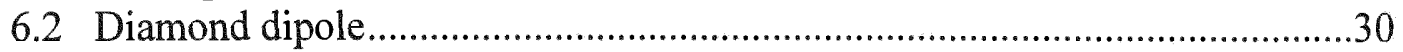

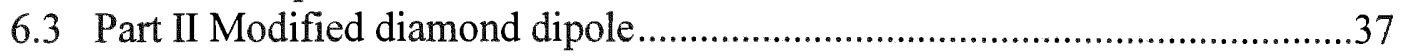

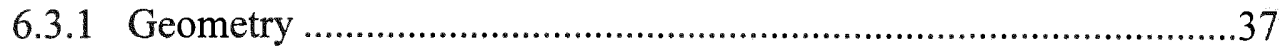

6.3.2 Modified diamond dipole, model MDIDO-A1 ...............................41

6.3.3 Modified diamond dipole, model MDIDO-B1 ..............................43

6.3.4 Modified diamond dipole, model MDIDO-C1 ...............................45

6.3.5 Modified diamond dipole, model MDIDO-A2 …............................47

6.3.6 Modified diamond dipole, model MDIDO-B2 …...........................49

6.3.7 Modified diamond dipole, model MDIDO-C2 …...........................51 
6.3.8 Modified diamond dipole, model MDIDO-A3 …...........................53

6.3.9 Modified diamond dipole, model MDIDO-B3 ...............................55

6.3.10 Modified diamond dipole, model MDIDO-C3 ...............................57

6.4 Part III Slot loaded modified diamond dipole .............................................5

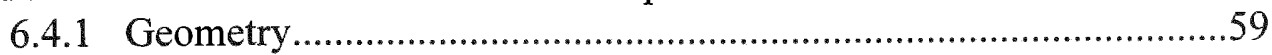

6.4.2 Slot loaded modified diamond dipole, model SLMDIDO-A...........60

6.4.3 Slot loaded modified diamond dipole, model SLMDIDO-B ...........62

6.4.4 Slot loaded modified diamond dipole, model SLMDIDO-C...........63

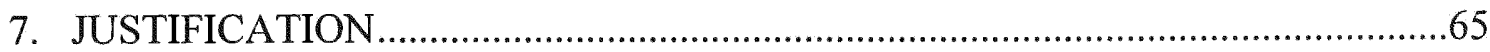

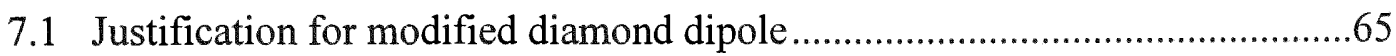

7.2 Justification for slot loaded modified diamond dipole .................................70

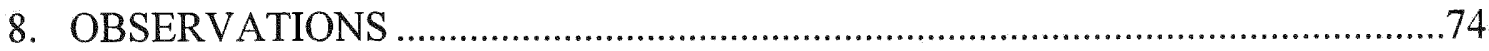

8.1 Observations made from various plots of modified diamond dipole ................................................................................

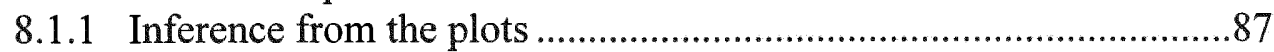

8.2 Observations made from various plots of slot loaded modified diamond dipole ............................................................................90

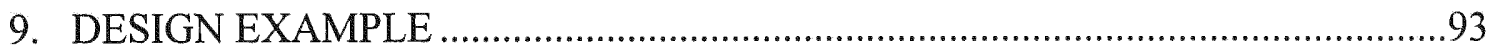

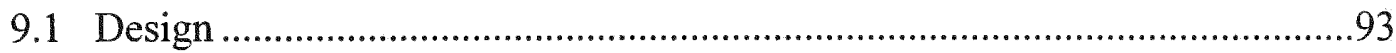

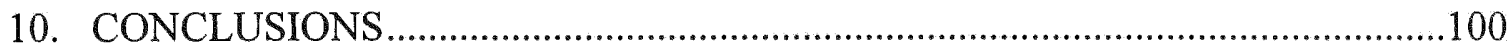

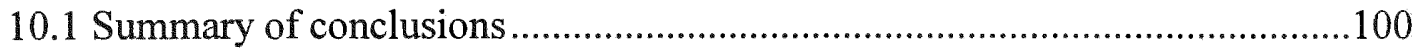

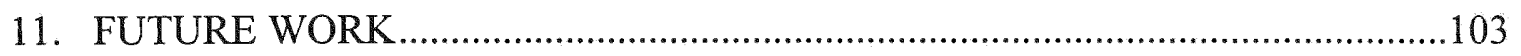

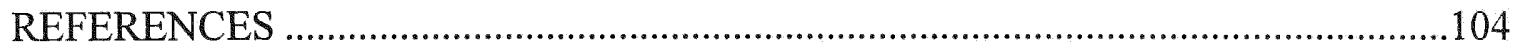




\section{LIST OF TABLES}

TABLE

PAGE

1. Information about the modified diamond dipole antenna models 40

2. Information about the slot loaded modified diamond dipole antenna models

3. Information about the antenna models selected for justification I .66

4. Information about the antenna models selected for justification II .71

5. Information about the modified diamond dipole antenna models .74

6. Resonant frequencies of MDIDO-A series antennas . .75

7. Resonant frequencies of MDIDO-B series antennas .77

8. Resonant frequencies of MDIDO-C series antennas .79

9. Resonant frequencies of MDIDO-A1, MDIDO-B1, MDIDOC1 81

10. Resonant frequencies of MDIDO-A2, MDIDO-B2, MDIDOC2 .83

11. Resonant frequencies of MDIDO-A3, MDIDO-B3, MDIDO-C3 .85

12. Information about the slot loaded modified diamond dipole antenna models.......90

13. Information about the most efficient antenna models in terms of return loss .94 


\section{LIST OF FIGURES}

FIGURE

1. Top view of an arbitrary shaped microstrip antenna........................5

2. Side view of the arbitrary shaped microstrip antenna ....................................... 5

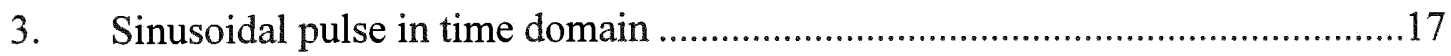

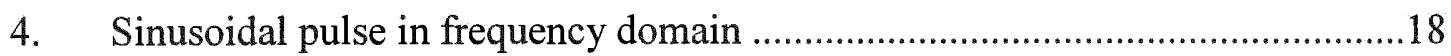

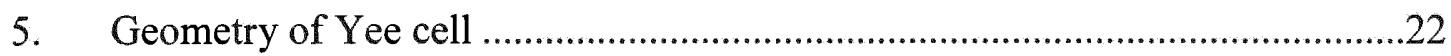

6. Diamond dipole antenna with dimensions .........................................................

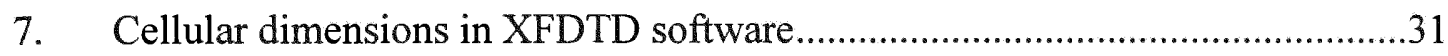

8. Cellular dimensions of diamond dipole antenna .............................................32

9. Layer $\mathrm{z}=33$ (xy plane) of diamond dipole in XFDTD software .........................33

10. Layer $\mathrm{x}=67$ (yz plane) of diamond dipole in XFDTD software........................33

11. 3-Dimensional view of diamond dipole in XFDTD software .............................34

12. Boundary condition selection in XFDTD software ............................................

13. Details of the modulated Gaussian pulse in XFDTD software ...........................35

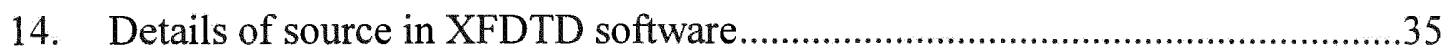

15. Time domain representation of modulated Gaussian pulse used as a source......36

16. Frequency Vs. return loss for diamond dipole antenna....................................36

17. Frequency Vs. input impedance for diamond dipole antenna .............................37

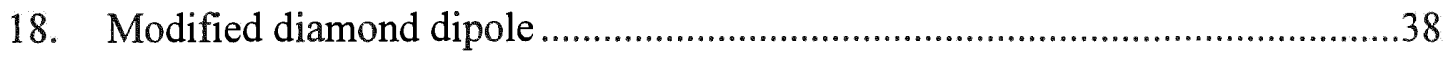

19. Cellular dimensions of modified diamond dipole antenna................................39

20. Radius of curvature alteration of modified diamond dipole..............................39

21. Flare angle alteration of modified diamond dipole ......................................40 
22. Layer $\mathrm{z}=33$ (xy plane) of MDIDO-A1 in XFDTD software

23. 3-Dimensional view of MDIDO-A1 in XFDTD software 41

24. Frequency Vs. return loss for MDIDO-A1 .42

25. Frequency Vs. input impedance for MDIDO-A1 .42

26. Layer $\mathrm{z}=33$ (xy plane) of MDIDO-B1 in XFDTD software .43

27. 3-Dimensional view of MDIDO-B1 in XFDTD software .43

28. Frequency Vs. return loss for MDIDO-B1 .44

29. Frequency Vs. input impedance for MDIDO-B1 44

30. Layer $\mathrm{z}=33$ (xy plane) of MDIDO-C1 in XFDTD software 45

31. 3-Dimensional view of MDIDO-C1 in XFDTD software .45

32. Frequency Vs. return loss for MDIDO-C1 46

33. Frequency Vs. input impedance for MDIDO-C1 46

34. Layer $\mathrm{z}=33$ (xy plane) of MDIDO-A2 in XFDTD software 47

35. 3-Dimensional view of MDIDO-A2 in XFDTD software 47

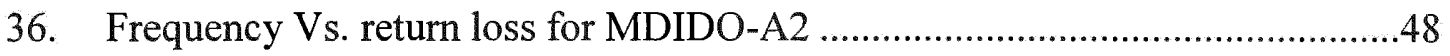

37. Frequency Vs. input impedance for MDIDO-A2 2...........................................48

38. Layer $z=33$ (xy plane) of MDIDO-B2 in XFDTD software ..........................49

39. 3-Dimensional view of MDIDO-B2 in XFDTD software …..........................49

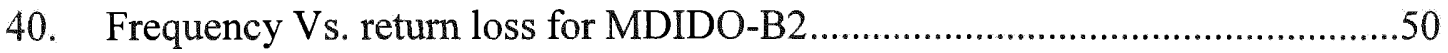

41. Frequency Vs. input impedance for MDIDO-B2 ..........................................50

42. Layer $\mathrm{z}=33$ (xy plane) of MDIDO-C2 in XFDTD software ...........................51

43. 3-Dimensional view of MDIDO-C2 in XFDTD software .................................51

44. Frequency Vs. return loss for MDIDO-C2 …...............................................52 


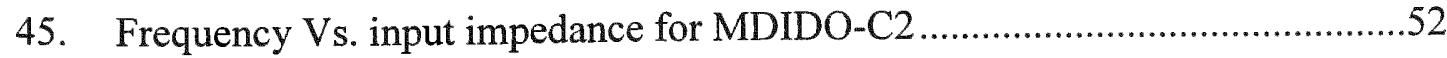

46. Layer $\mathrm{z}=33$ (xy plane) of MDIDO-A3 in XFDTD software …........................53

47. 3-Dimensional view of MDIDO-A3 in XFDTD software ................................53

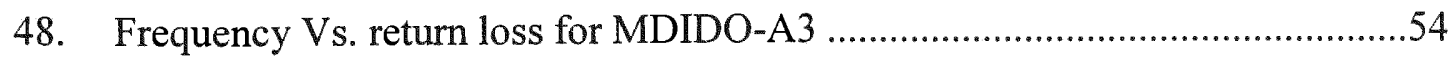

49. Frequency Vs. input impedance for MDIDO-A3 ............................................5

50. Layer $\mathrm{z}=33$ (xy plane) of MDIDO-B3 in XFDTD software ...........................55

51. 3-Dimensional view of MDIDO-B3 in XFDTD software ................................55

52. Frequency Vs. return loss for MDIDO-B3 .....................................................56

53. Frequency Vs. input impedance for MDIDO-B3 ............................................56

54. Layer $z=33$ (xy plane) of MDIDO-C3 in XFDTD software ............................57

55. 3-Dimensional view of MDIDO-C3 in XFDTD software ..................................57

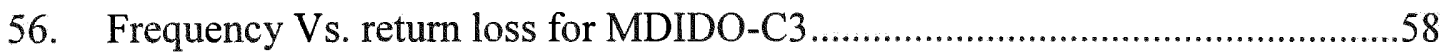

57. Frequency Vs. input impedance for MDIDO-C3 ….........................................5

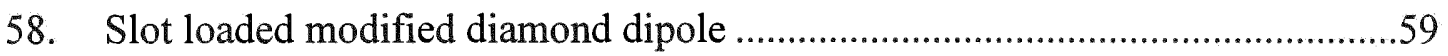

59. Layer $\mathrm{z}=33$ (xy plane) of SLMDIDO-A in XFDTD software ..........................60

60. 3-Dimensional view of SLMDIDO-A in XFDTD software .............................61

61. Frequency Vs. return loss for SLMDIDO-A .................................................61

62. Layer $\mathrm{z}=33$ (xy plane) of SLMDIDO-B in XFDTD software ..........................62

63. 3-Dimensional view of SLMDIDO-B in XFDTD software..............................62

64. Frequency Vs. return loss for SLMDIDO-B ...............................................63

65. Layer $\mathrm{z}=33$ (xy plane) of SLMDIDO-C in XFDTD software ........................63

66. 3-Dimensional view of SLMDIDO-C in XFDTD software.............................64

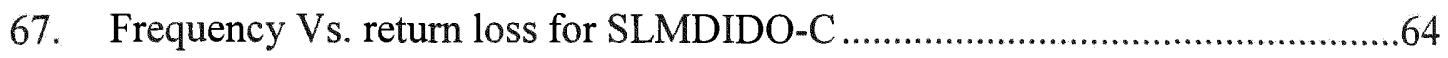


68. Justification model-I for justifying modified diamond dipole behavior

69. Justification model-II for justifying modified diamond dipole behavior

70. Resonant circuits for justification model-II

71. Coordinate where current density is measured .............................................68

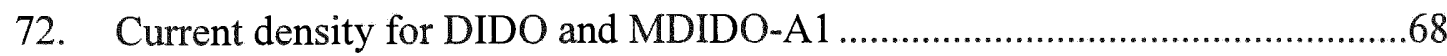

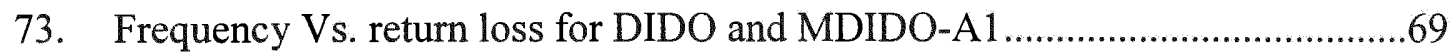

74. Frequency Vs. input resistance for DIDO and MDIDO-A1 ...........................69

75. Frequency Vs. input reactance for DIDO and MDIDO-A1 ...........................70

76. Current distribution in slot loaded modified diamond dipole ............................70

77. Equivalent circuit of modified diamond dipole .............................................71

78. Equivalent circuit of slot loaded modified diamond dipole .............................71

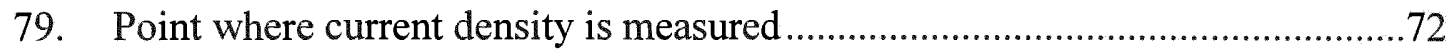

80. Current densities for MDIDO-A1 and SLMDIDO-A …...................................

81. Frequency Vs. return loss for MDIDO-A1 and SLMDIDO-A …......................73

82. Frequency Vs. return loss for MDIDO-A1, MDIDO-A2, MDIDO-A3 _.............75

83. Frequency Vs. input resistance for MDIDO-A1, MDIDO-A2, MDIDO-A3 .....76

84. Frequency Vs. input reactance for MDIDO-A1, MDIDO-A2, MDIDO-A3 .....76

85. Frequency Vs. return loss for MDIDO-B1, MDIDO-B2, MDIDO-B3 _............77

86. Frequency Vs. input resistance for MDIDO-B1, MDIDO-B2, MDIDO-B3 .....78

87. Frequency Vs. input reactance for MDIDO-B1, MDIDO-B2, MDIDO-B3 ......78

88. Frequency Vs. return loss for MDIDO-C1, MDIDO-C2, MDIDO-C3 ….........79

89. Frequency Vs. input resistance for MDIDO-C1, MDIDO-C2, MDIDO-C3 _....80

90. Frequency Vs. input reactance for MDIDO-C1, MDIDO-C2, MDIDO-C3 ......80 
91. Frequency Vs. return loss for MDIDO-A1, MDIDO-B1, MDIDO-C1

92. Frequency Vs. input resistance for MDIDO-A1, MDIDO-B1, MDIDO-C1 .....82

93. Frequency Vs. input reactance for MDIDO-A1, MDIDO-B1, MDIDO-C1 ......82

94. Frequency Vs. return loss for MDIDO-A2, MDIDO-B2, MDIDO-C2 ….........83

95. Frequency Vs. input resistance for MDIDO-A2, MDIDO-B2, MDIDO-C2 .....84

96. Frequency Vs. input reactance for MDIDO-A2, MDIDO-B2, MDIDO-C2 ......84

97. Frequency Vs. return loss for MDIDO-A3, MDIDO-B3, MDIDO-C3 ...........85

98. Frequency Vs. input resistance for MDIDO-A3, MDIDO-B3, MDIDO-C3 .....86

99. Frequency Vs. input reactance for MDIDO-A3, MDIDO-B3, MDIDO-C3 ......86

100. Current density for MDIDO-A1, MDIDO-A2, MDIDO-A3 …......................87

101. Current density for MDIDO-B1, MDIDO-B2, MDIDO-B3 …..........................8

102. Current density for MDIDO-C1, MDIDO-C2, MDIDO-C3 …........................8

103. Figure showing how arc length increases with flare angle ..............................89

104. Slot loaded modified diamond dipole with regions D1 and D2 _.....................91

105. Frequency Vs. return loss of slot loaded modified diamond dipole models ......91

106. Current density of various SLMDIDO models ..............................................92

107. Modified diamond dipole with base having radius of curvature equal to the radius of the incircle of triangular arm of diamond dipole ..............................93

108. Comparison of frequency Vs. return loss for various antenna models ..............95

109. Modified diamond dipole to circular dipole transformation ..............................95

110. Frequency Vs. return loss of a circular dipole of radius $\mathrm{R}=19 \mathrm{~mm} \ldots \ldots \ldots \ldots \ldots . . . . . . .66$

111. Frequency Vs. return loss for most efficient modified diamond dipole.............97

112. Comparison of gain for modified diamond dipole and diamond dipole at $1.8 \mathrm{GHz}$ 
113. Comparison of gain for modified diamond dipole and diamond dipole at $3.6 \mathrm{GHz}$.

114. Comparison of gain for modified diamond dipole and diamond dipole at $5.9 \mathrm{GHz}$.

115. Comparison of gain for modified diamond dipole and diamond dipole at $7.3 \mathrm{GHz}$. 


\section{CHAPTER 1}

\section{INTRODUCTION}

We are living in an information age. There are two ways of communicating a message to distant places. Through wired connection and wireless connection. The world is moving more and more towards the wireless connection i.e. wireless communication.

Guglielmo Marconi was the first to see the possibility of wireless communication when he demonstrated radio's ability to provide continuous contact with ships sailing the English Channel [1]. From then new wireless communications methods and services have been adapted enthusiastically. With the advent of digital technology and RF fabrication techniques, mobile radio communications have grown in large number. With the advent of the miniaturizing technologies, radio communication equipment has changed from small to portable and from portable towards more and more miniature in size. Digital switching techniques have facilitated the large deployment of affordable, easy to use radio communication networks. The number of cellular telephone users grew from 25,000 in 1984 to about 16 million in 1994, and since then, wireless services have been experiencing customer growth rates well in excess of $50 \%$ per year [1].

Though the customers for wireless communications are increasing continuously, available bandwidth is always a constant. Hence many new wireless technologies like the FDMA, TDMA, CDMA, SDMA etc. which split the frequency spectrum according to their own criterion have evolved. These developments have laid more and more constraints in the hardware design issues of the communication systems, like the antenna bandwidth, filter design, amplifier design etc. Almost everyday a new service is being 
added to the existing services of wireless communications. This ranges from very low bandwidth short message services to very high bandwidth videoconference [2]. Hence, with such high data rate requirements (with constant and scarce bandwidth), it seems one day in the future, the bandwidth will be inadequate. Hence, new technologies in wireless communications have to be evolved day by day to accommodate the ever growing users of wireless communications.

Until recently (and even today), in many areas, continuous sinusoidal waves and semi-sinusoidal waves are used for communication purpose. It is estimated that ultra wideband technology brings a new trend in wireless communications. Ultra wideband (UWB) technology is a technology- which uses short pulses rather than semi-continuous sinusoidal waveforms for communication, thereby transmitting the signal energy over a wide frequency band. Ultra wideband technology needs no carrier to carry the information. This new technology can directly modulate an impulse, hence can be called as carrierless technology. FCC has defined ultra wideband systems as those having a bandwidth more than $20 \%$ of the center frequency or more than $500 \mathrm{MHz}$, which ever is smaller [3]. Bandwidth efficiency, defined as throughput data rate per hertz is a very important parameter to be considered. It provides a measure of how efficiently the bandwidth is utilized. From the definition of ultra wideband, stated above it can be understood that this technology has a very good bandwidth efficiency. Shannon's channel coding theorem states that for an arbitrarily small probability of error, the maximum possible bandwidth efficiency is limited by the noise in the channel [1].

In case of ultra wideband technology which uses very short pulses for transmission, millions of pulses are transmitted in very short time. However, the problem 
lies in designing a receiver and generator with exception timing. Since the pulses carry very low power, the signal energy is distributed over a very large bandwidth. Some of the advantages of ultra wideband technology may be summarized as low power, low cost, high data rates, precise positioning capability and no interference. Multipath distortion is eliminated in UWB systems as the delayed copy which arrives outside the timing window (time during which the receiver is active) is heard as noise and will be rejected. As the technology utilizes pulses with very low power than ordinary transmitters, it is very attractive to use in portable devices and mobile communication systems [2].

There is a lot of ongoing research to design new transmitters, receivers and antennas for ultra wideband communications. Antenna is one of the most difficult parts of a communication system to design because much of the literature developed for sinusoidal wave transmission is of no use. Hence, much work has been devoted in the design of ultra wideband antennas [4]-[10]. Ordinary wideband antennas will generally not transmit fast transients because they have not been corrected for dispersion. This thesis focuses on the design of ultra wideband microstrip antennas, keeping in mind the bandwidth and miniaturizing requirements of today's technology. 


\section{CHAPTER 2}

\section{MICROSTRIP ANTENNAS FOR PERSONAL COMMUNICATION DEVICES}

In many applications like personal communication devises and satellite communications, weight and space occupied by the communication system is given great importance. Hence, miniaturizing the communication system components and hence, the antenna has become a necessity and challenging factor for many years. Deschamps first proposed the concept of microstrip radiators in 1953 and Howell and Munson developed the first practical antenna [11]. Much work has been devoted in the past for this purpose [12]-[16]. This chapter provides information about the concept of microstrip antennas, different types and design issues.

\subsection{Microstrip antennas}

As shown in Fig. 1 and Fig. 2, a microstrip antenna consists of a dielectric substrate sandwiched by radiating patch and a ground plane on either side. The patch (normally copper or gold) can assume virtually any shape, but shapes like rectangle, square, circle, ring etc. are used to simplify the analysis and performance prediction [11]. Because of advantages like light weight, low volume, low fabrication cost, thin profile configuration, linear and circular polarizations made possible with simple feed, easily integrable with microwave integrated circuits, readily amenable to mass production, etc microstip antennas became popular inspite of the disadvantages like narrow bandwidth, lower gain and efficiency, difficulty in achieve polarization purity, excitation of surface waves, extraneous radiation from feeds and junctions, etc. However there are methods to reduce most of the limitations of microstrip antennas, and use the advantages they offer. A patch radiates efficiently when it is resonant, which means that some characteristic 
dimension of the patch is nearly equal to one half wavelength in the substrate medium [2].

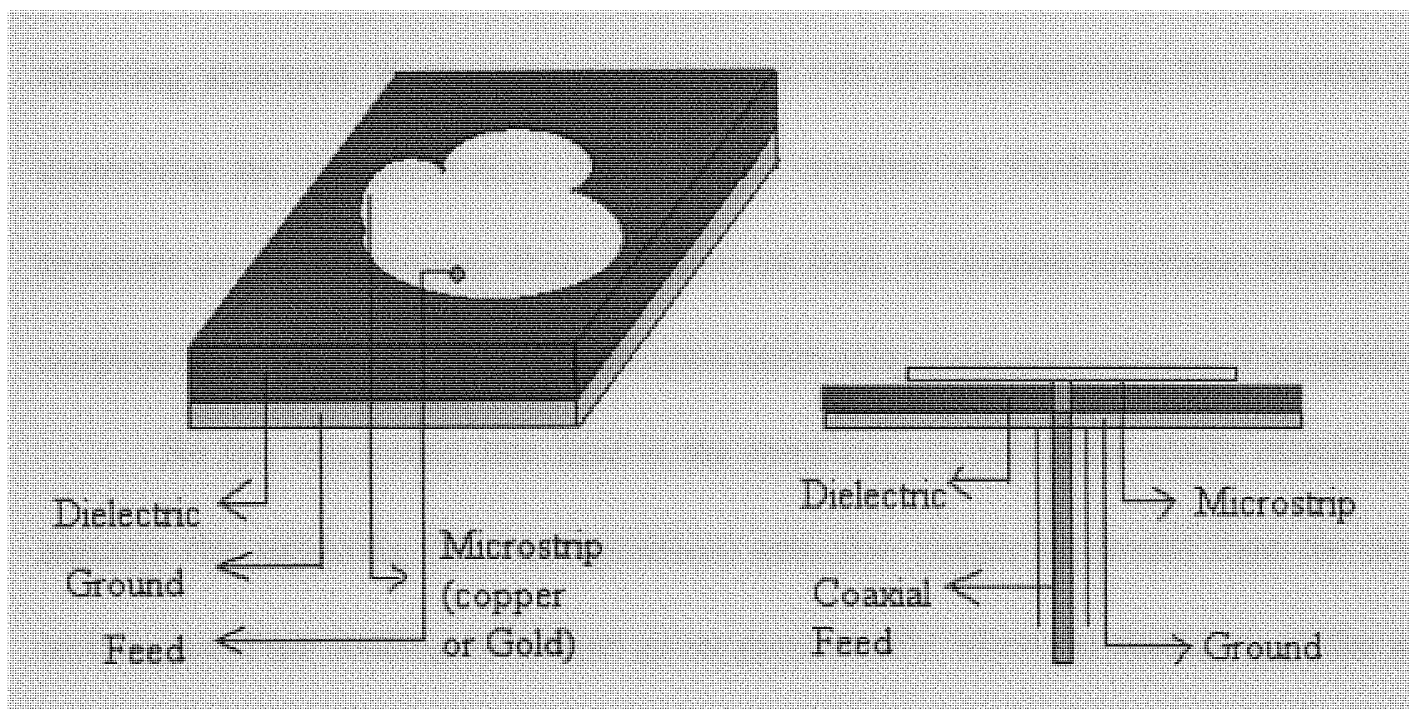

Fig. 1. Top view of an arbitrary

Fig. 2. Side view of the arbitrary

shaped microstrip antenna. shaped microstrip antenna.

\subsection{Categories}

All microstrip antennas can be divided into four basic categories [11]

- Microstrip patch antennas

- Microstrip dipoles

- Printed slot antennas and

- Microstrip traveling wave antennas.

Various techniques have been explored in analyzing microstrip patch antennas, including transmission line models, cavity models, full-modal expansions, and various numerical techniques. Transmission-line model is the easiest of all but gives inaccurate results and lacks versatility where as the cavity model though difficult, provides more accuracy. 
Cavity model is similar to perturbation methods [17]. Recently, finite difference time domain (FDTD) method has emerged as a numerical technique of choice.

\subsection{Feeding techniques}

The purpose of a transmission line is to transfer the power efficiently from the feed to the radiating structure. Feed plays an important role in miniaturizing the antenna size and improving its characteristics. There are a number of feeding techniques developed, however prominent among them are coaxial feed, microstrip (coplanar feed), proximity coupled microstrip feed, aperture coupled microstrip feed, and coplanar waveguide feed.

So far, microstrip line feed is the most easy to fabricate, easily matchable to the antenna (by adjusting the inset position) and also simple to model than the remaining techniques. This feeding technique gives good results when the thickness of the substrate is very small. However, surface waves and spurious radiation increases with substrate thickness and limits the bandwidth [17]. Coaxial feed is also easy to fabricate, but has less bandwidth. However, it is difficult to model for thick substrates. The interesting feature is that it has very less spurious radiation. Though, proximity coupled microstrip feed is difficult to construct, it has the largest bandwidth of all the methods mentioned above, easy to model and has low spurious radiation [17].

Feed plays an important roll in increasing the bandwidth of an antenna. Usually, designing an antenna involves the design of the feed network also. In [18] it has been reported that by using capacitively coupled feed, overall length of a shorted patch antenna with an air substrate can be reduced from one-quarter wavelength to less than one-eigth wavelength. In [18], it has been shown that an impedance bandwidth of $39 \%$ can be obtained for an L-probe fed shorted patch antenna. 


\subsection{Rectangular patch}

It is stated that symmetrical structures are easier to analyze [17]. Rectangular patch being the most basic symmetric structure is taken as an example for a brief discussion. Considering transmission line model, microstrip antenna is seen as two slots separated by low impedance transmission line. As the patch dimensions are finite, fields undergo fringing at both the slots. Fringing is a function of the dimensions of the patch. But for most microstrip antennas Length is very much greater than its height $(\mathrm{L} / \mathrm{h}<<1)$ fringing is reduced to a great extent. But even the least fringing effect has to be taken into account as it effects the resonant frequency. The electric lines concentrate mostly in the dielectric medium. But since, they travel through two different media (air and substrate) an effective dielectric constant has to be found to find the correction factor introduced by fringing. Effective dielectric constant $\left(\varepsilon_{\text {reff }}\right)$ is defined as the dielectric constant of the uniform dielectric material, which produces the same effect, produced by the combinations of the dielectric materials. Fringing causes the antenna to appear larger in dimensions that it is. In [17] the design procedure for a rectangular patch is given as follows. When relative dielectric constant of substrate $\left(\varepsilon_{\mathrm{r}}\right)$, resonant frequency $\mathrm{f}_{\mathrm{r}}$ (in hertz) and height (h) (in meters)are specified, width (W) and length (L) are determined as follows.

$$
\begin{aligned}
& W=\frac{1}{2 f_{r} \sqrt{\mu_{0} \varepsilon_{0}}} \sqrt{\frac{2}{\varepsilon_{r}+1}}=\frac{v_{0}}{2 f_{r}} \sqrt{\frac{2}{\varepsilon_{r}+1}} \\
& v_{0}=\text { velocity of light in air. } \\
& \varepsilon_{\text {reff }}=\frac{\varepsilon_{r}+1}{2}+\frac{\varepsilon_{r}-1}{2}\left[1+12 \frac{h}{W}\right]^{-\frac{1}{2}}
\end{aligned}
$$




$$
\frac{\Delta L}{h}=0.412 \frac{\left(\varepsilon_{\text {reff }}+0.3\right)\left(\frac{W}{h}+0.264\right)}{\left(\varepsilon_{\text {reff }}-0.258\right)\left(\frac{W}{h}+0.8\right)}
$$

$\Delta L=$ Extension of length

$$
L=\frac{1}{2 f_{r} \sqrt{\varepsilon_{r e f f}} \sqrt{\mu_{0} \varepsilon_{0}}}-2 \Delta L
$$

\subsection{Figures of merit}

For any antenna, quality factor $(\mathrm{Q})$, bandwidth, and efficiency are the figures of merit. These are interrelated and there is no complete freedom to independently optimize each one [17]. Quality factor depends on the losses of the antenna. The reciprocal of total quality factor is defined as the sum of the reciprocals of quality factors due to individual losses of the antenna. Since some of the losses depend on the physical dimensions of the antenna, it can be said that the quality factor depends on the physical dimensions of the antenna.

\subsection{Antenna arrays}

In most cases the characteristics like the gain, directivity or beamwidth etc. of a single antenna cannot fulfill the requirement. This situation can be over come by using many antenna elements in a particular pattern, called an antenna array. The pattern might be in a linear, a planar or a volumetric fashion and hence the names linear array, planar array, volume array. The required application dictates the type of array to be designed. Usually, all elements (antennas) of an array are identical (though not a necessity). Selecting identical elements, only helps to design the antenna array easily. 
Overall pattern of an array depends on the geometrical configuration of the array (linear, planar or volume), relative displacement of array elements, excitation amplitude, excitation phase and relative pattern of the individual elements. In general, radiation characteristics of an array can be determined once the aperture distribution is known. The amplitude and phase distribution of the feed is usually determined according to the intended application. The means of excitation of the radiating elements is thus an essential and important factor. The feeds are categorized as parallel and series feeds (as per their geometries) [11].

By properly selecting the above-mentioned factors, antenna array of desired characteristics can be designed. Now a days, algorithms are being incorporated, which enable an antenna array to steer the beam towards the users. 


\section{CHAPTER 3}

\section{BROADBAND ANTENNAS}

\subsection{Introduction}

The antenna bandwidth can be measured by using standing wave ratio (SWR) parameter. Bandwidth can be defined as range of frequencies over which the SWR is below a certain predefined mark (usually 1.5 to 2 , and in some cases 3 ). Antennas, which have a good impedance match over a wide frequency range, are called broadband antennas. It has been reported in [17] that quality factor, bandwidth and radiation efficiency is interrelated and in order to achiever the efficiency over one parameter, efficiency over other parameter have to be sacrificed. The fractional bandwidth of the antenna is inversely proportional to the total quality factor of the antenna [17].

$$
B W=\frac{2(k r)^{3}}{\eta}
$$

$\mathrm{BW}=$ band width, $\eta=$ efficiency

where $r=\frac{\lambda}{2 \pi}$ and $\mathrm{k}$ is wave number [22] where $\lambda=$ wavelength .

The above expression [22] states that, if the antenna size is constrained, instantaneous bandwidth (BW) is gained by sacrificing some of the radiation efficiency. The standing wave ratio bandwidth can be expressed in terms of percentage bandwidth using (3.2)

SWR bandwidth $=\frac{\left(f_{2}-f_{1}\right)}{f_{c}} \times 100 \%$

$f_{1}$ and $f_{2}$ are the lower and upper frequencies respectively, over which the SWR is less than a predefined value. $f_{c}$ is the center frequency, given by 
$f_{c}=\sqrt{f_{1} \times f_{2}}$

Since microstrip antennas are inherently band limited, much of the research is devoted in increasing its bandwidth.

\subsection{Bandwidth of microstrip antennas}

Along with low radiation efficiency and high quality factor $(\mathrm{Q})$, very narrow frequency bandwidth is a major disadvantage and a challenging parameter of microstrip antennas which needs to be improved. Lot of work has been devoted in the past to achieve broadband operation of microstrip antennas.

\subsection{Bandwidth enhancement techniques}

Increasing substrate thickness increases bandwidth of an antenna. This is a very basic technique, but results in a low quality factor, Q. Hence, tradeoff between bandwidth and quality factor must be made before enhancing the antenna bandwidth by this method. Antenna bandwidth can also be increased by increasing the relative permittivity of the dielectric material used as substrate. Relative permittivity and thickness of the substrate may be selected properly to obtain a desirable bandwidth. Another technique is to design a wideband-matching network. Some of the other bandwidth enhancement techniques are mentioned below.

i) Stacked elements

It has been shown that a wider bandwidth can be achieved with microstrip antennas in stacked patch configuration [11]. A number of parameters like substrate thickness $d_{1}$ and $d_{2}$, dielectric constants $\varepsilon_{\mathrm{r} 1}$ and $\varepsilon_{\mathrm{r} 2}$, patch sizes, offset between the centers of the patches and feed location are to be considered to design an antenna with a desired bandwidth. For example, in case of antenna with two layers, the size of the upper patch is 
selected slightly smaller or larger than the lower patch to obtain a slightly different resonant frequency. Various other parameters are optimized to achieve broadband operation.

ii) Stacked shorted patches

It has been shown in [18] that by making two stacked shorted patches radiate as equally as possible and having a radiation quality factor as low as possible, enhanced impedance bandwidth can be obtained for a fixed antenna volume. If the two stacked shorted patches have different shorting walls, then selecting the distance between the two offset shorting walls is crucial for achieving large operational bandwidth. If the two stacked-shorted patches have a common shorting wall, then the feed location becomes crucial factor in achieving a wideband performance.

iii) Coplanar parasitic elements

A set of coplanar resonators with slightly different resonant frequencies can be configured to obtain broadband performance. The feed line drives only one patch, usually the center patch, and the other patches are either gap coupled or directly coupled to the driven patch. The idea behind selecting resonators with different lengths is that, four resonant modes can be excited at frequencies close to each other, thus obtaining a wide band performance. This concept can further be extended to gap coupled arrays [11]. iv) Multimode technique

In this approach, two independent modes are excited in the same patch or in the patch and the feeding network. In [19] an E-shaped microstrip antenna is developed such that the patch and the slot are designed to produce resonances close to each other, obtaining wideband impedance bandwidth. 
v) Impedance matching

By using an impedance matching network in the feed portion of microstrip antenna, impedance bandwidth can be improved. To obtain better results (bandwidth and efficiency), the distance between the matching network and radiating patch must be kept as small as possible. In an approach discussed in [11], the impedance matching network is embedded into the patch itself. The matching network is designed such that at resonance, it produces a reactive behavior opposite to that of the patch. The loaded patch will now have two resonances close to each other giving rise to broadband operation.

vi) Slot loading

By embedding suitable slots in a radiating patch, compact operation with an enhanced impedance bandwidth can be obtained. The impedance bandwidth obtained for such a design is usually equal to or less than 2.0 times that of the corresponding conventional microstrip antenna. To achieve a much greater impedance bandwidth, one can use compact designs with chip-resistor loading [18].

vii) Resistive loading

The impedance bandwidth of a patch antenna can be increased by introducing losses in antenna, but at the expense of decreased radiation efficiency [11]. A chip resistor of $1 \mathrm{ohm}$ can be incorporated for this purpose. The obtained impedance bandwidth can be increased by a factor of six compared to the design using shorting pins discussed in [2].

viii) Reactive loading

Inserting a microstrip line section at one of the radiating edges of a rectangular patch provides an integrated reactive loading to the microstrip antenna. By varying the 
dimensions of the inserted microstrip line section, two resonant modes near the fundamental mode of the original unloaded microstrip antenna can be excited, making dual frequency operation possible. Further, by applying this inserted loading technique and modifying the inserted microstrip-line section to an inserted microstrip structure of cascaded transmission line sections, the two resonant modes can be made to excite at frequencies very close to each other to form a wideband operation. This technique can be extended to square, circular and triangular patch shapes [18].

ix) Modified probe feed

For conventional probe-fed microstrip antennas, the large probe reactance owing to long probe pin is undesirable. In [18] it has been reported that, an annular ring slot or a narrow rectangular ring slot can be cut to solve this problem. By choosing suitable dimensions of the ring slot, the large probe reactance can be obtained. By using a capacitively coupled feed, or an L-probe/L-strip coupled feed, similar probe compensation can be obtained.

x) Varactor diode

A varactor diode can be used to obtain dual frequency operation. By careful design, one can obtain the two resonant frequencies very close to each other thereby achieving wideband operation. Two diodes are positioned symmetrically in the patch to minimize the cross polarization effects, and the relationship between the power and the bias voltage level of the varactor diode represents a way of tuning the structure [2]. Hence by using any of the above methods or a combination, one can obtain broadband performance of microstrip antennas, defeating their disadvantage of very low bandwidth. 


\section{CHAPTER 4}

\section{ULTRA WIDEBAND TECHNOLOGY}

\subsection{Introduction}

The concept of ultra wideband can be well understood if we consider the frequency domain and time domain representations of a pulse. For a better understanding Fourier Transform and Discrete Fourier Transform are discussed.

A signal can be viewed in time domain and frequency domain. Fourier Transform is a mathematical tool, which can be used to transform to convert the signal from time domain to frequency domain representation. The Fourier Transform of a waveform $w(t)$ is defined as

$W(f)=F[w(t)]=\int_{-\infty}^{\infty}[w(t)] e^{-j 2 \pi f t} d t$

$F[$.$] is the Fourier Transform of [.] and \mathrm{f}$ is the frequency parameter with units of $\mathrm{Hz} . \mathrm{F}$ is the frequency. With the availability of high speed computers and signal processors, the spectrum of a waveform is approximated using Discrete Fourier Transform (DFT). DFT is defined as

$$
X(n)=\sum_{k=0}^{k=N-1} x(k) e^{-j(2 \pi / N) n k} \quad \mathrm{n}=0,1,2 \ldots \mathrm{N}-1
$$

A program has been written in MATLAB to obtain the time domain and frequency domain of a sinusoidal pulse which is shown in the next page.

MATLAB Program for the generation of sinusoidal pulse in Time and Frequency Domain.

$\mathrm{M}=7$; 
$\mathrm{N}=2^{\wedge} \mathrm{M}$;

$\mathrm{n}=0: 1: \mathrm{N}-1$;

tend $=2$;

$\mathrm{T}=10$;

$\mathrm{dt}=\mathrm{T} / \mathrm{N}$

$\mathrm{t}=\mathrm{n} * \mathrm{dt}$

$\%$ creating time waveform

$\mathrm{w}=\mathrm{zeros}($ length $(\mathrm{t}), 1)$;

for $(\mathrm{i}=1: 1:$ length $(\mathrm{w}))$

if $(\mathrm{t}(\mathrm{i})<=$ tend $)$

$\mathrm{w}(\mathrm{i})=\sin \left(2^{*} \mathrm{pi}^{*} \mathrm{t}(\mathrm{i})\right)$;

else $w(i)=0$;

end;

end;

$\operatorname{plot}(\mathrm{t}, \mathrm{w})$

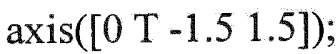

xlabel('t (sec) --->');

ylabel('w(t)');

title('Time Waveform');

pause;

figure;

$\mathrm{W}=\mathrm{fft}(\mathrm{w})$;

$\mathrm{f}=\mathrm{n} / \mathrm{T}$; 
$\mathrm{W}=\mathrm{abs}(\mathrm{W})$;

$\operatorname{plot}(\mathrm{f}, \mathrm{abs}(\mathrm{W}))$;

$\operatorname{axis}\left(\left[\begin{array}{llll}0 & 10 & 0 & 40\end{array}\right]\right)$

xlabel('f (Hz) --->');

ylabel('|W(f)|');

title('Frequency domain');

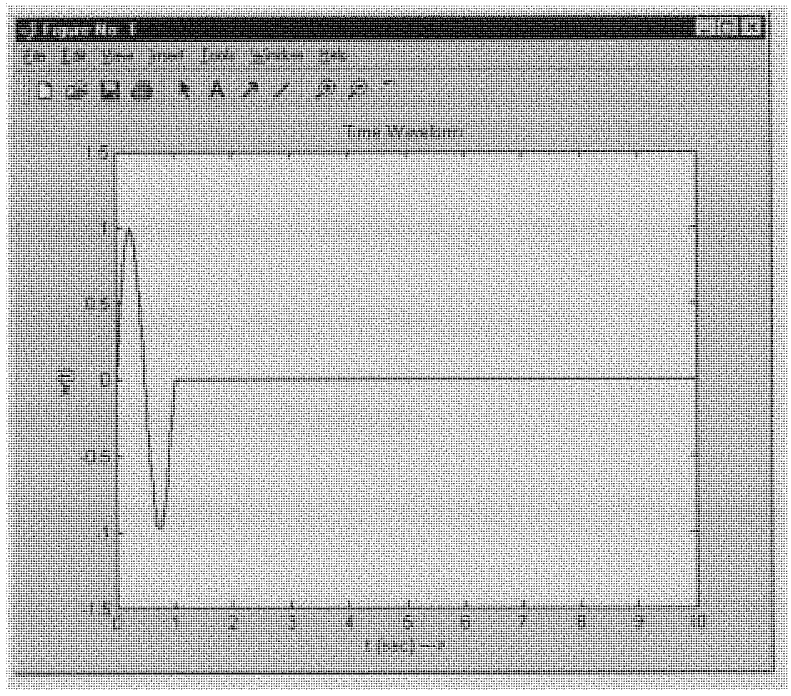

Fig. 3. Sinusoidal pulse in time domain.

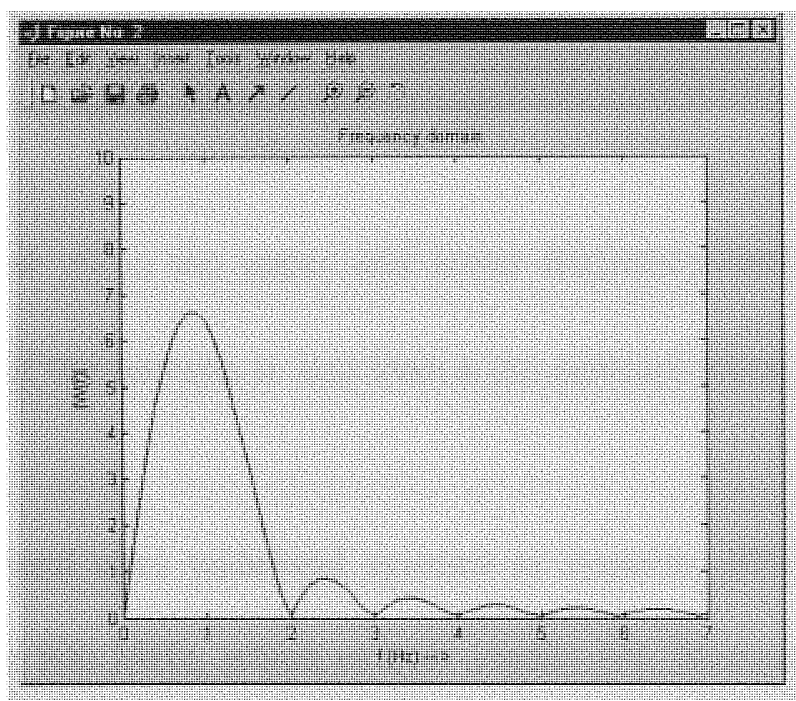

Fig. 4. Sinusoidal pulse in frequency domain. 
It can be seen that the waveform in frequency domain distributes the energy over a very wide frequency range. This technology of utilizing pulse for communication is called ultra wideband technology. Ultra wideband technology is a technology- which uses short pulses rather than semi-continuous sinusoidal waveforms for communication, thereby transmitting the signal energy over a wide frequency band. Using pulses is not a new technique. This technology has been used in ground penetration radar and much work has been done in that area. It is only recently that this technology has been considered for personal communications and many patents were reported. The most interesting part is that ultra wide band technology makes use of the garbage frequency, ISM (industrial, scientific, medical) band of frequencies.

\subsection{Intricacies of ultra wideband technology}

The basic idea behind ultra wideband is to transmit low power pulses (usually Gaussian) whose rise time is very small, typically ranges from few pico-seconds to hundreds of nano-seconds. The signal occupies a very wide spectrum of bandwidth. Percentage bandwidth $(\% \mathrm{BW})$ is an important parameter to be defined in this context.

$$
\% B W=\frac{2\left(f_{H}-f_{L}\right)}{f_{R}} \times 100
$$

where $f_{H}$ and $f_{L}$ is the upper and lower frequency limit between which the standing wave ratio of the antenna is less than a pre-selected value. $f_{R}$ is the frequency of resonance.

FCC has defined ultra wideband systems as systems, which have a percentage bandwidth of $20 \%$ or $500 \mathrm{MHz}$, which ever is smaller [3]. Because of high data rate in the form of pulses, non-ultra wideband receivers (designed for receiving continuous 
sinusoidal waves) sense these pulses as noise (as they could not understand pulses with high data rates). But, the challenging factor is the design of the system. The transmitter needs to be designed to transmit millions of pulses in very short time and the receiver to receive the pulses with exceptional timing. Another challenging factor is to design antenna, which have to radiate the fast transients (pulses) without much distortion, which is the topic of thesis.

\subsection{Advantages of ultra wideband technology}

Ultra wideband technology overcomes many of the limitations of narrowband technology.

- The straightforward advantage is the bandwidth it offers. Narrowband systems are characterized to have a fractional bandwidth of $10 \%$ or less of center frequency, where as ultra wideband systems are characterized to have a fractional bandwidth of $20 \%$ or more of center frequency [2], [3].

- Ultra wideband technology can transmit data from very high rates (for short range communications) to very low rates (for telemetry applications).

- Ultra wideband being a carrier less technology reduces the cost of architectures for communication systems.

- Fading is a very important limitation to be considered. Fading occurs when multipath components of the signal (signal components taking different paths by reflection, refraction and scattering) cause destructive interference (signals opposite in phase). Ultra wideband systems are more robust to fading and interference than narrowband systems. Ultra wideband devices can incorporate multiple parallel receivers, known as RAKE receiver architecture, to coherently 
add the energy from the many suitable reflected signals to increase the signal-tonoise ratio [2].

- Ultra wideband technology is utilized to detect objects to a very high precision (typically from tens of meters to a few centimeters).

- Because of low power requirements, the batteries of the personal communications devises can operate for a longer time.

The areas where the ultra wideband technology can be used are personal area networking, military warfare, motion tracking etc. There is a constant search for areas where the ultra wideband technology can be used. 


\section{CHAPTER 5}

\section{FINITE DIFFERENCE TIME DOMAIN (FDTD) METHOD}

\subsection{Introduction}

The starting point for any electromagnetic problem is the Maxwell's equations [20]

$$
\begin{aligned}
\nabla \times E & =-\frac{\partial B}{\partial t} \\
\nabla \times H & =\frac{\partial D}{\partial t}+J \\
\nabla . D & =\rho \\
\nabla . B & =0
\end{aligned}
$$

In case of any structure, the fields inside, its visualization, and the way it radiates is very difficult to find out. This is one of the reasons why, most antennas are symmetric structures. Symmetric structures can be analyzed with relative ease. Though expressions are developed by Kraus, Tai, Pozar and others for some structures they only give approximate results and a more accurate results might be desirable [17]. With the advent of high speed computers with memories of hundreds of giga bytes, numerical techniques became very effective tools than any other tool to study the behavior of any electromagnetic scatterer or radiator.

FDTD (finite difference time domain), MoM (method of moments) and FEM (finite elements method) are some of the most popularly employed numerical techniques for electromagnetic analysis. In this research only FDTD method is used as a analyzing tool. In the next section, a brief discussion of how the FDTD method works is outlined. 


\subsection{Finite difference time domain (FDTD) method}

Out of the many approaches (method of moments, finite element analysis, physical optics etc.) FDTD technique is the one, which can be applied to large number of electromagnetic problems (electromagnetic radiation, interaction, and scattering). FDTD method was developed by Kane S. Yee, in 1966 [20]. FDTD method is a time domain technique, which means the fields are computed as a function of time. Initially, this method did not gain much popularity because of the huge computational overhead. But, with the advent of high-speed computers with memories of the order of several hundreds of giga bytes, FDTD method became the most widely employed method. Computer science, computer engineering and computational engineering are the three fields applied to develop the FDTD method.

\subsubsection{Approach}

The basic building block of FDTD method is the Yee Cell, shown below.

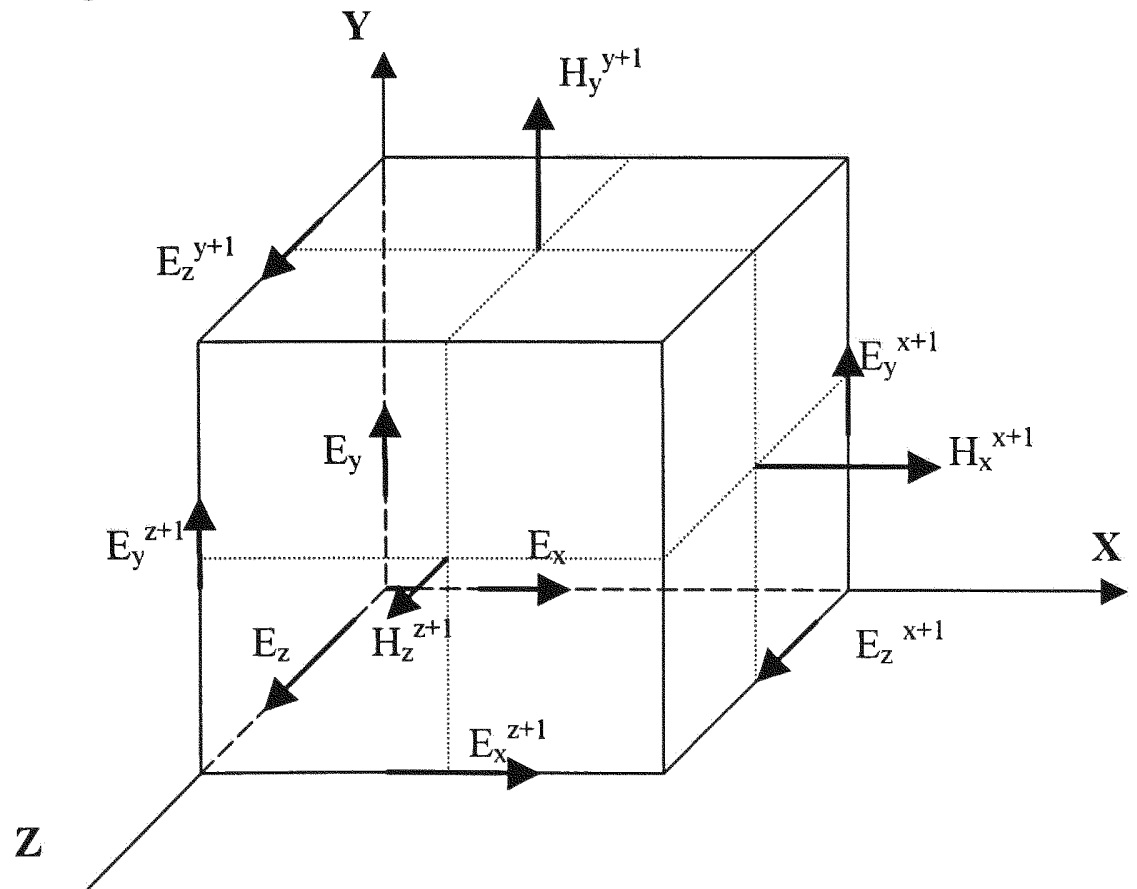

Fig. 5. Geometry of Yee cell. 
In Fig. $3, E_{x}, E_{y}, E_{z}$ are the $x, y$ and $z$ components of electric field at location $(x, y, z)$. $\mathrm{E}_{\mathrm{x}}{ }^{\mathrm{z}+1}$ is the $\mathrm{x}$ component of the electric field at the point $(\mathrm{x}, \mathrm{y}, \mathrm{z}+1)$. Similarly we can write the components of electric field at other points. $\mathrm{H}_{\mathrm{x}}, \mathrm{H}_{\mathrm{y}}, \mathrm{H}_{\mathrm{z}}$ are the $\mathrm{x}, \mathrm{y}$ and $\mathrm{z}$ components of the magnetic field at locations $(x, y, z) . H_{x}{ }^{x+1}$ is the $x$ component of the magnetic field at the location $(x+1, y, z)$. We can write the fields at other locations also. As an example, $E_{x}{ }^{x+1, y+1, z^{+1}}$ represents the $x$ component of electric field at the point $(x+1$, $y+1, z+1)$. In the FDTD software, we select a problem space, which is nothing but the space where the electromagnetic radiator (or scatterer) under test is studied. Space as well as the time is quantized. The whole space is thus divided into cubes of dimensions $\mathrm{x}=$ $\Delta x, y=\Delta y, z=\Delta z$, as shown in Fig. 3. The time is quantized as $\mathrm{t}=\mathrm{n} \Delta \mathrm{t}$ where $\mathrm{n}$ is the number of time steps. Usually the time step $\mathrm{n}$ is determined by an index (integer variable) variable $N$. For electric fields $n=N$, and for magnetic fields $n=N+1 / 2$ [20]. Hence usually the electric fields are calculated initially and used to calculate the magnetic fields in the next instant of time which are further used to calculate the electric fields in the next instant of time and the process goes on, till all the fields in the whole space are calculated. This is popularly known as leap frog fashion of calculating the fields.

\subsubsection{Equations formulation}

FDTD method utilizes a separate field formalism i.e.

$$
\begin{aligned}
& \mathrm{E}=\mathrm{E}^{\text {total }}=\mathrm{E}^{\text {total }}+\mathrm{E}^{\text {scattered }} \\
& \mathrm{H}=\mathrm{H}^{\text {total }}=\mathrm{H}^{\text {total }}+\mathrm{H}^{\text {scattered }}
\end{aligned}
$$

The idea behind using a separate field formalism is that, we can specify the incident field, so that the algorithm does the computations to find the scattered field. 
To see how the equations for the electric and magnetic fields are calculated and how the finite differencing is applied, let us consider formulation of a perfect conductor in FDTD method. The starting point for FDTD method is the curl equations. For clarity, let us write the Maxwell's equations once again

$$
\begin{aligned}
& \nabla x E=-\partial \mathrm{B} / \partial \mathrm{t} \\
& \nabla x H=-\partial \mathrm{D} / \partial \mathrm{t}+\mathrm{J}
\end{aligned}
$$

Utilizing the fact that $\mathrm{B}=\mu \mathrm{H}$ and $\mathrm{D}=\varepsilon \mathrm{E}, \quad$ (5.7) and (5.8) are re-written as

$$
\begin{gathered}
-\partial \mathrm{D} / \partial \mathrm{t}=-\frac{1}{\mu}(\nabla \times \mathrm{E})-\frac{\sigma *}{\mu} \mathrm{H} \\
\partial \mathrm{E} / \partial \mathrm{t}=-\frac{\sigma}{\varepsilon} \mathrm{E}+\frac{1}{\varepsilon}(\nabla \times \mathrm{H})
\end{gathered}
$$

Since we have considered the case of a perfect conductor, we have $\sigma^{*}=\sigma=0, \mu=\mu_{0}$ and $\varepsilon=\varepsilon_{0}$, Hence re-writing (5.9) and (5.10) we get,

$$
\begin{aligned}
& \frac{\partial E}{\partial t}=\frac{1}{\varepsilon o}(\nabla \times \mathrm{H}) \\
& \frac{\partial H}{\partial t}=-\frac{1}{\mu o}(\nabla \times \mathrm{E})
\end{aligned}
$$

By writing the above equations separately for $\mathrm{x}, \mathrm{y}$ and $\mathrm{z}$ components of the electric fields and magnetic field, we get

$$
\begin{aligned}
& \frac{\partial E_{x}}{\partial t}=\frac{1}{\varepsilon o}\left(\frac{\partial H_{z}}{\partial y}-\frac{\partial H_{y}}{\partial z}\right) \\
& \frac{\partial H_{x}}{\partial t}=-\frac{1}{\mu o}\left(\frac{\partial E_{y}}{\partial z}-\frac{\partial E_{z}}{\partial y}\right)
\end{aligned}
$$




$$
\begin{aligned}
& \frac{\partial E_{y}}{\partial t}=\frac{1}{\varepsilon o}\left(\frac{\partial H_{x}}{\partial z}-\frac{\partial H_{z}}{\partial x}\right) \\
& \frac{\partial H_{y}}{\partial t}=-\frac{1}{\mu o}\left(\frac{\partial E_{z}}{\partial x}-\frac{\partial E_{x}}{\partial z}\right) \\
& \frac{\partial E_{z}}{\partial t}=\frac{1}{\varepsilon o}\left(\frac{\partial H_{y}}{\partial x}-\frac{\partial H_{x}}{\partial y}\right) \\
& \frac{\partial H_{z}}{\partial t}=-\frac{1}{\mu o}\left(\frac{\partial E_{x}}{\partial y}-\frac{\partial E_{y}}{\partial x}\right)
\end{aligned}
$$

In case of a perfect conductor $E^{\text {scat }}=-E^{\text {inc }}$. Assuming that the problem space consists of only the conductor and free space, scattered fields can be calculated by specifying the incident fields. We apply the concept of differencing here. Finite difference replaces the derivatives with difference equations as shown below.

$$
\begin{aligned}
& \frac{\partial f}{\partial t} \equiv \lim _{\Delta t \rightarrow 0} \frac{f\left(x, t_{2}\right)-f\left(x, t_{1}\right)}{\Delta t} \approx \frac{f\left(x, t_{2}\right)-f\left(x, t_{1}\right)}{\Delta t} \\
& \frac{\partial f}{\partial x} \equiv \lim _{\Delta x \rightarrow 0} \frac{f\left(x_{2}, t\right)-f\left(x_{1}, t\right)}{\Delta x} \approx \frac{f\left(x_{2}, t\right)-f\left(x_{1}, t\right)}{\Delta x}
\end{aligned}
$$

Now (5.19) is applied to the right hand side and equation (5.20) is applied to the left hand side of the (5.13)-(5.18) respectively.

$$
\begin{aligned}
& \frac{E_{x}^{n}-E_{x}^{n-1}}{\Delta t}=\frac{1}{\varepsilon \circ}\left(\frac{\Delta H_{z}^{n-\frac{1}{2}}}{\Delta y}-\frac{\Delta H_{y}^{n-\frac{1}{2}}}{\Delta z}\right) \\
& \frac{E_{y}^{n}-E_{y}^{n-1}}{\Delta t}=\frac{1}{\varepsilon \circ}\left(\frac{\Delta H_{x}^{n-\frac{1}{2}}}{\Delta z}-\frac{\Delta H_{z}^{n-\frac{1}{2}}}{\Delta x}\right)
\end{aligned}
$$




$$
\begin{aligned}
& \frac{E_{z}^{n}-E_{z}^{n-1}}{\Delta t}=\frac{1}{\varepsilon \circ}\left(\frac{\Delta H_{y}^{n-\frac{1}{2}}}{\Delta x}-\frac{\Delta H_{x}^{n-\frac{1}{2}}}{\Delta y}\right) \\
& \frac{H_{x}^{n+\frac{1}{2}}-H_{x}^{n-\frac{1}{2}}}{\Delta t}=\frac{1}{\mu \circ}\left(\frac{\Delta E_{y}^{n}}{\Delta z}-\frac{\Delta E_{z}^{n}}{\Delta y}\right) \\
& \frac{H_{y}^{n+\frac{1}{2}}-H_{y}^{n-\frac{1}{2}}}{\Delta t}=\frac{1}{\mu \circ}\left(\frac{\Delta E_{z}^{n}}{\Delta x}-\frac{\Delta E_{x}^{n}}{\Delta z}\right) \\
& \frac{H_{z}^{n+\frac{1}{2}}-H_{z}^{n-\frac{1}{2}}}{\Delta t}=\frac{1}{\mu \circ}\left(\frac{\Delta E_{x}^{n}}{\Delta y}-\frac{\Delta E_{y}^{n}}{\Delta x}\right)
\end{aligned}
$$

Now, if we need the fields for the time instant $n+1$, we replace $n$ with $n+1$ in the $(5.21)-$ (5.26). Also terms like $\left(\frac{\Delta E_{z}^{n}}{\Delta x}\right)$ are further simplified as shown below.

$$
\begin{aligned}
& E_{x, i, j, k}^{n+1}=E_{x, i, j, k}^{n}+\frac{\Delta t}{\varepsilon \cdot \Delta y}\left(H_{z, i, j+1, k}^{n+\frac{1}{2}}-H_{z, i, j, k}^{n+\frac{1}{2}}\right)-\frac{\Delta t}{\varepsilon \cdot \Delta z}\left(H_{y, i, j, k+1}^{n+\frac{1}{2}}-H_{y, i, j, k}^{n+\frac{1}{2}}\right) \\
& E_{y, i, j, k}^{n+1}=E_{y, i, j, k}^{n}+\frac{\Delta t}{\varepsilon \cdot \Delta z}\left(H_{x, i, j, k+1}^{n+\frac{1}{2}}-H_{x, i, j, k}^{n+\frac{1}{2}}\right)-\frac{\Delta t}{\varepsilon \cdot \Delta x}\left(H_{z, i+1, j, k}^{n+\frac{1}{2}}-H_{z, i, j, k}^{n+\frac{1}{2}}\right) \\
& E_{z, i, j, k}^{n+1}=E_{z, i, j, k}^{n}+\frac{\Delta t}{\varepsilon \cdot \Delta x}\left(H_{y, i+1, j, k}^{n+\frac{1}{2}}-H_{y, i, j, k}^{n+\frac{1}{2}}\right)-\frac{\Delta t}{\varepsilon \cdot \Delta y}\left(H_{x, i, j+1, k}^{n+\frac{1}{2}}-H_{x, i, j, k}^{n+\frac{1}{2}}\right) \\
& H_{x, i, j, k}^{n+\frac{1}{2}}=H_{x, i, j, k}^{n-\frac{1}{2}}+\frac{\Delta t}{\mu \cdot \Delta z}\left(E_{y, i, j, k}^{n}-E_{y, i, j, k-1}^{n}\right)-\frac{\Delta t}{\mu \circ \cdot \Delta y}\left(E_{z, i, j, k}^{n}-E_{z, i, j-1, k}^{n}\right) \\
& H_{y, i, j, k}^{n+\frac{1}{2}}=H_{y, i, j, k}^{n-\frac{1}{2}}+\frac{\Delta t}{\mu \cdot \Delta x}\left(E_{z, i, j, k}^{n}-E_{z, i-1, j, k}^{n}\right)-\frac{\Delta t}{\mu \circ . \Delta z}\left(E_{x, i, j, k}^{n}-E_{x, i, j, k-1}^{n}\right)
\end{aligned}
$$




$$
H_{z, i, j, k}^{n+\frac{1}{2}}=H_{z, i, j, k}^{n-\frac{1}{2}}+\frac{\Delta t}{\mu \cdot \Delta y}\left(E_{y, i, j, k}^{n}-E_{y, j, j-1, k}^{n}\right)-\frac{\Delta t}{\mu \circ . \Delta x}\left(E_{z, i, j, k}^{n}-E_{z, i-1, j, k}^{n}\right)
$$

From the above equations [20], [21] one can easily observe how the electric field at the nth instant is used to calculate the magnetic field at $(n+1 / 2)$ instant of time and this in term is used to calculate the electric field at the $(n+1)$ instant. This is how the software calculates the corresponding fields. However, inorder to ensure that the results we get after simulation are reasonably accurate, some considerations like the cell size, time stepsize, incident field specification, etc. need to be made. For example, if we consider the time step, it is calculated using the Courant condition.

$$
v \Delta t \leq \frac{1}{\sqrt{\frac{1}{(\Delta x)^{2}}+\frac{1}{(\Delta y)^{2}}+\frac{1}{(\Delta z)^{2}}}}
$$

$\mathrm{v}$ is the velocity of the electromagnetic wave in he medium

$\Delta \mathrm{t}$ is the time step

$\Delta \mathrm{x}, \Delta \mathrm{y}, \Delta \mathrm{z}$ are the dimensions of the cell.

Also the cell size must be less than one tenth of the wavelength of the electromagnetic wave (in that medium). This implies ten cells per wavelength. Some applications demand even smaller cell size. But in most cases $\lambda / 10$ makes a good choice to get reasonably accurate results [20], [21].

\subsubsection{Merits and demerits}

FDTD method has become one of the most widely used techniques. As it is a time domain method, it can encompass a wide frequency range with single run. It is easy to 
understand and implement in software [2]. Merits of FDTD method can be summarized as follows

- We can model any arbitrarily shaped three dimensional geometry ( aircrafts, human bodies, satellites etc.).

- Performance can be obtained for a broad range of frequencies with single simulation run (as it is a time domain method).

- Objects with almost any value of conductivity can be specified and modeled.

- Both electric and magnetic materials can be modeled.

- FDTD method has very good absorbing boundary conditions ( $\mathrm{ABCs}$ ) like the Liao, PML etc;

- FDTD method provides a display as to how the actual radiation looks at various time instants. This allows the user to understand about the current densities at various locations.

- Another important advantage is that FDTD method provides the sub grid feature, which can be used in the region of the structure where greater accuracy is desired.

- FDTD method also allows us to mention the type of feed (stimulus). It has many types of stimuli (Gaussian, modulated Gaussian etc.) to select from.

- Further, FDTD method offers some basic geometries like the sphere, the cylinder, wire etc.

Demerits of FDTD method can be summarized as

- FDTD method requires huge amount of memory. Also the processor must be fast enough, for the calculations to run faster. 
- The selection of cell size must be made as small as possible. Though it increases accuracy, it requires a lot of time for the to run. Hence tradeoff between cell size and accuracy must be made.

- If the point where the field has to be calculated is a bit far more problem space might have to be selected. XFDTD software can calculate far field values but it requires post processing which takes a lot of time.

\subsubsection{System requirements for XFDTD software}

XFDTD from Remcom is the software used in this thesis work. The minimum system requirements for XFDTD software are [2]

- 32 MB RAM, 128 MB

- $1024 \times 768$ resolution

- 256 color display

- 10 MB Hard Drive space for executable files

- $300 \mathrm{MB}$ Hard Drive for full installation of all example files

\subsubsection{Reason for choosing FDTD method}

The most important reason for selecting FDTD method is that it can analyze the structure for a wide range of frequencies in one simulation run, where as this is not possible with MoM (method of moments). 


\section{CHAPTER 6}

\section{METHODOLOGY}

\subsection{Description}

The work done in this thesis can be divided into three parts. The first part being the study of diamond dipole, the second being the analysis of modified diamond dipole and the third part comprising of the study of slot loaded modified diamond dipole. The antennas are analyzed using FDTD method. Initially a diamond dipole as in [2] is analyzed (for completeness). Next, modified diamond dipole with various radius of curvatures of the base (of triangular arms) region and with different flare angles are constructed and analyzed. Next, a comparison of diamond dipole and modified diamond dipole is made. Lastly, modified diamond dipole is loaded with slots and the behavior of the antenna is studied for various slot dimensions. Also the slot loaded modified diamond dipole is compared with modified diamond dipole analyzed in part II.

Finally two new antenna designs (1) modified diamond dipole (2) slot loaded modified diamond dipole are proposed.

\subsection{Part I Diamond dipole}

Diamond dipole antenna for ultra-wideband communication consists of two triangular arms. The diamond dipole antenna analyzed here is same as in [2], except for a few changes in the dimensions of the dielectric layer. A dielectric substrate having the dimensions $79 \times 54 \times 1.5 \mathrm{~mm}^{3}$ and a relative permittivity of 4.7 is constructed and the antenna is centered on the substrate. The antenna with dimensions is shown in Fig. 6. To analyze the antenna using XFDTD software, we need to select a problem space large enough to fit the antenna and allow some boundary (Liao boundary) around the antenna. 


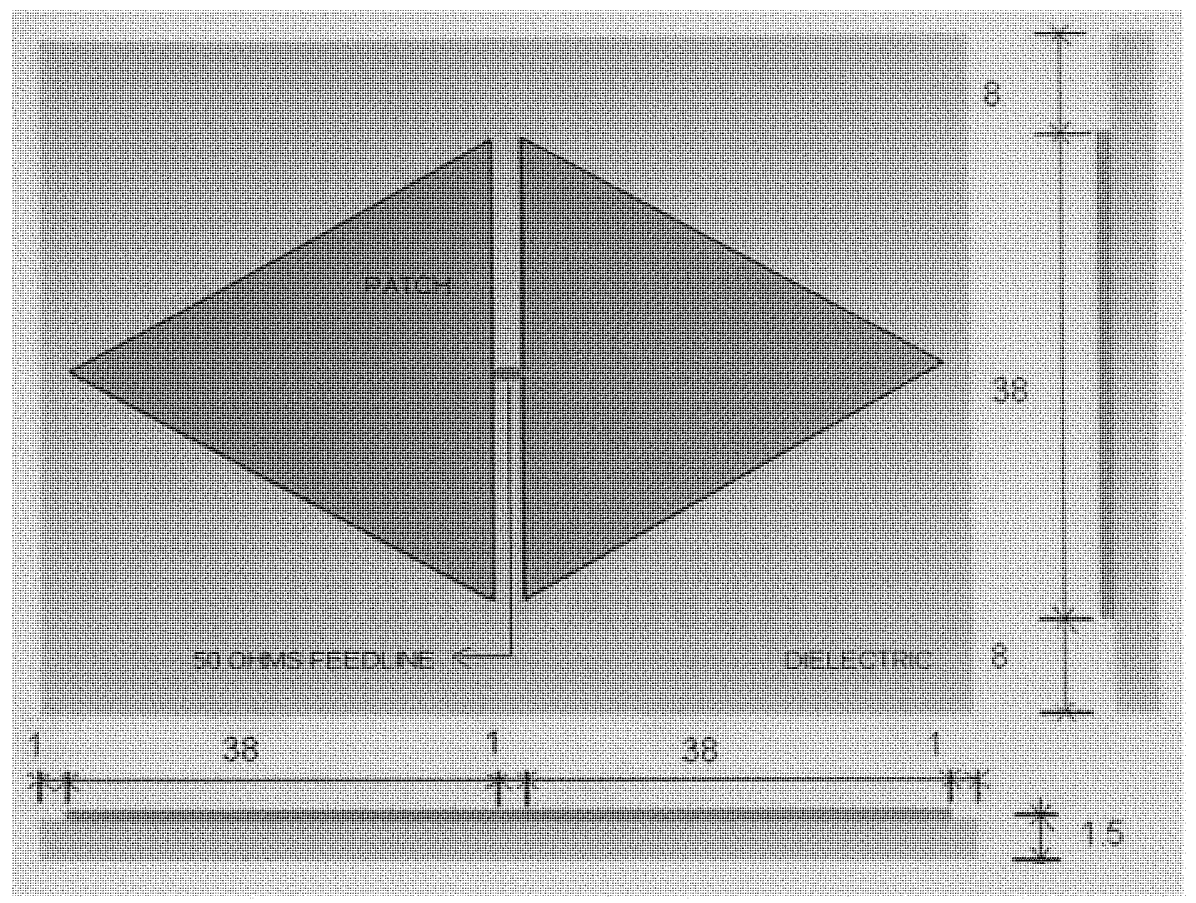

Fig. 6. Diamond dipole antenna with dimensions.

From the study done in chapter IV, the antenna geometry is to be constructed using Yee cells. Hence, we need to select dimensions of the cell. Ten cells per wavelength is a good choice $(\lambda / 10)$ to start with [22], [23]. The cell dimensions are selected as $\Delta x=\Delta y=1.0$ $\mathrm{mm}, \Delta \mathrm{z}=0.5 \mathrm{~mm}$. The cell dimensions are specified as shown in a window shown in Fig.

7.

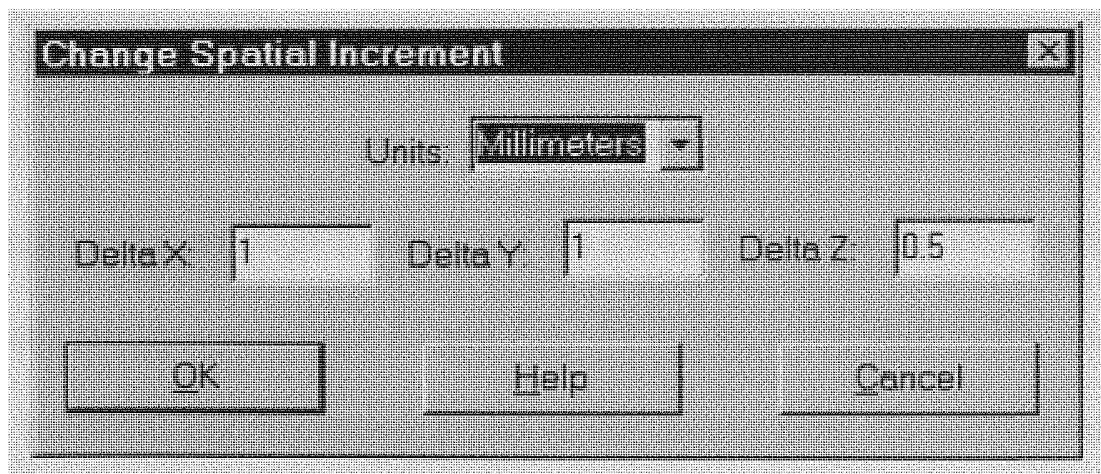

Fig. 7. Cellular dimensions in XFDTD software. 


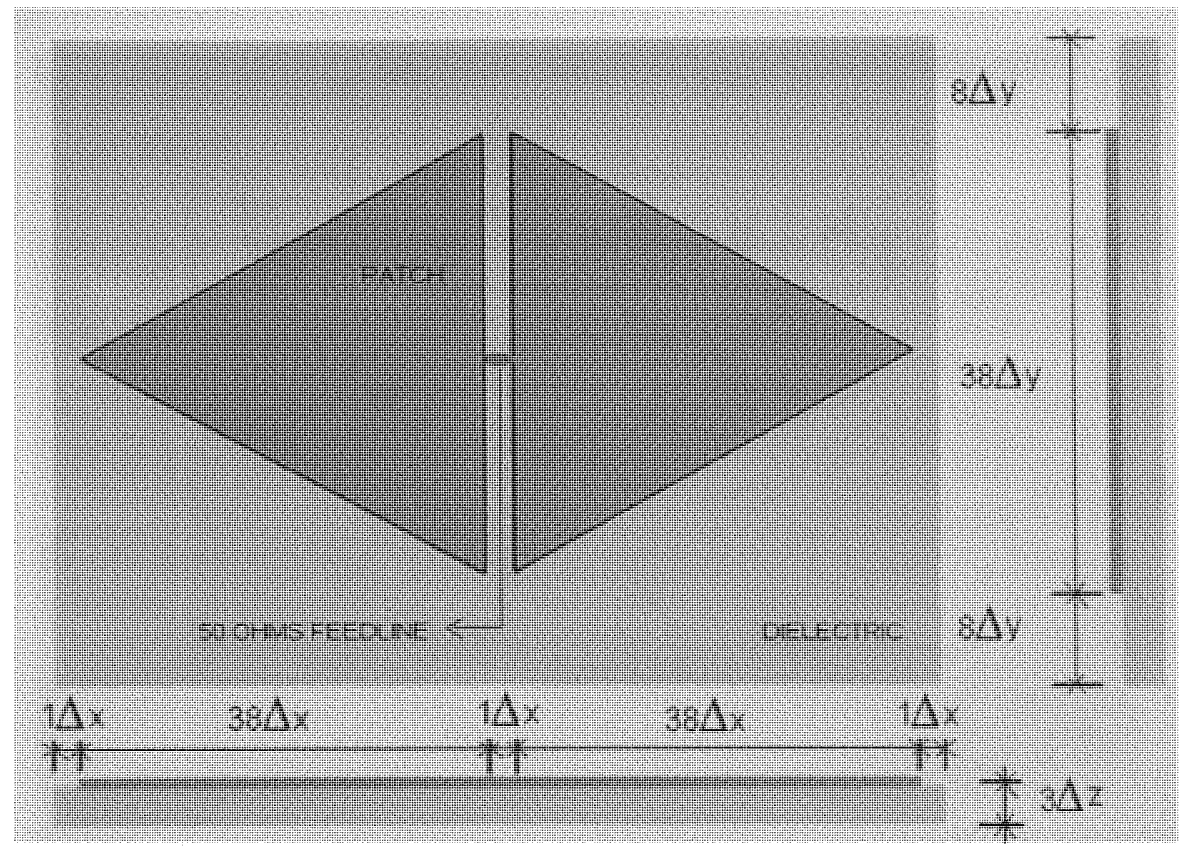

Fig. 8. Cellular dimensions of diamond dipole antenna.

Fig. 8 shows diamond dipole in terms of cellular dimensions. According to the cellular dimensions mentioned in Fig. 8, the antenna is constructed in XFDTD software. The geometry of the antenna in XFDTD software looks as in Fig. 9 and Fig. 10. The three dimensional view of the antenna is shown in Fig. 11. Liao's absorbing boundary condition, shown in Fig. 12 is selected for this purpose. The boundary extends to 15 cells on all the sides of the antenna geometry. Modulated Gaussian pulse has been selected for exciting the antenna. The time step as calculated by XFDTD is 0.001362 nano seconds. A pulse width of 512 time steps is taken and the simulation is run for 10,000 time steps. The source details are shown in Fig. 13 and Fig. 14 shows a snapshot of the window to enter the source location. Fig. 15 shows a plot of the source used for excitation. Fig. 16 and Fig. 17 show plots of return loss and input impedance calculated by XFDTD software. 


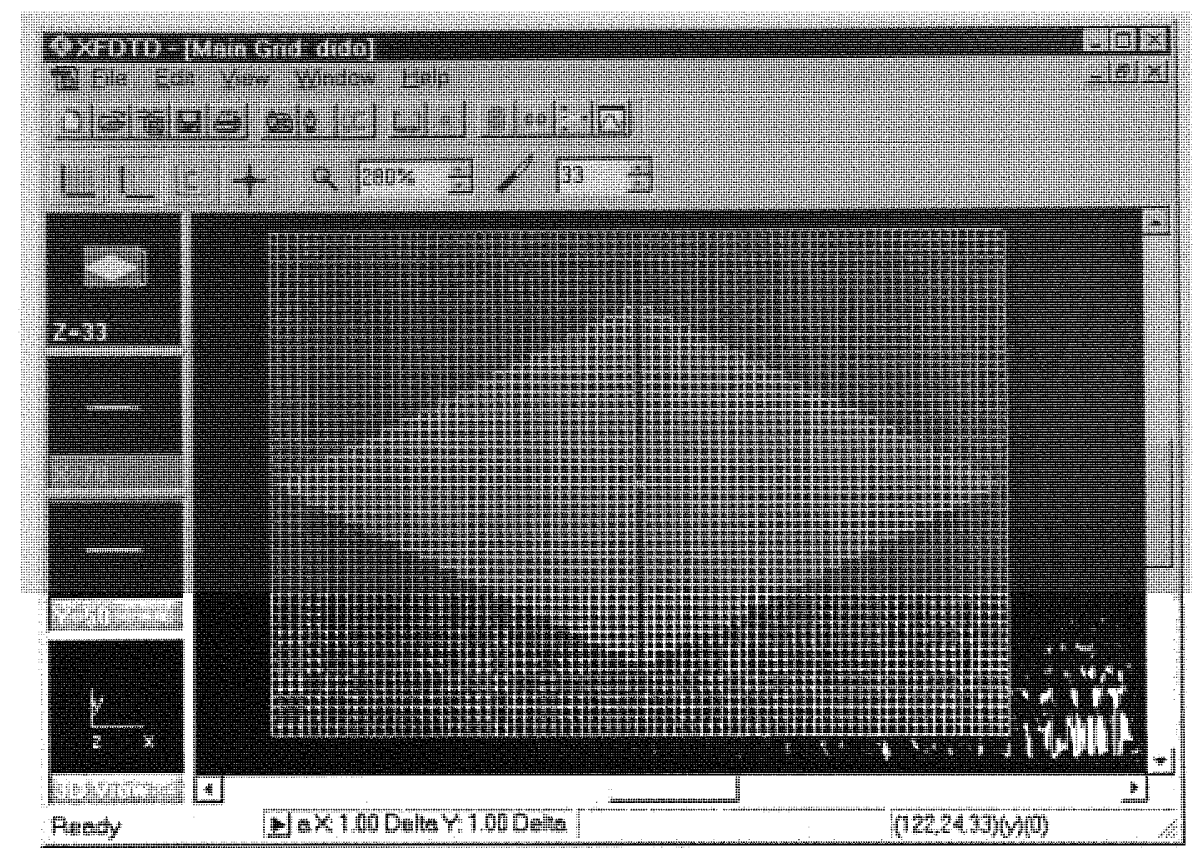

Fig. 9. Layer $z=33$ (xy plane) of diamond dipole in XFDTD software.

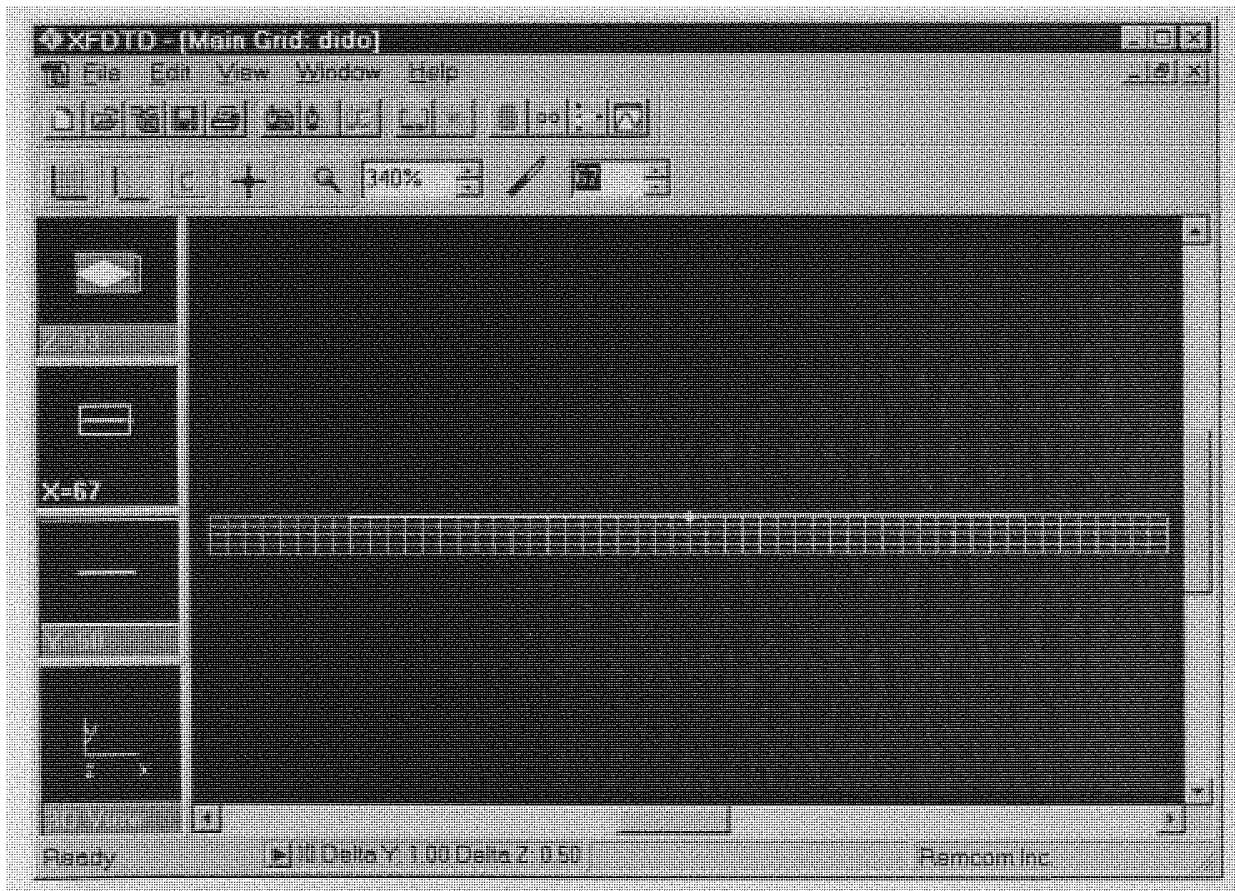

Fig. 10. Layer $\mathrm{x}=67$ ( $\mathrm{yz}$ plane) of diamond dipole in XFDTD software. 


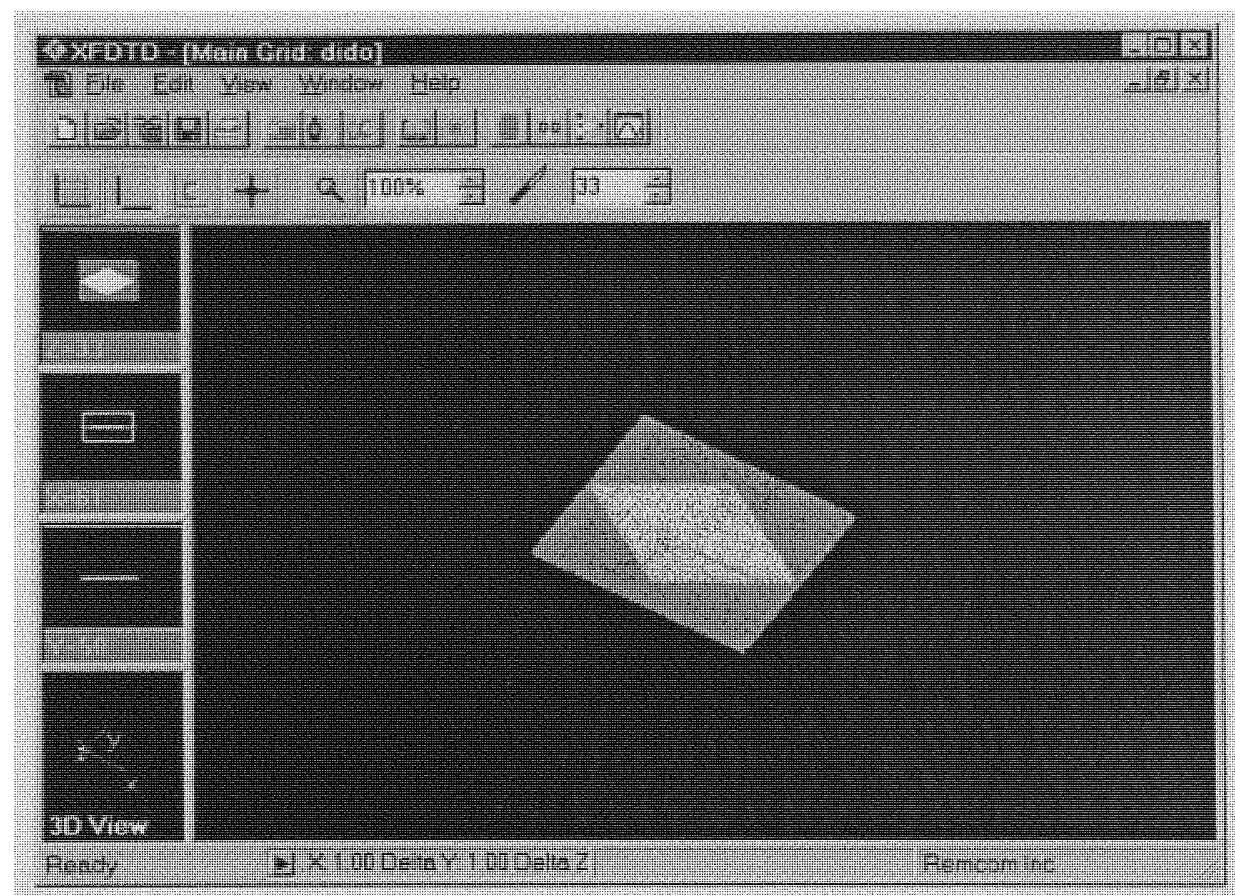

Fig. 11. 3-Dimensional view of diamond dipole in XFDTD software.



Fig. 12. Boundary condition selection in XFDTD software. 


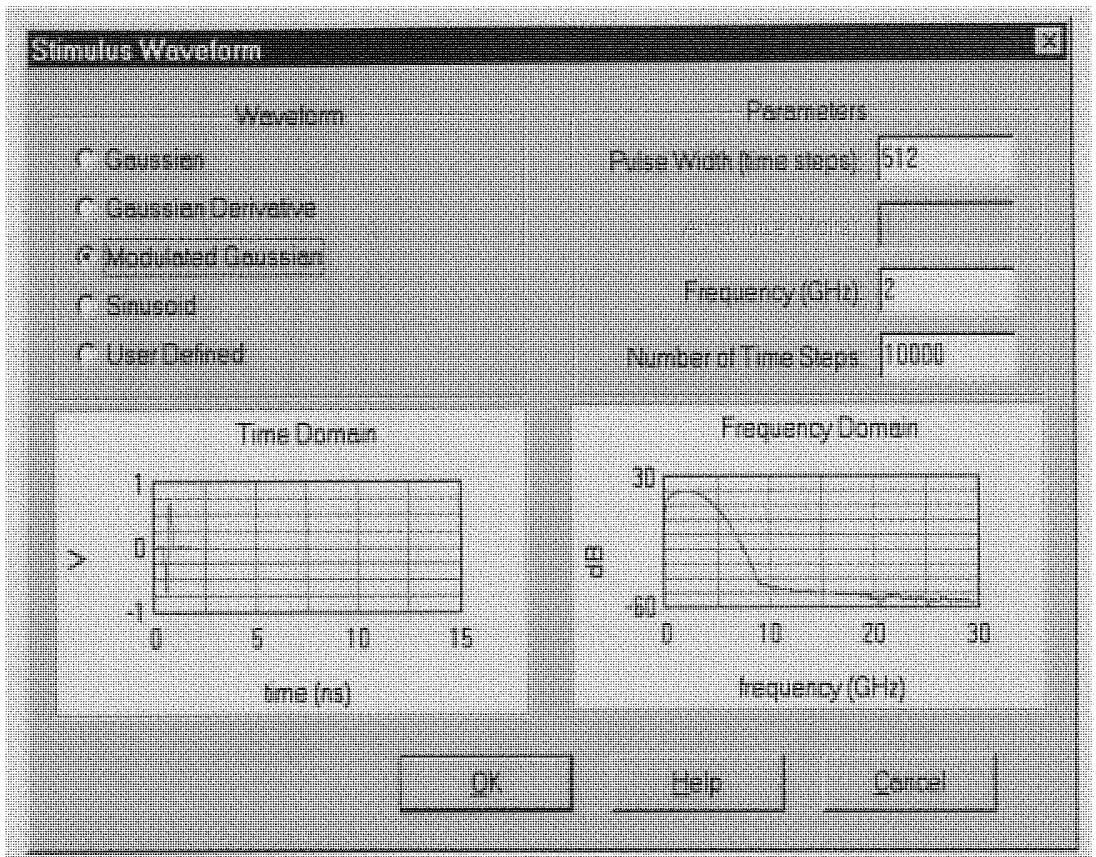

Fig. 13. Details of the modulated Guassian pulse in XFDTD software.

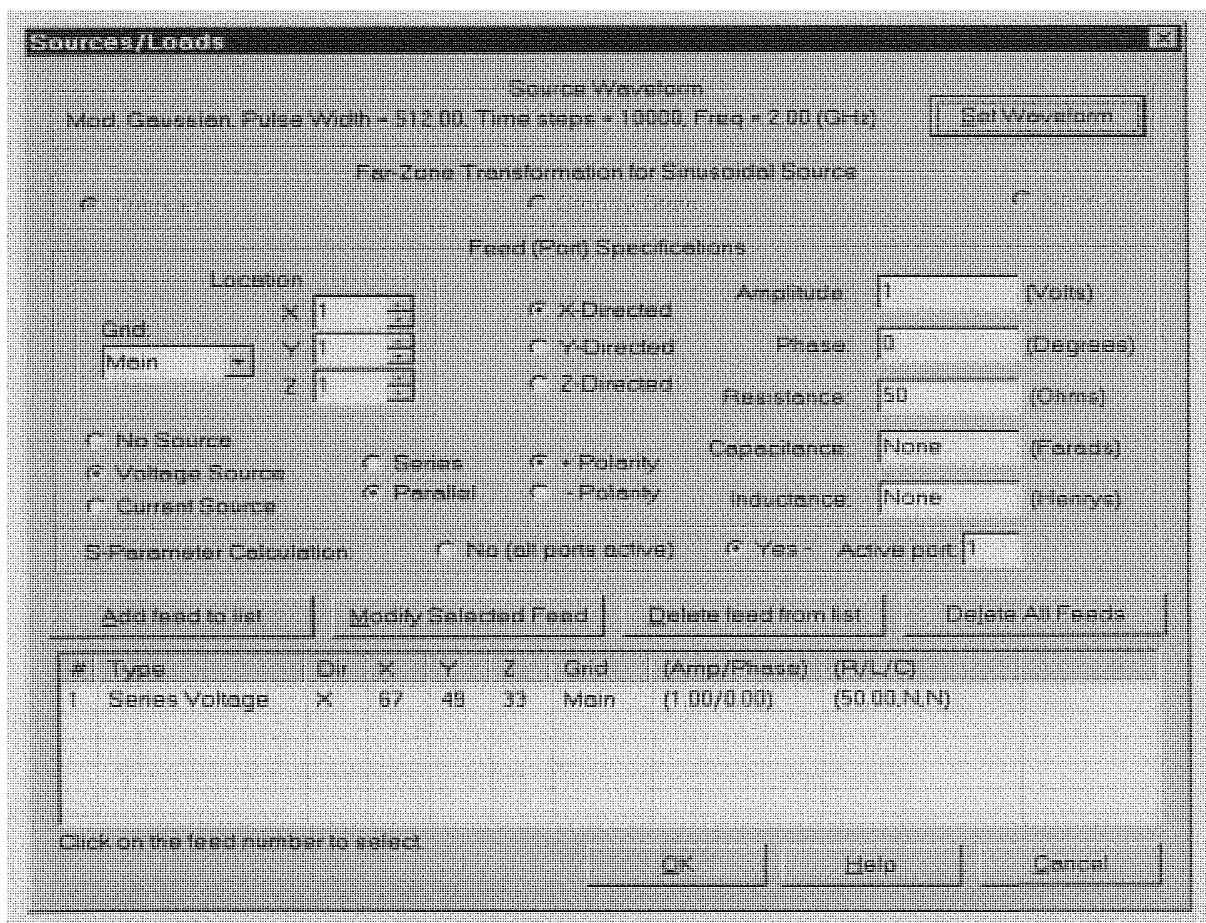

Fig. 14. Details of source in XFDTD software. 


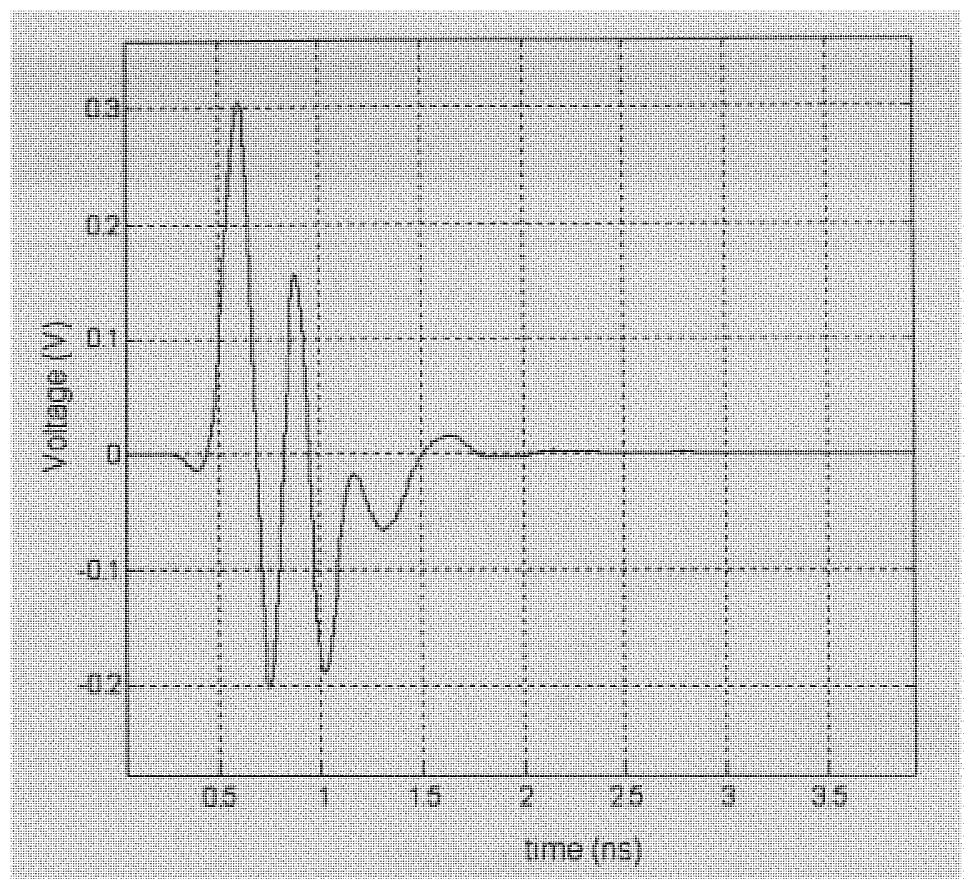

Fig. 15. Time domain representation of modulated Gaussian pulse used as a source.

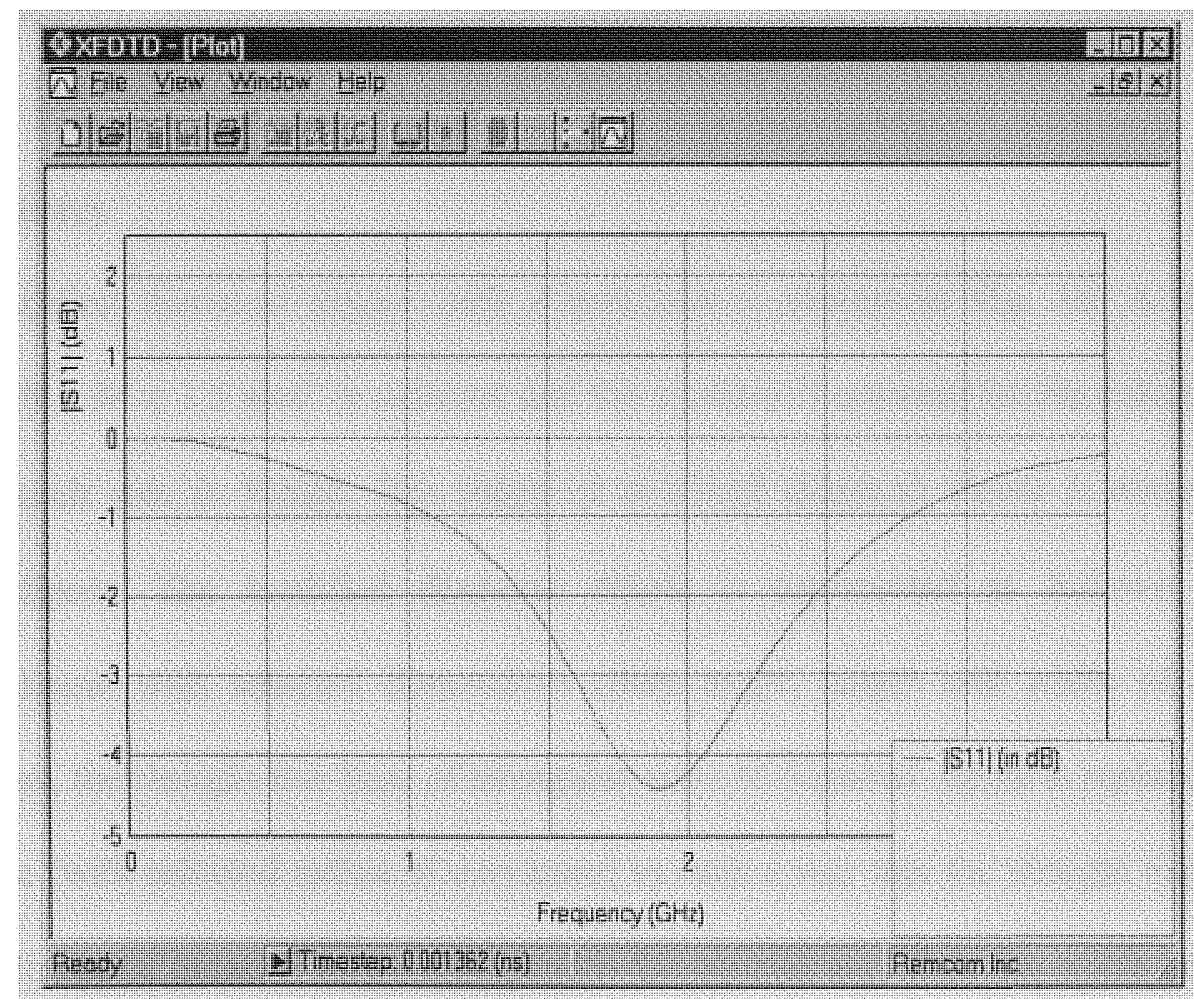

Fig. 16. Frequency Vs. return loss for diamond dipole antenna. 


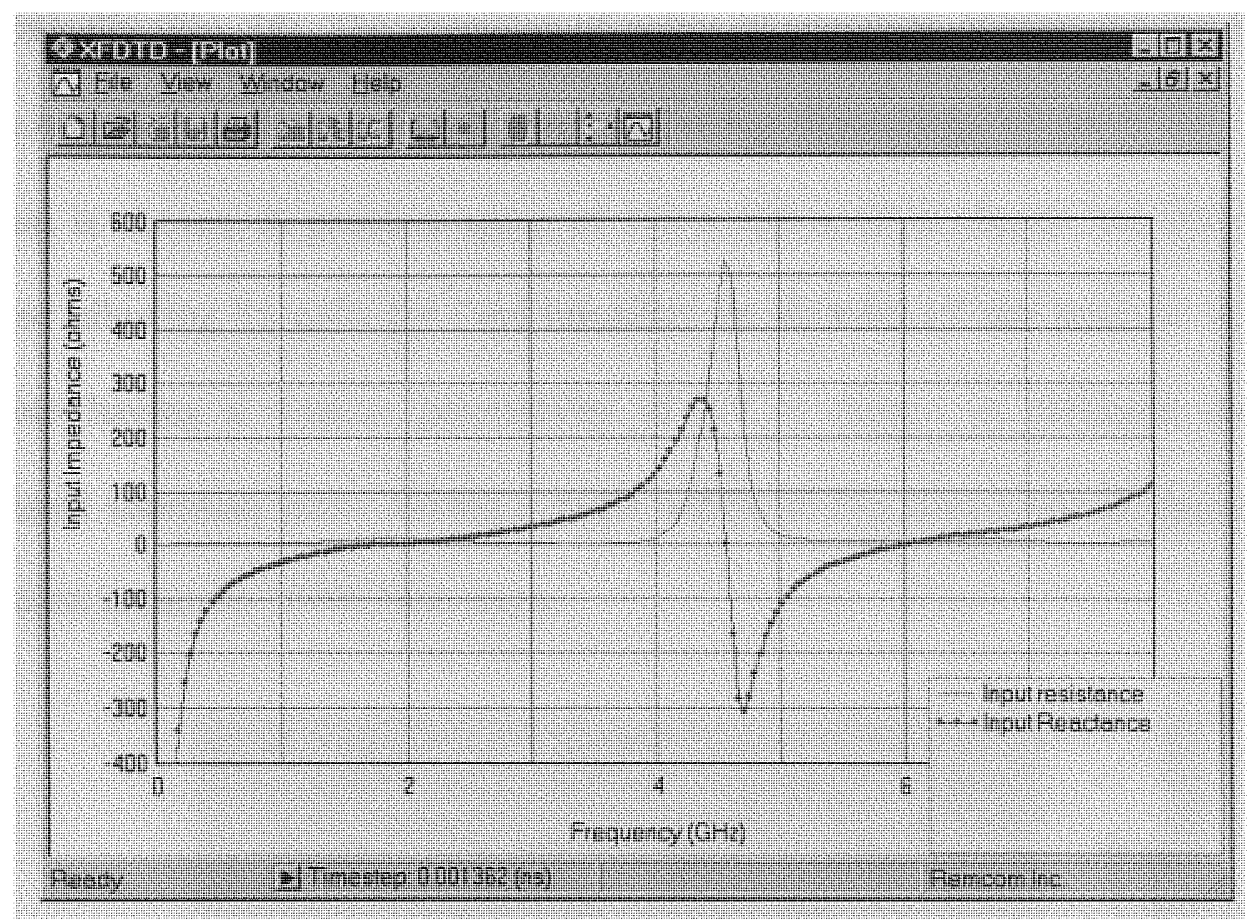

Fig. 17. Frequency Vs. input impedance for diamond dipole antenna.

\subsection{Part II Modified diamond dipole}

\subsubsection{Geometry}

Modified diamond dipole with dimensions is shown in Fig. 18. A dielectric substrate $79 \times 54 \times 1.5 \mathrm{~mm}^{3}$ is selected and the antenna is centered on the substrate. The length of each arm is $38 \mathrm{~mm}$. The shape of modified diamond dipole is derived from diamond dipole. The modified diamond dipole is constructed as follows: Initially diamond dipole with selected dimensions (flare angle, length and width of arms) is drawn. Next, the straight line base of triangular arms of diamond dipole is replaced with a circular arc (with selected radius of curvature) in such a way that the straight line base of diamond dipole (if present) forms a tangent to the circular arc. Thus the width becomes a function of flare angle and radius of curvature of the base arc and is generated 
automatically (Fig. 18). The alteration of radius of curvature of base and the flare angle are shown in Fig. 20 and Fig. 21. A cell having dimensions of $\Delta x=\Delta y=1 \mathrm{~mm}, \Delta z$ $=0.5 \mathrm{~mm}$ is chosen. A problem space of $140 \times 100 \times 65$ is chosen and the antenna is centered allowing 15 cells for Liao boundary on all sides of the antenna. A dielectric substrate having a relative permittivity of 4.7 is chosen as dielectric material. A modulated Gaussian pulse having a pulse width of 512 time steps is chosen and the simulation is run for 10000 time steps.

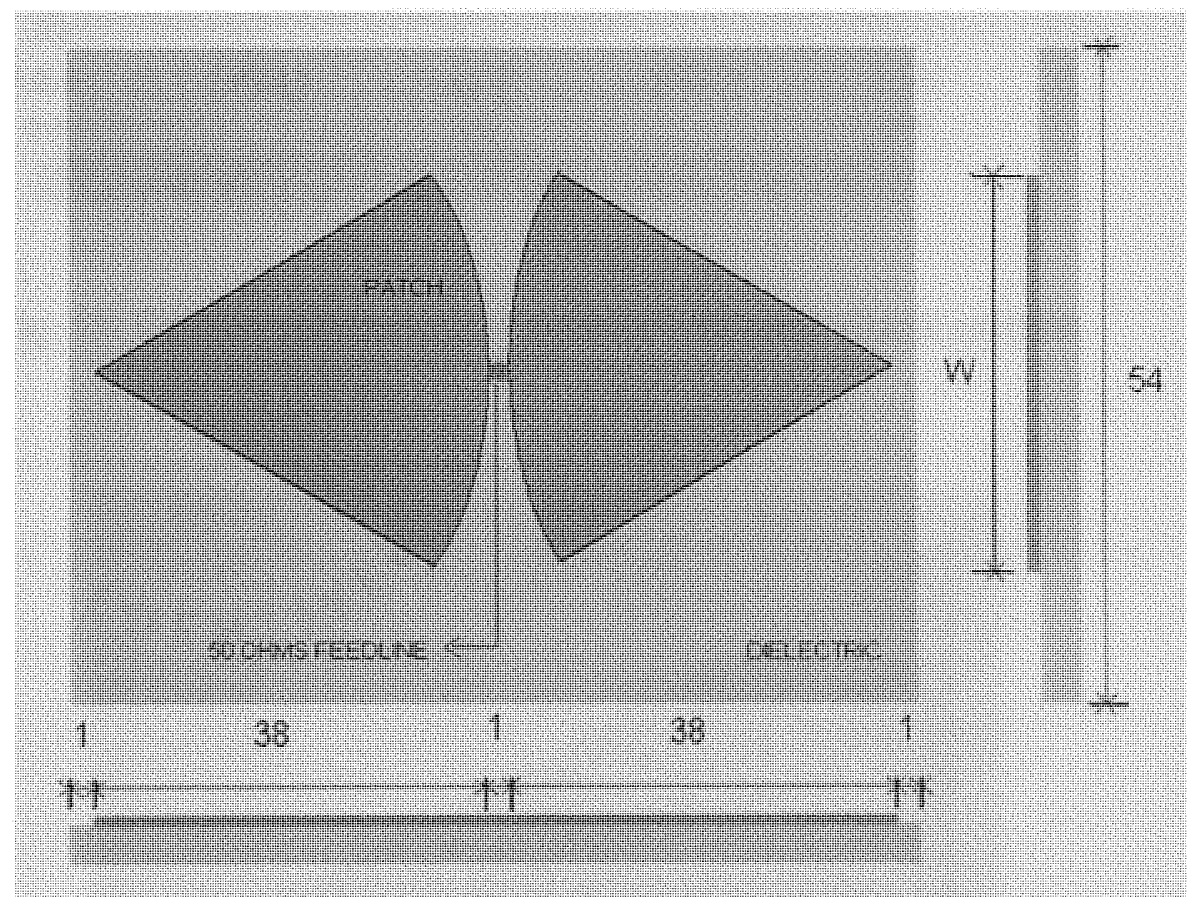

Fig. 18. Modified diamond dipole.

(all dimensions are in $\mathrm{mm}$ )

To study the modified diamond dipole, flare angle and radius of curvature of the bas of triangular arms are selected as parameters and 9 antenna designs are selected which are tabulated in Table 1. 


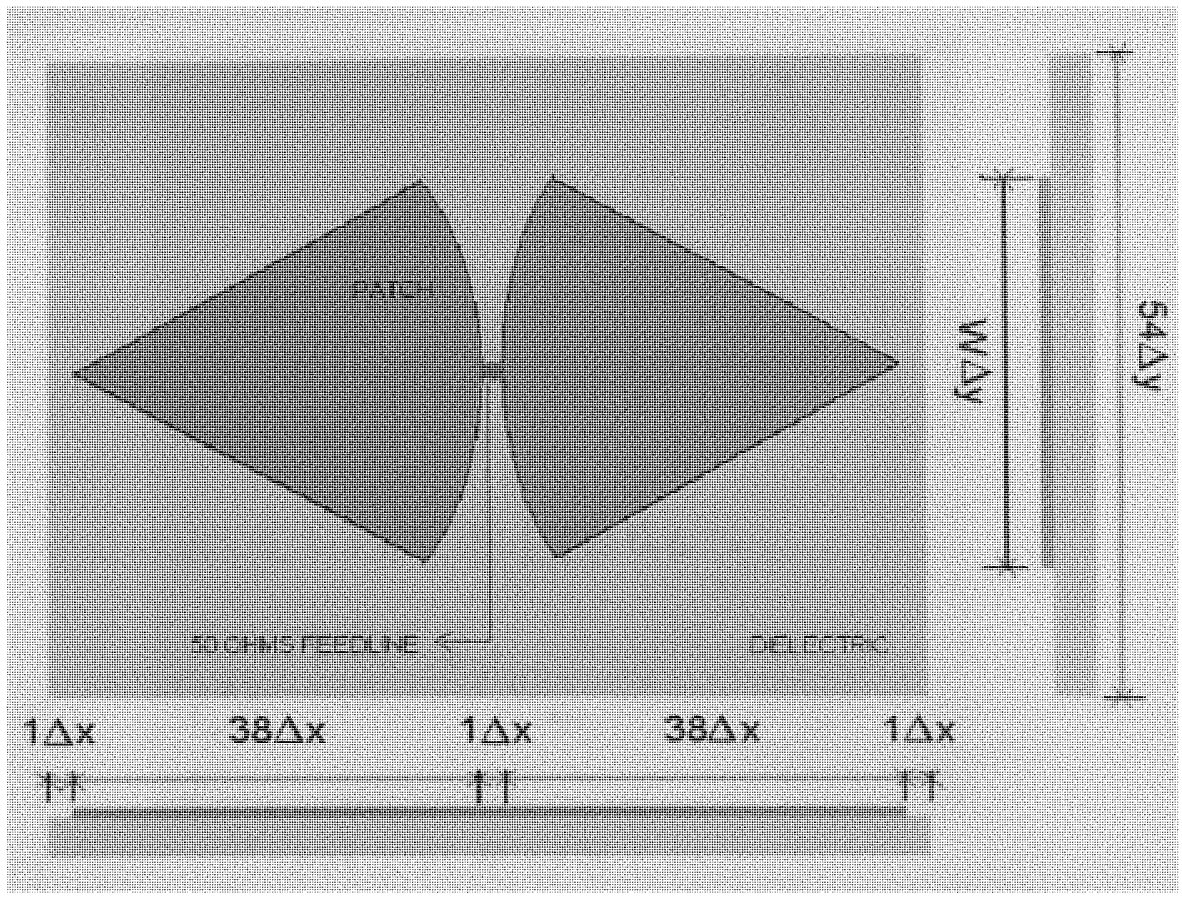

Fig. 19. Cellular dimensions of modified diamond dipole antenna.

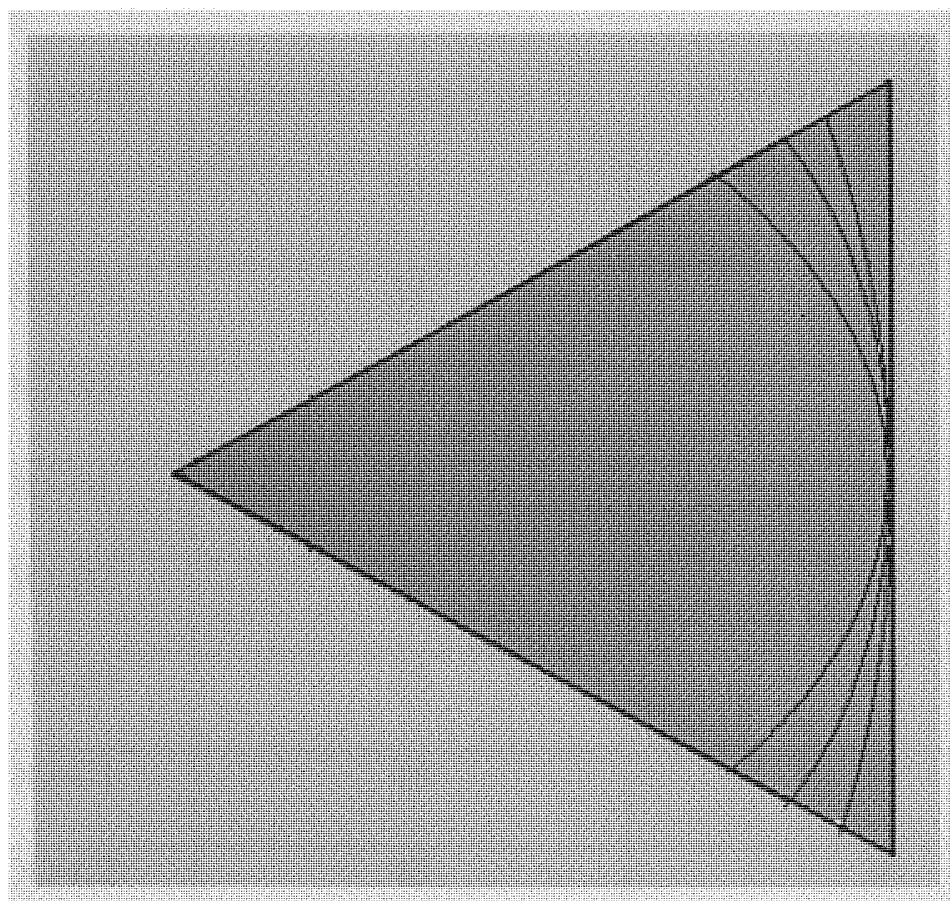

Fig. 20. Radius of curvature alteration of modified diamond dipole. 


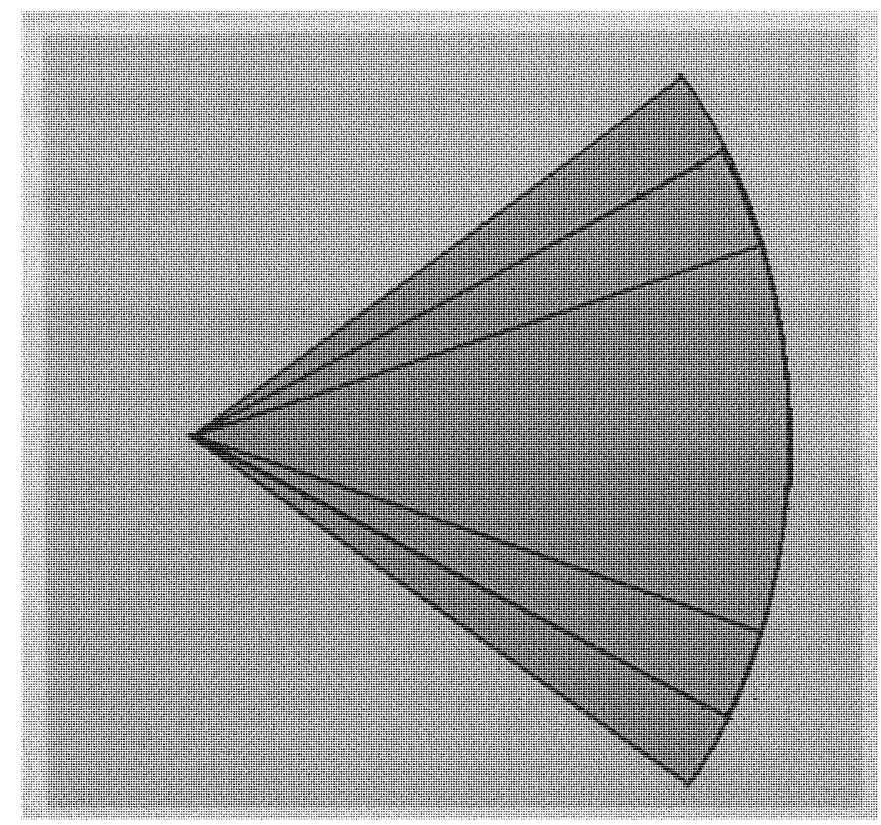

Fig. 21. Flare angle alteration of modified diamond dipole.

Table 1

Information about the modified diamond dipole antenna models

\begin{tabular}{|c|c|c|}
\hline Antenna model & Semi-flare angle $(\propto / 2)^{0}$ & Radius of curvature $(\mathrm{R} \mathrm{mm})$ \\
\hline MDIDO-A1 & 27 & 20 \\
\hline MDIDO-A2 & 27 & 30 \\
\hline MDIDO-A3 & 27 & 40 \\
\hline MDIDO-B1 & 35 & 20 \\
\hline MDIDO-B2 & 35 & 30 \\
\hline MDIDO-B3 & 35 & 40 \\
\hline MDIDO-C1 & 45 & 20 \\
\hline MDIDO-C2 & 45 & 30 \\
\hline MDIDO-C3 & 45 & 40 \\
\hline
\end{tabular}




\subsubsection{Modified diamond dipole, model MDIDO-A1}

Initially MDIDO-A1 antenna, is constructed in XFDTD software as shown in Fig. 22. A

3-D view is shown in Fig. 23.

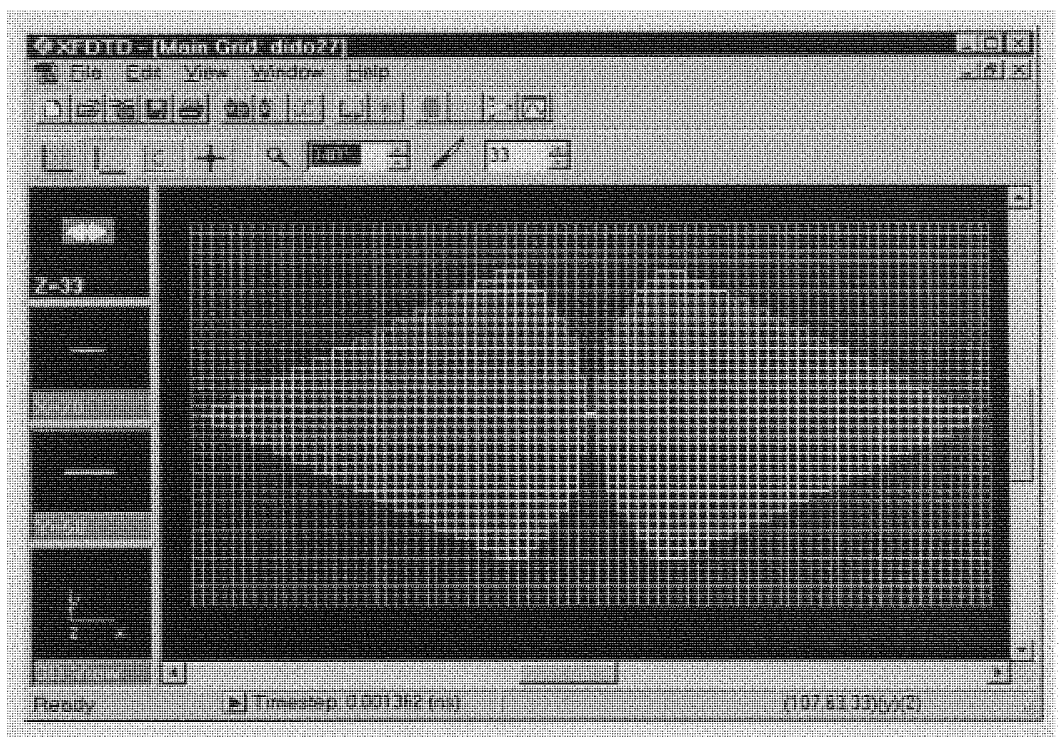

Fig. 22. Layer $\mathrm{z}=33$ (xy plane) of MDIDO-A1 in XFDTD software.

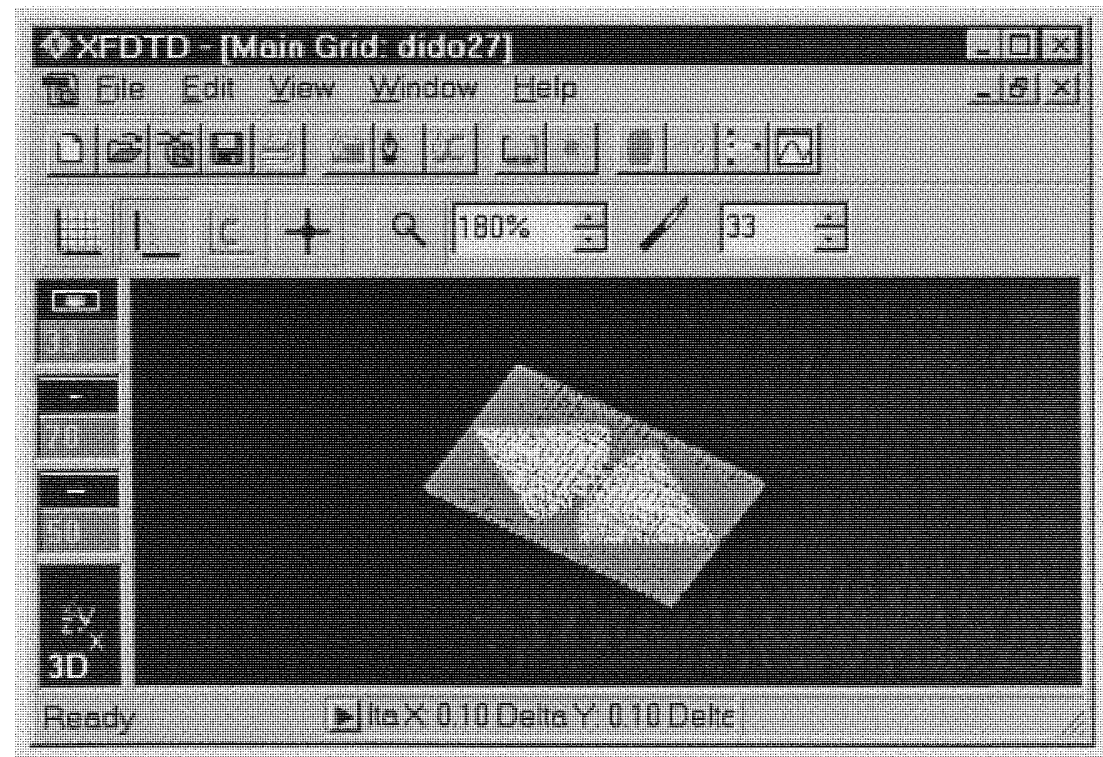

Fig. 23. 3-Dimensional view of MDIDO-A1 in XFDTD software. 
Fig. 24 and Fig. 25 show variation of return loss and input impedance with frequency respectively.

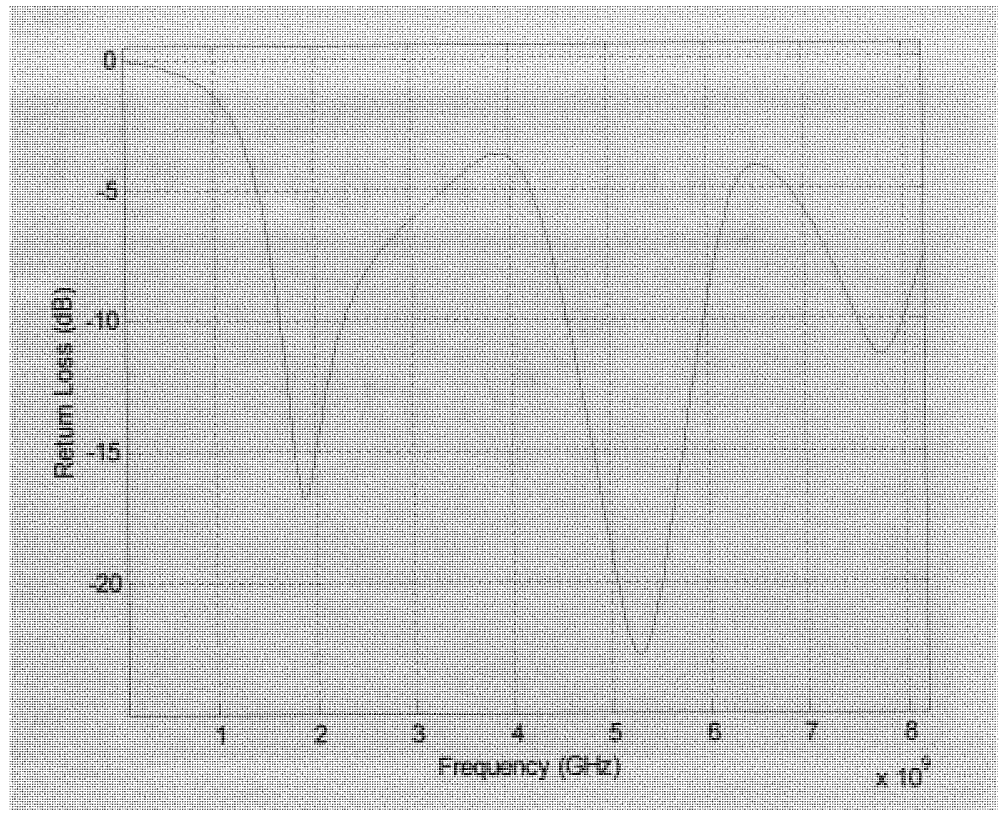

Fig. 24. Frequency Vs. return loss for MDIDO-A1.

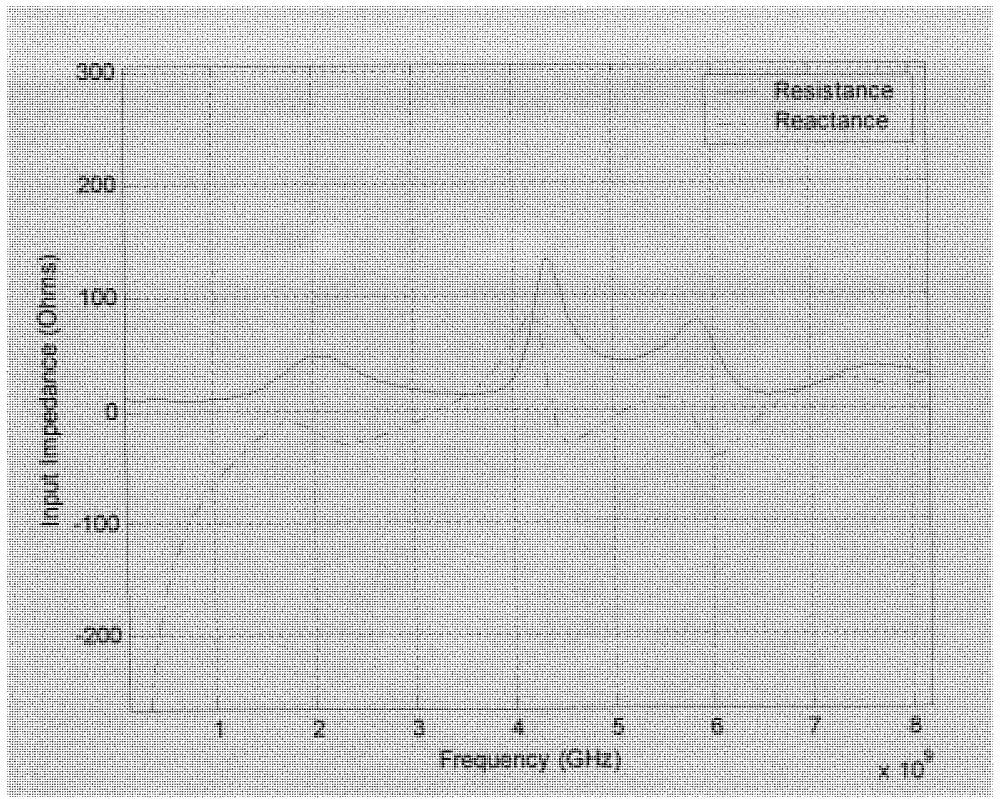

Fig. 25. Frequency Vs. input impedance for MDIDO-A1. 


\subsubsection{Modified diamond dipole, model MDIDO-B1}

MDIDO-B1 antenna is constructed in XFDTD software as shown in Fig. 26. A 3-D view is shown in Fig. 27.

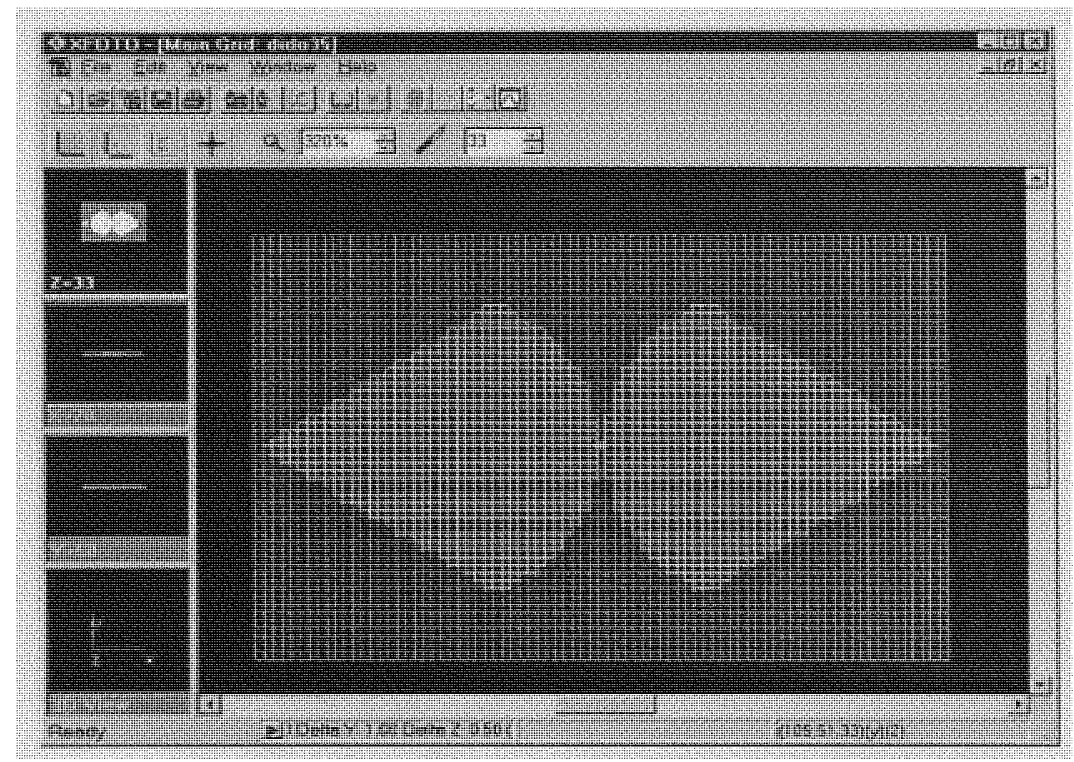

Fig. 26. Layer $\mathrm{z}=33$ (xy plane) of MDIDO-B1 in XFDTD software.

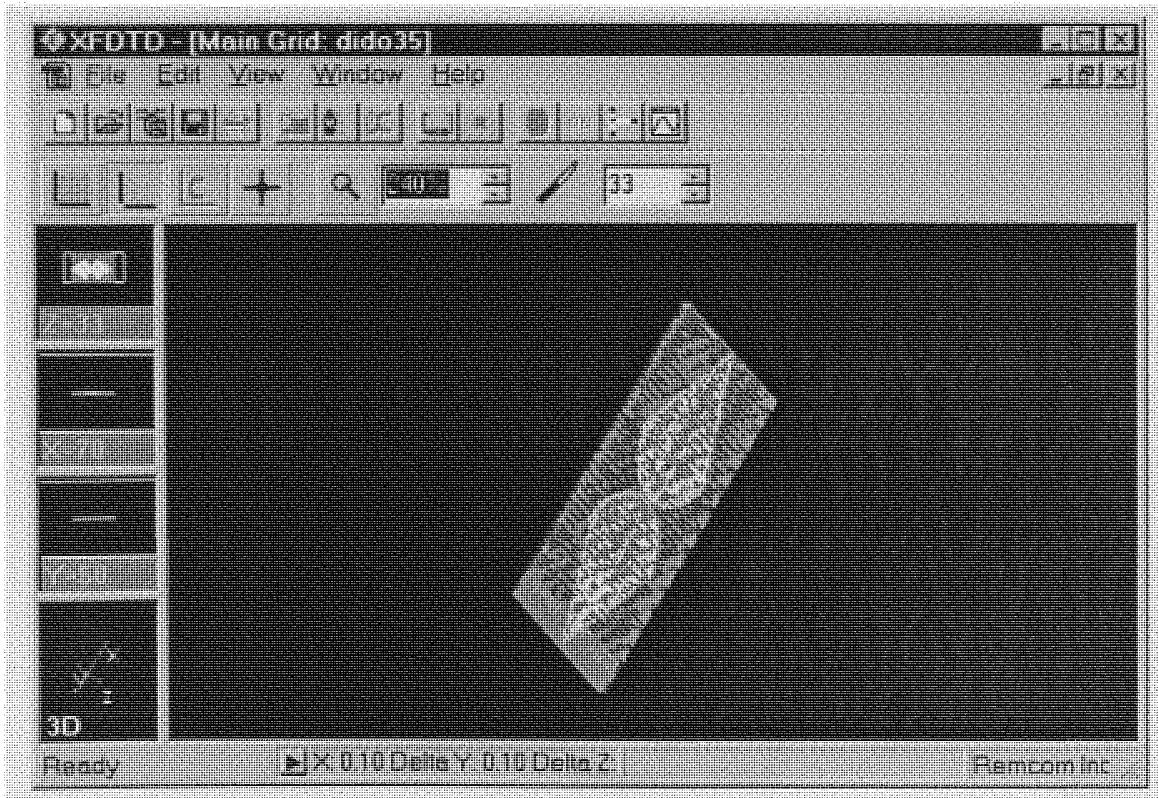

Fig. 27. 3-Dimensional view of MDIDO-B1 in XFDTD software. 
Fig. 28 and Fig. 29 show variation of return loss and input impedance with frequency respectively.

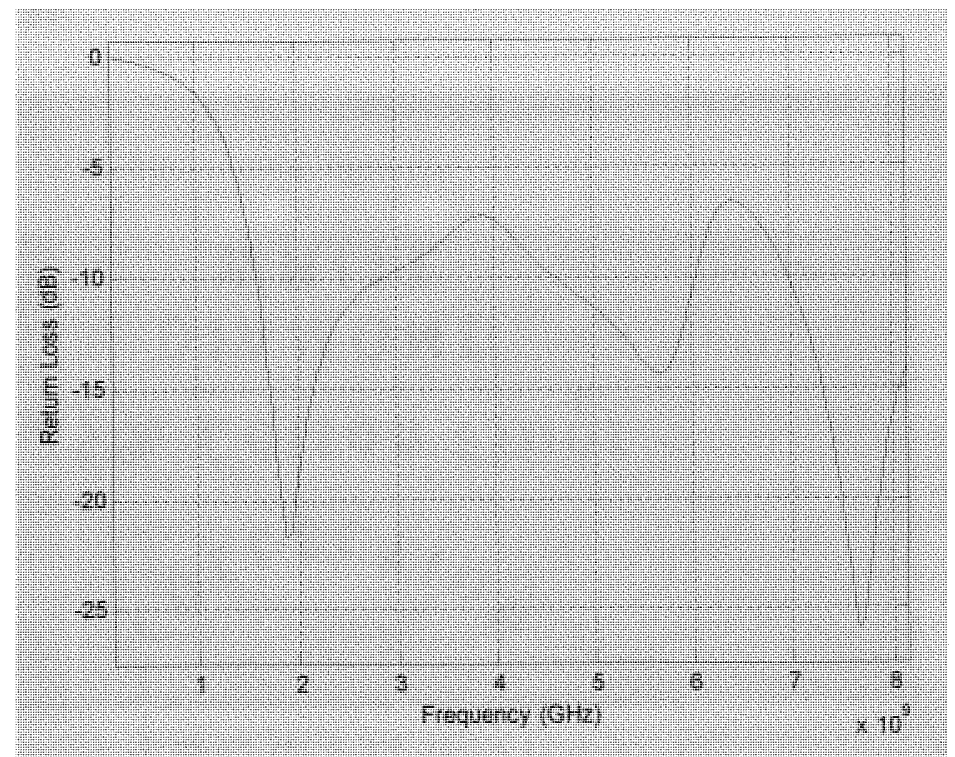

Fig. 28. Frequency Vs. return loss for MDIDO-B1.

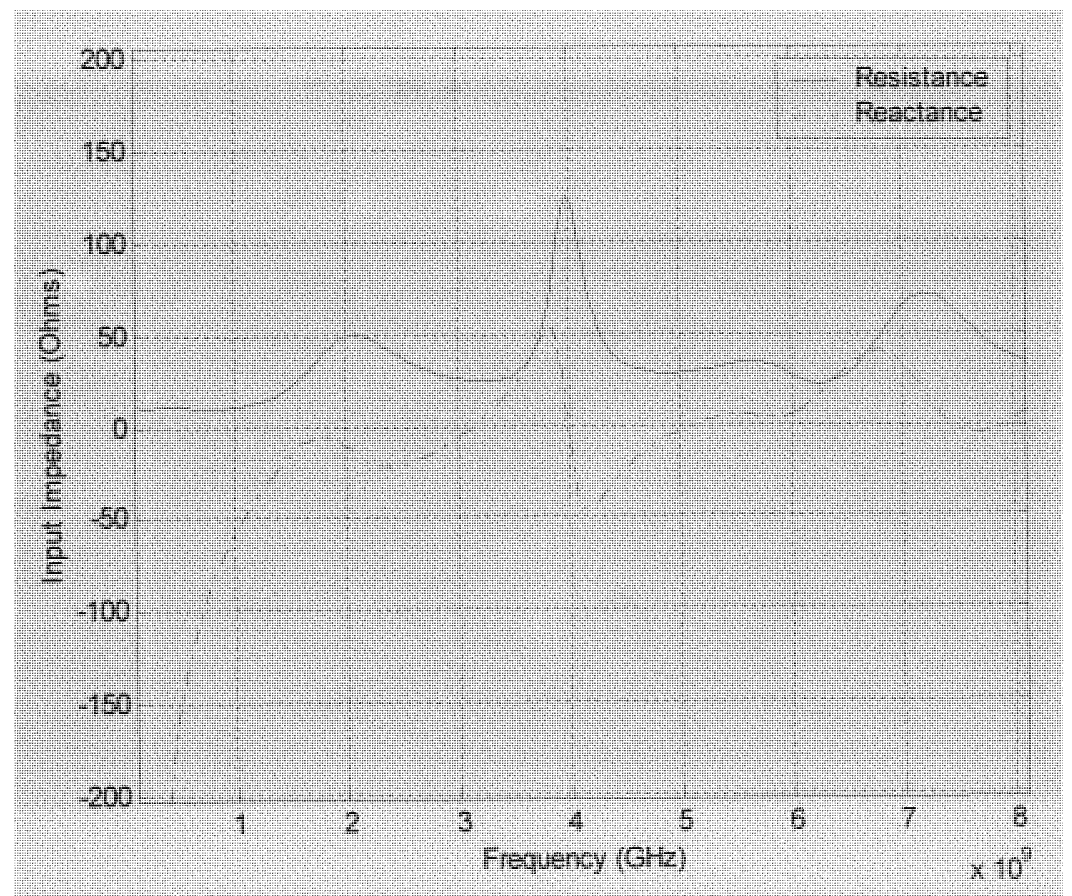

Fig. 29. Frequency Vs. input impedance for MDIDO-B1. 


\subsubsection{Modified diamond dipole, model MDIDO-C1}

MDIDO-C1 antenna is constructed in XFDTD software as shown in Fig. 30. A 3-D view is shown in Fig. 31.

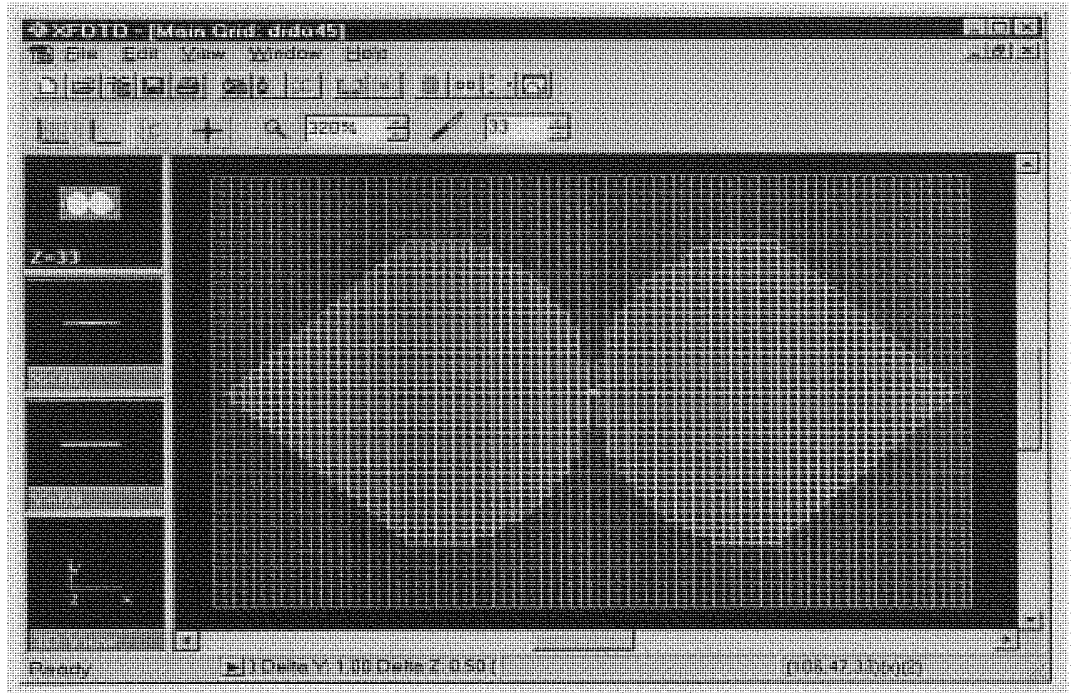

Fig. 30. Layer $\mathrm{z}=33$ (xy plane) of MDIDO-C1 in XFDTD software.

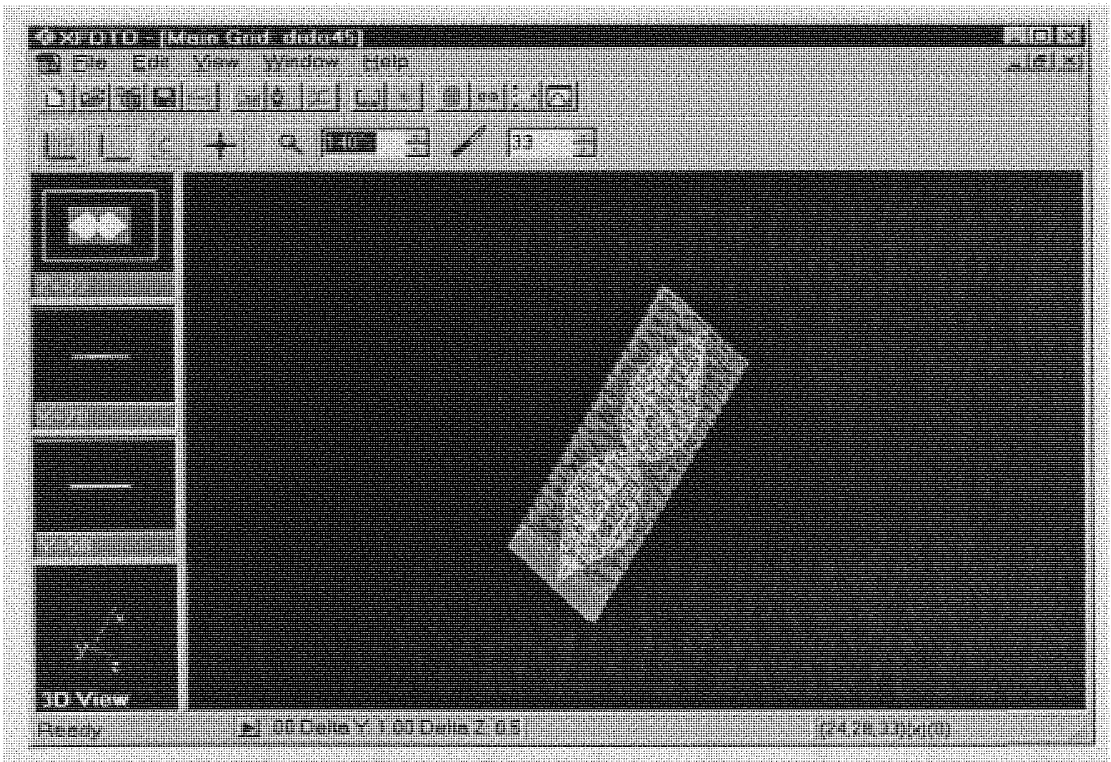

Fig. 31. 3-Dimensional view of MDIDO-C1 in XFDTD software. 
Fig. 32 and Fig. 33 show variation of return loss and input impedance with frequency respectively.

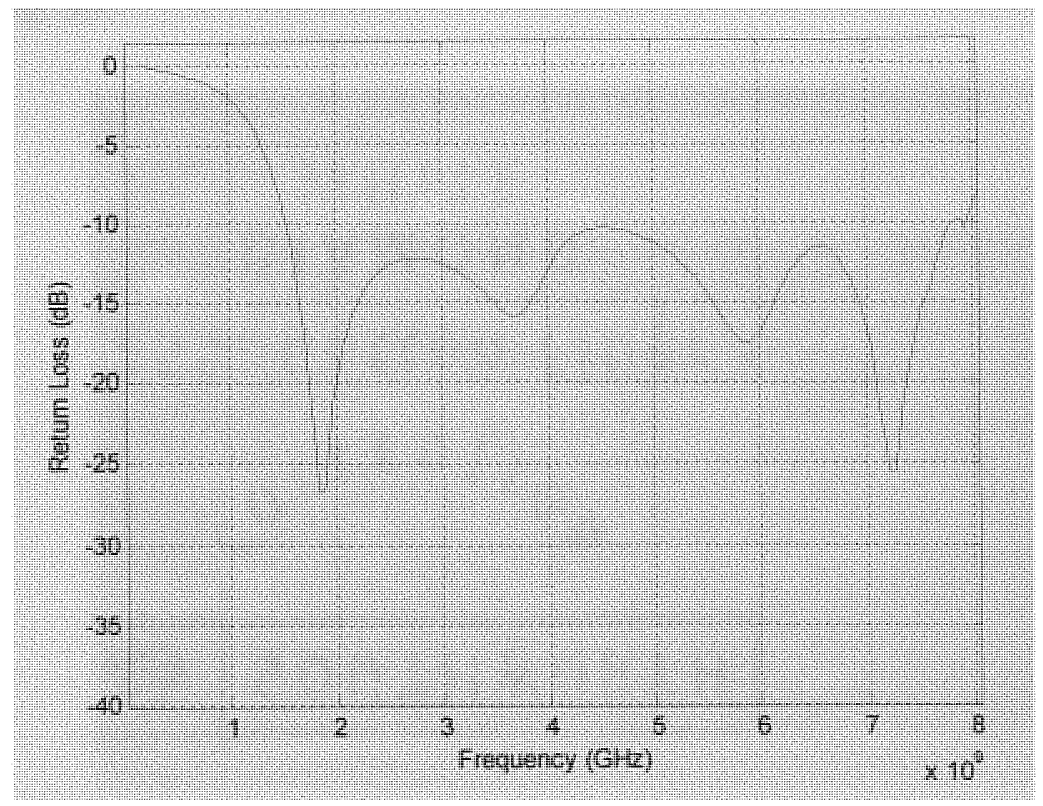

Fig. 32. Frequency Vs. return loss for MDIDO-C1.

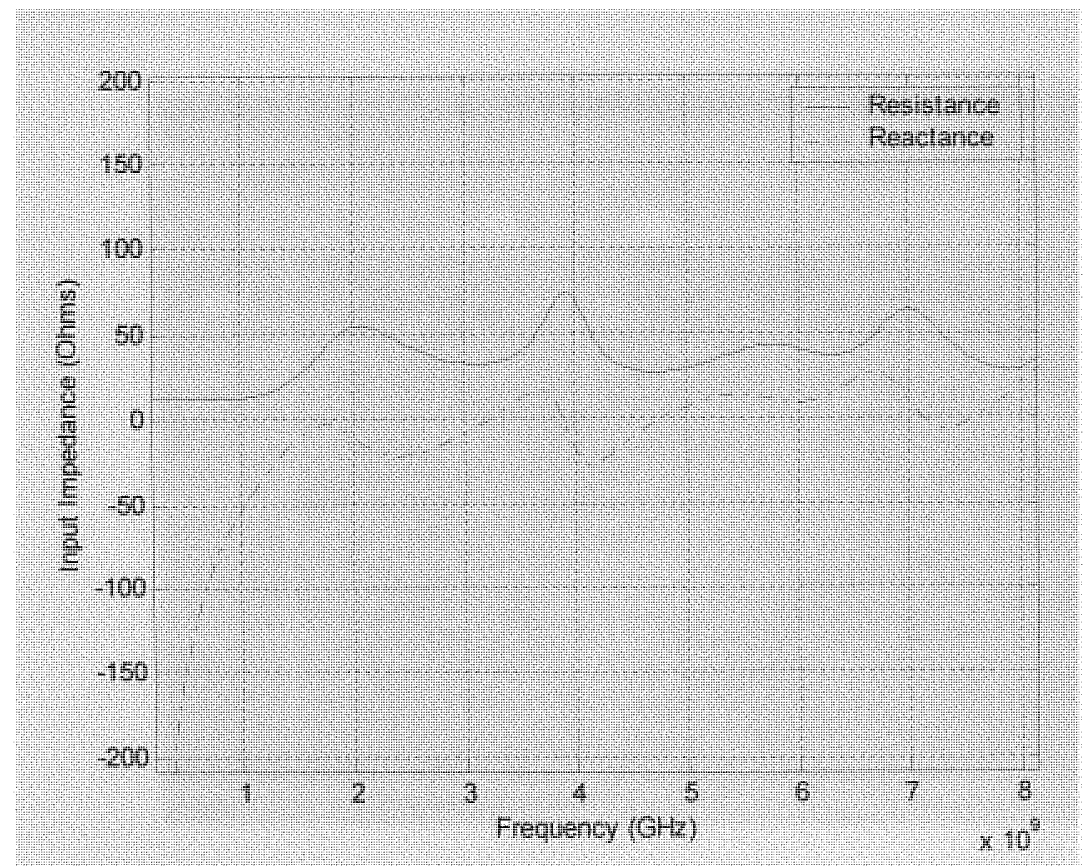

Fig. 33. Frequency Vs. input impedance for MDIDO-C1. 


\subsubsection{Modified diamond dipole, model MDIDO-A2}

MDIDO-A2 antenna is constructed in XFDTD software as shown in Fig. 34. A 3-D view is shown in Fig. 35.



Fig. 34. Layer $z=33$ (xy plane) of MDIDO-A2 in XFDTD software.

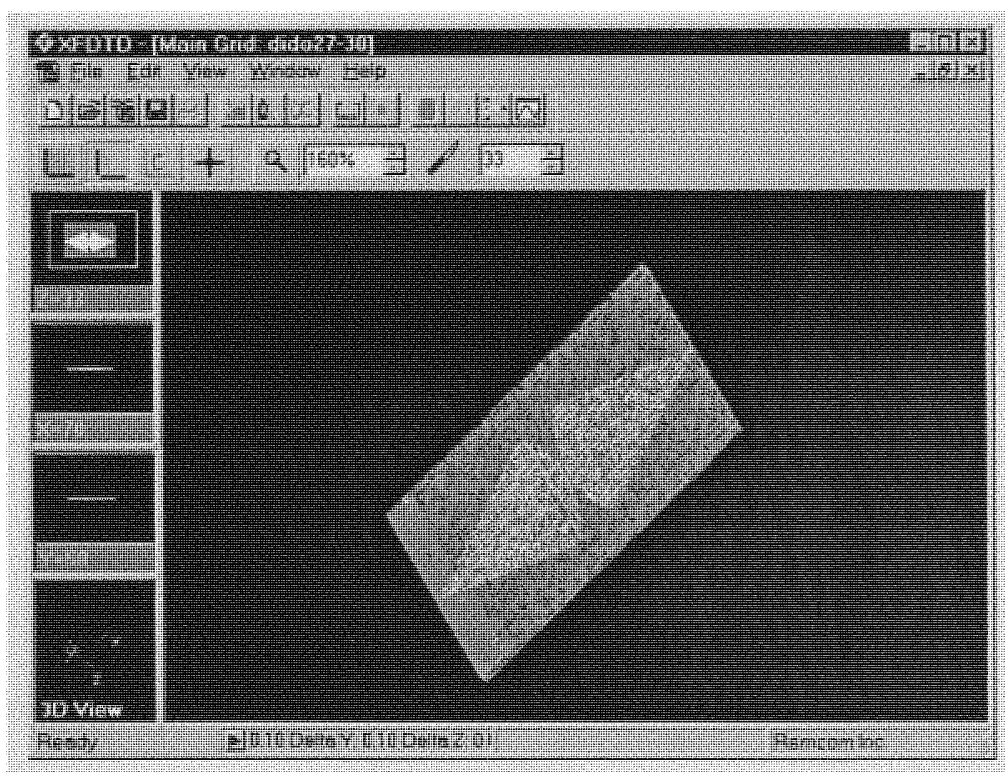

Fig. 35. 3-Dimensional view of MDIDO-A2 in XFDTD software. 
Fig. 36 and Fig. 37 show variation of return loss and input impedance with frequency respectively.

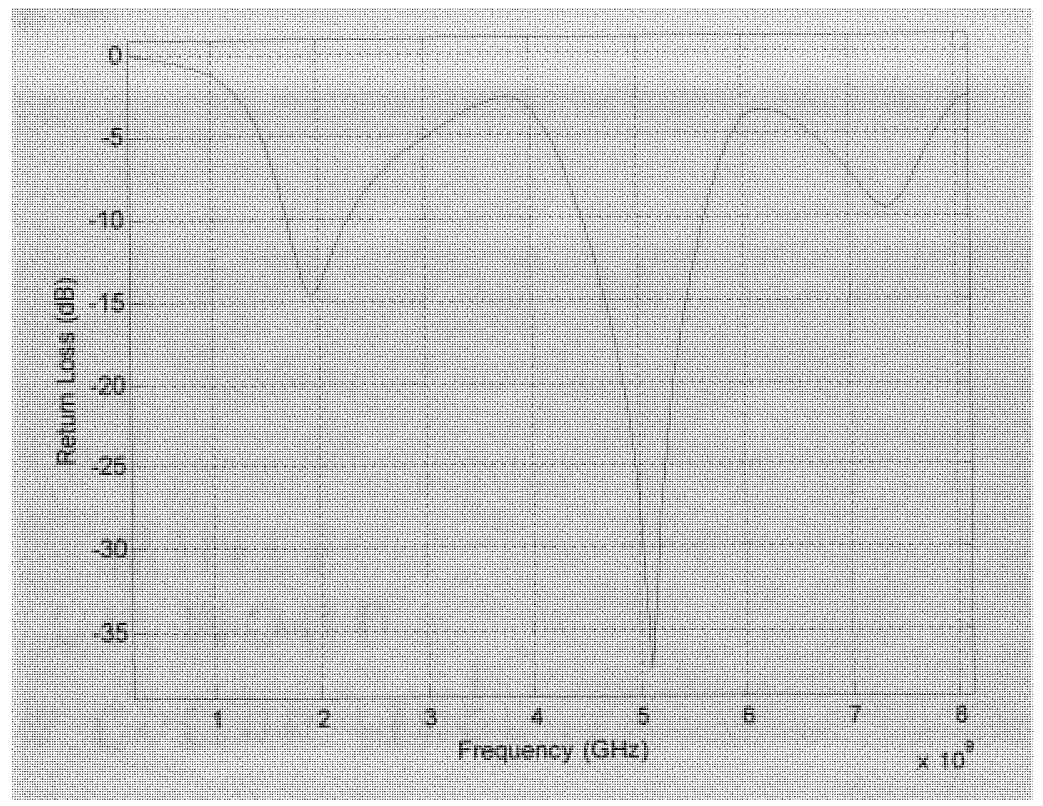

Fig. 36. Frequency Vs. return loss for MDIDO-A2.

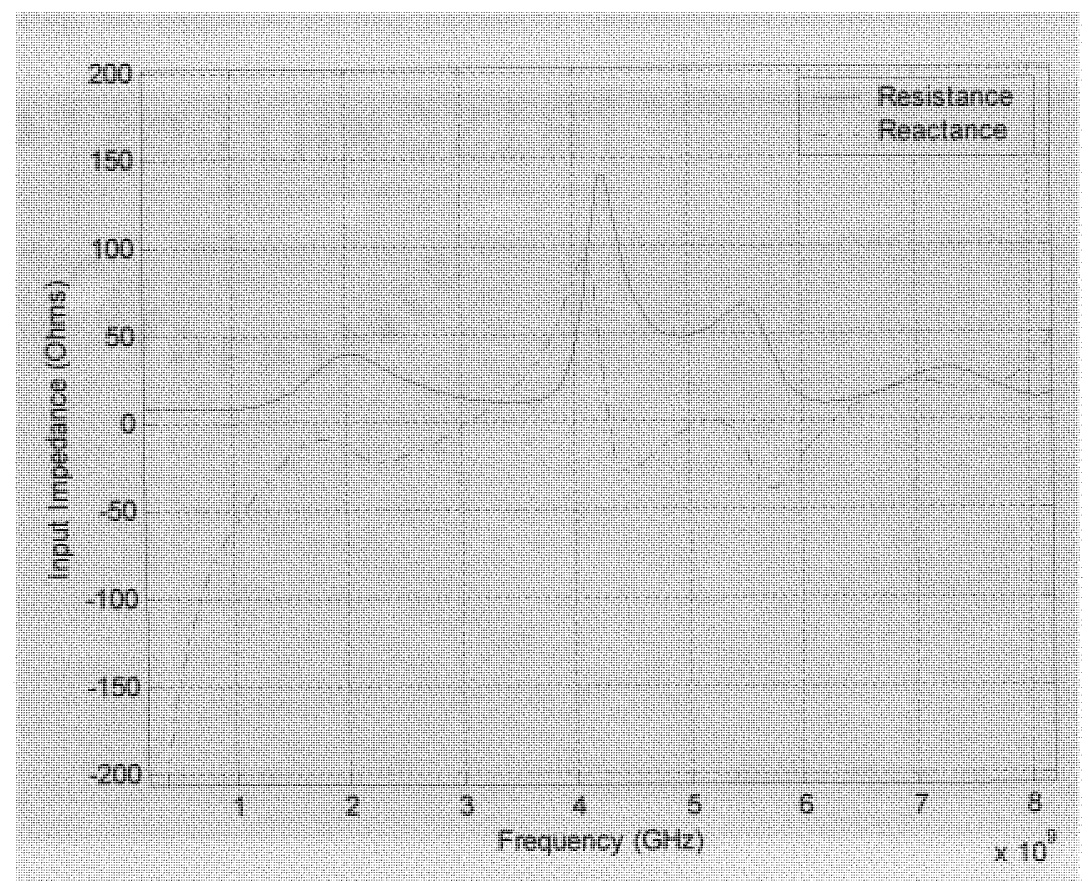

Fig. 37. Frequency Vs. input impedance for MDIDO-A2. 


\subsubsection{Modified diamond dipole, model MDIDO-B2}

MDIDO-C1 antenna is constructed in XFDTD software as shown in Fig. 38. A 3-D view is shown in Fig. 39.

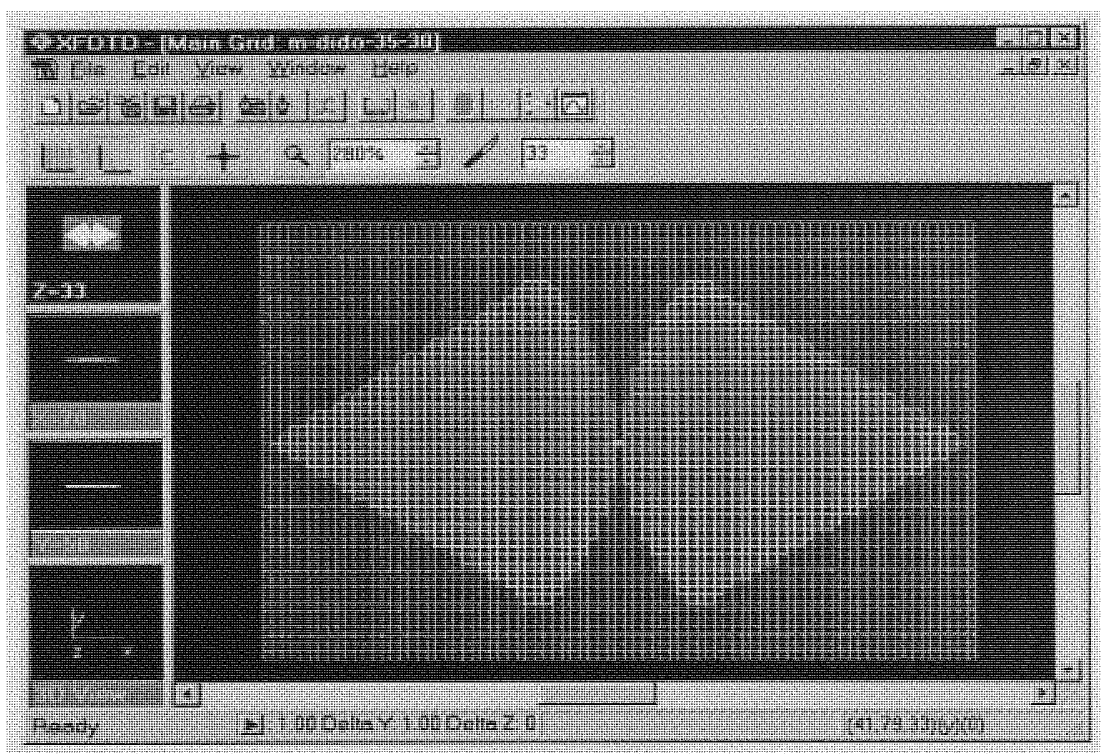

Fig. 38. Layer $\mathrm{z}=33$ (xy plane) of MDIDO-B2 in XFDTD software.

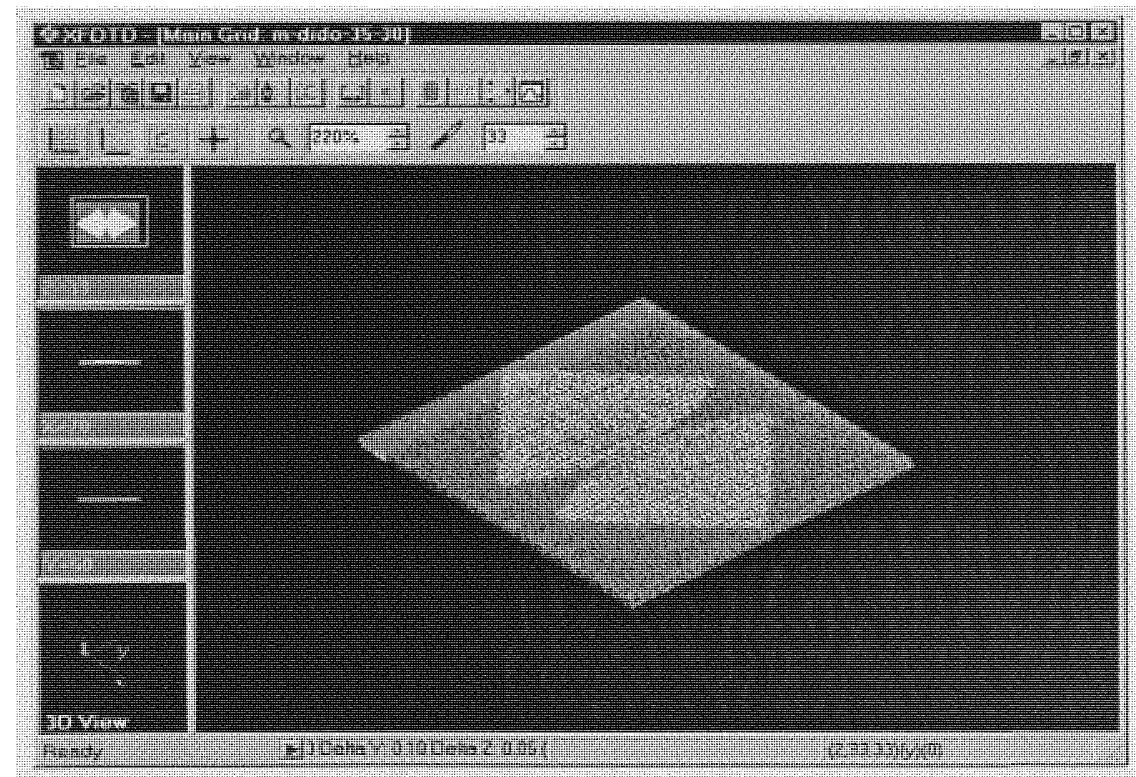

Fig. 39. 3-Dimensional view of MDIDO-B2 in XFDTD software. 
Fig. 40 and Fig. 41 show variation of return loss and input impedance with frequency respectively.

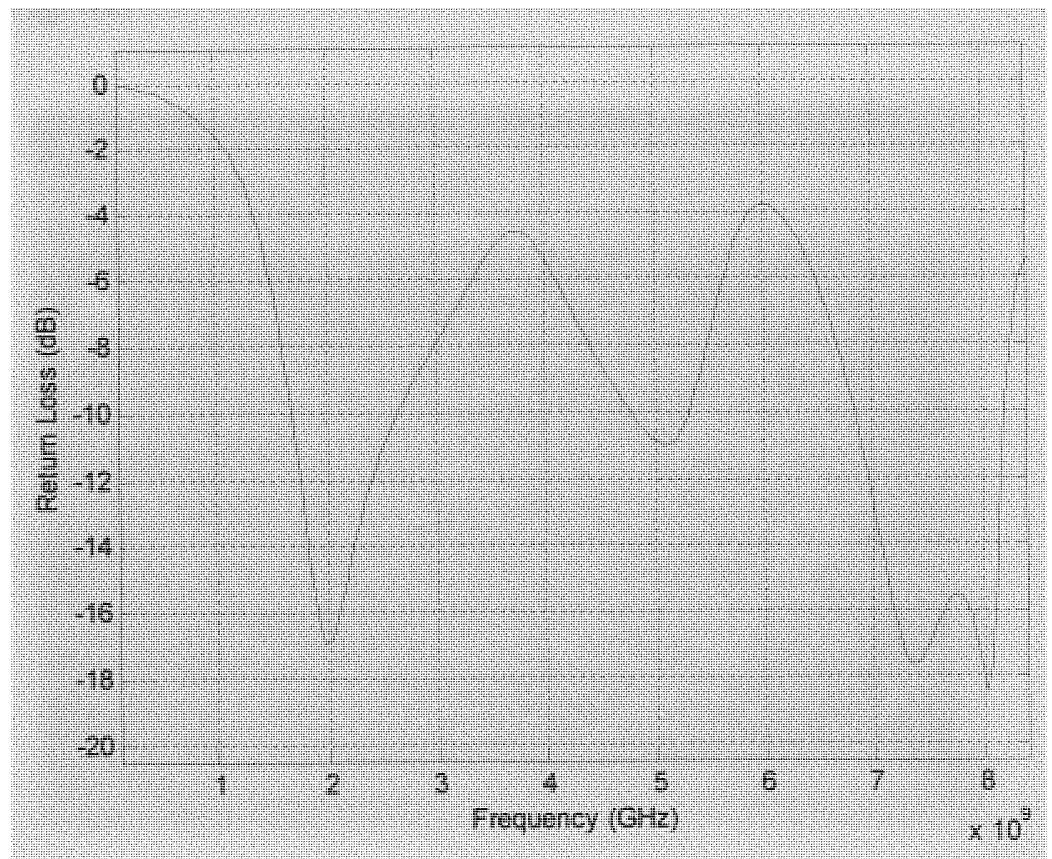

Fig. 40. Frequency Vs. return loss for MDIDO-B2.

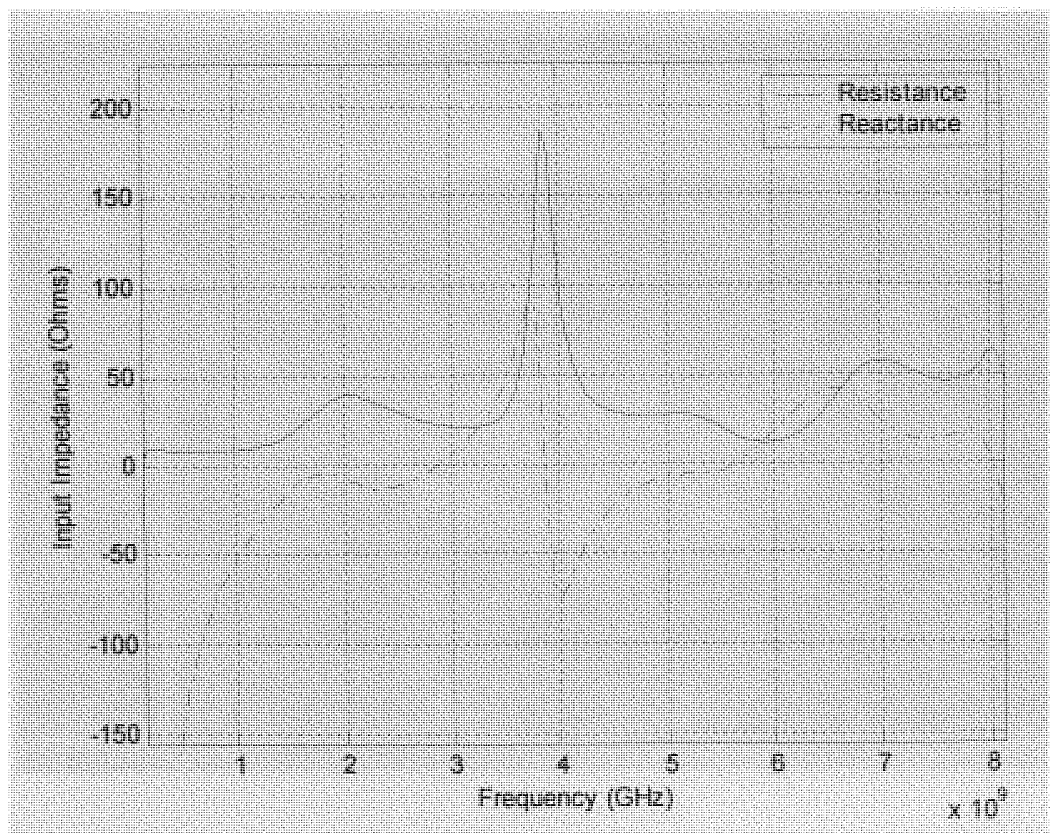

Fig. 41. Frequency Vs. input impedance for MDIDO-B2. 


\subsubsection{Modified diamond dipole, model MDIDO-C2}

MDIDO-A2 antenna is constructed in XFDTD software as shown in Fig. 42. A 3-D view is shown in Fig. 43.

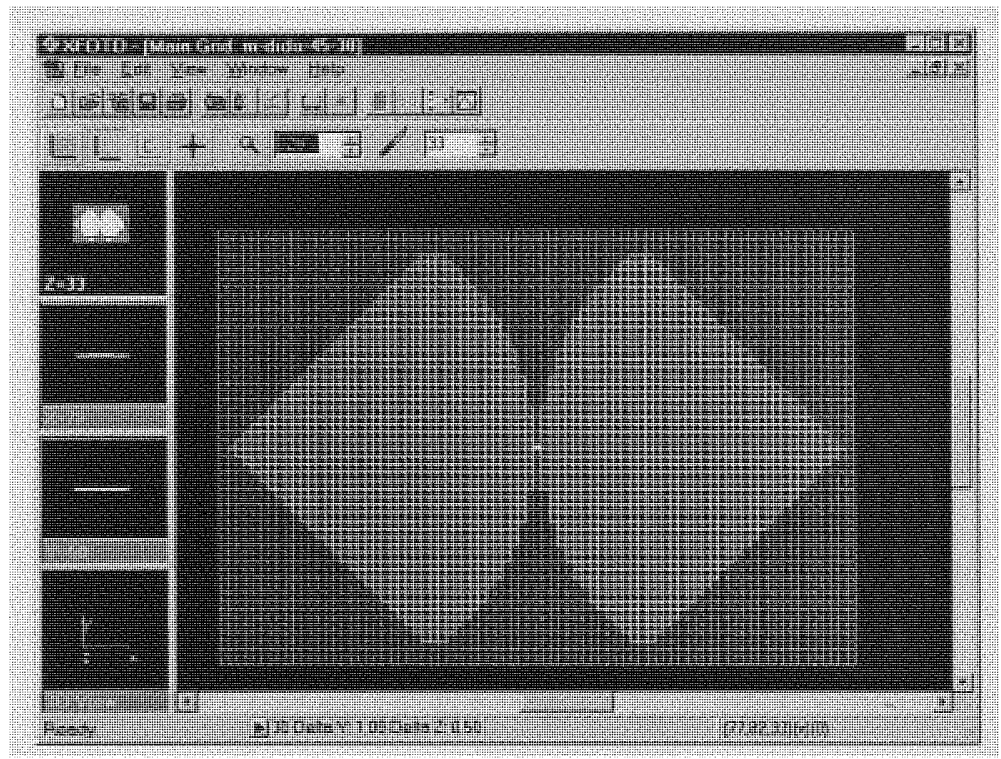

Fig. 42. Layer $\mathrm{z}=33$ (xy plane) of MDIDO-C2 in XFDTD software.

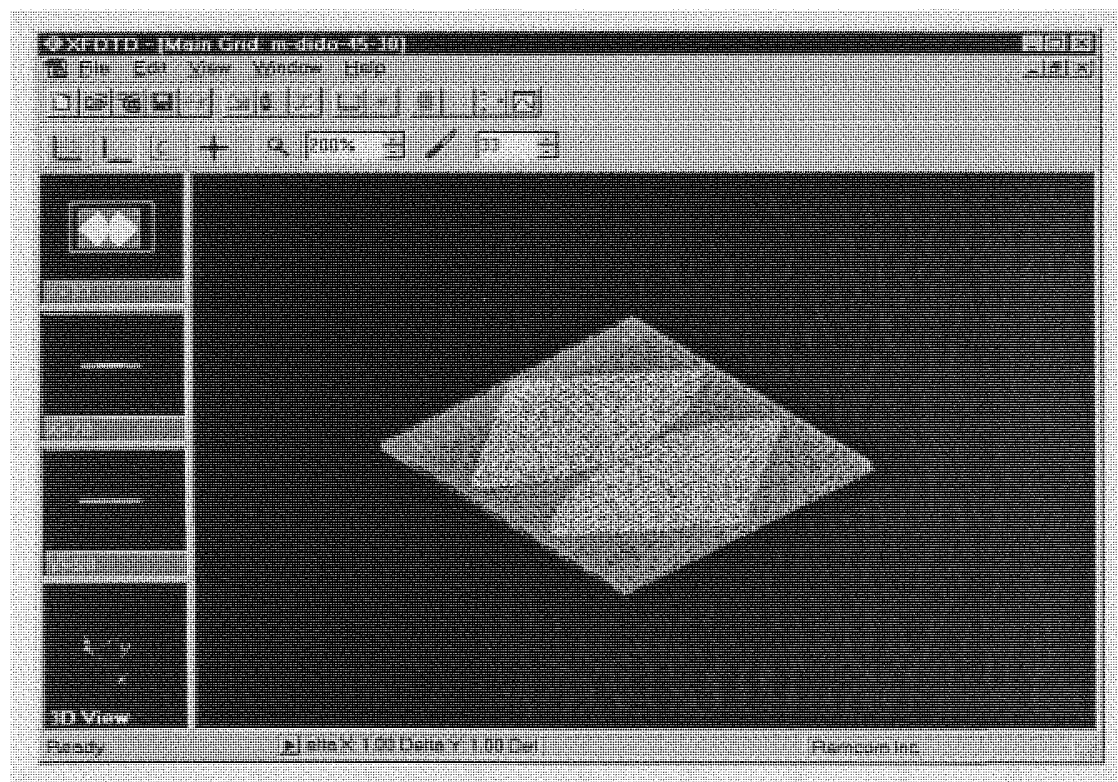

Fig. 43. 3-Dimensional view of MDIDO-C2 in XFDTD software. 
Fig. 44 and Fig. 45 show variation of return loss and input impedance with frequency respectively.

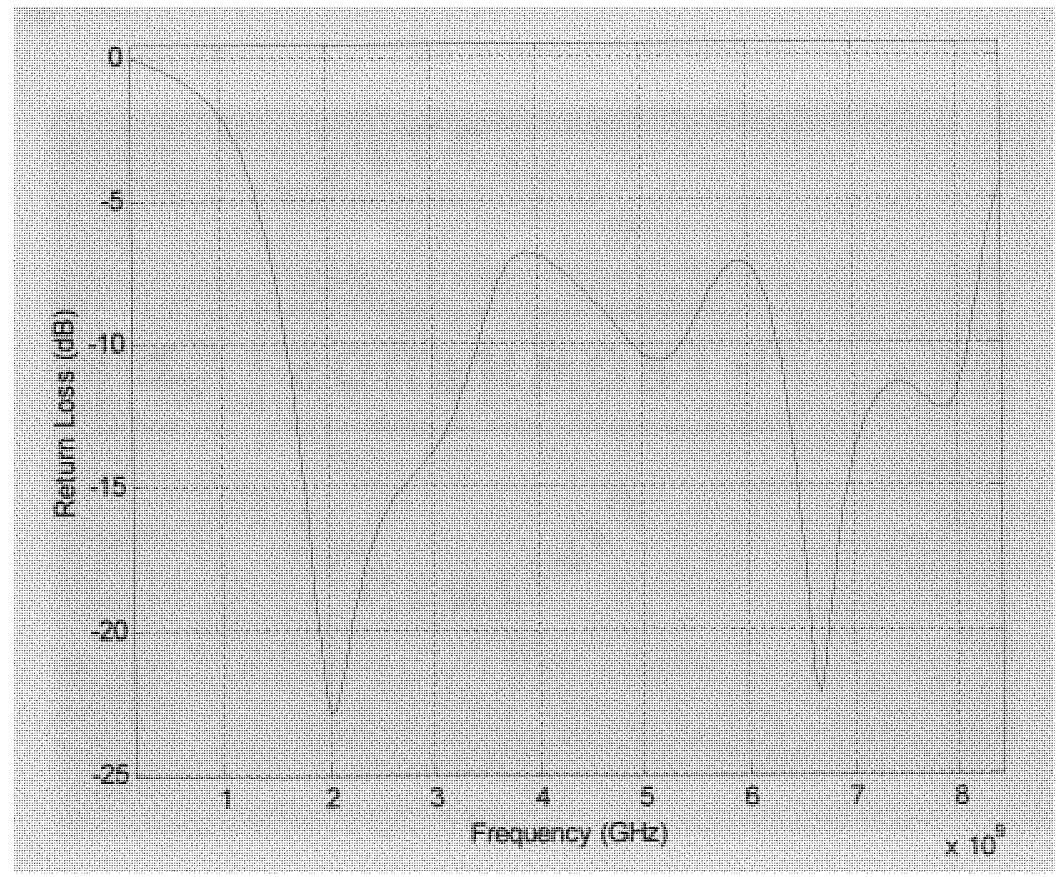

Fig. 44. Frequency Vs. return loss for MDIDO-C2.

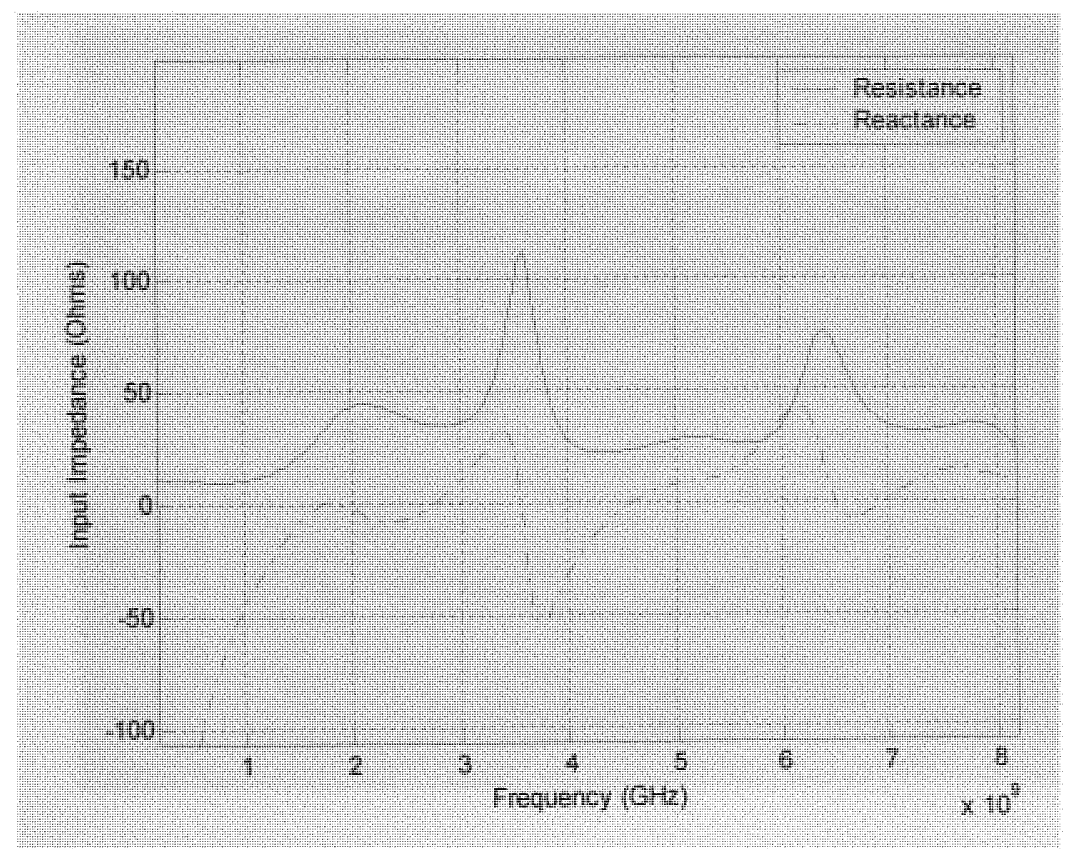

Fig. 45. Frequency Vs. input impedance for MDIDO-C2. 


\subsubsection{Modified diamond dipole, model MDIDO-A3}

MDIDO-A3 antenna is constructed in XFDTD software as shown in Fig. 46. A 3-D view is shown in Fig. 47.

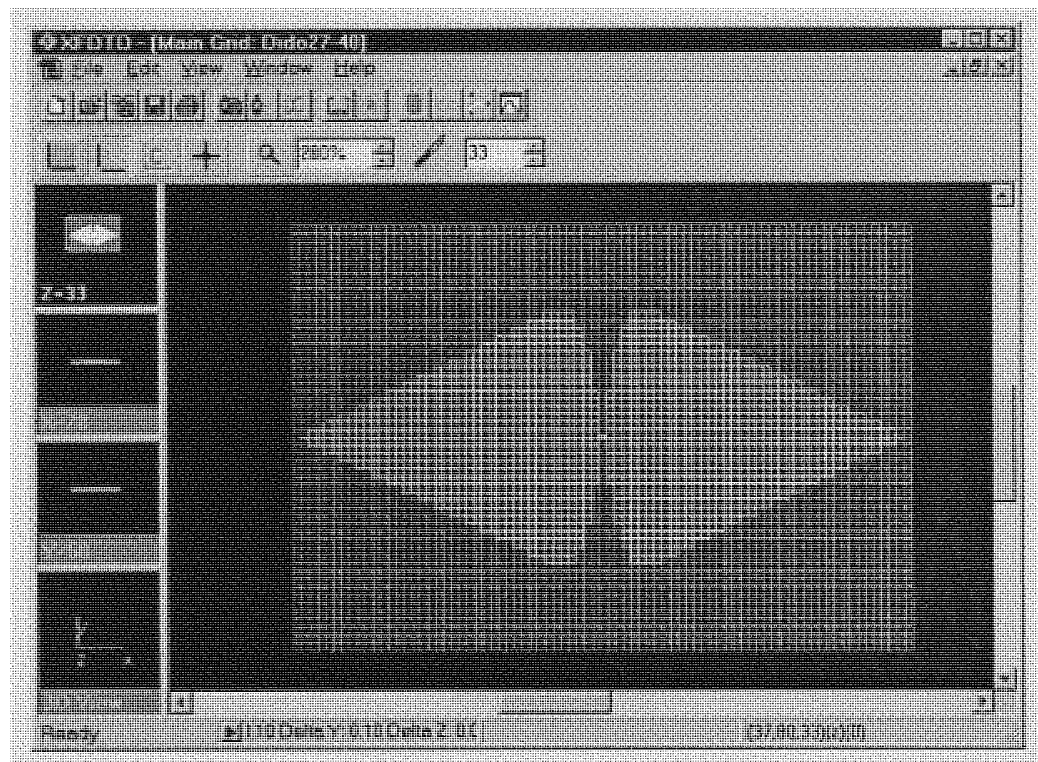

Fig. 46. Layer $\mathrm{z}=33$ (xy plane) of MDIDO-A3 in XFDTD software.

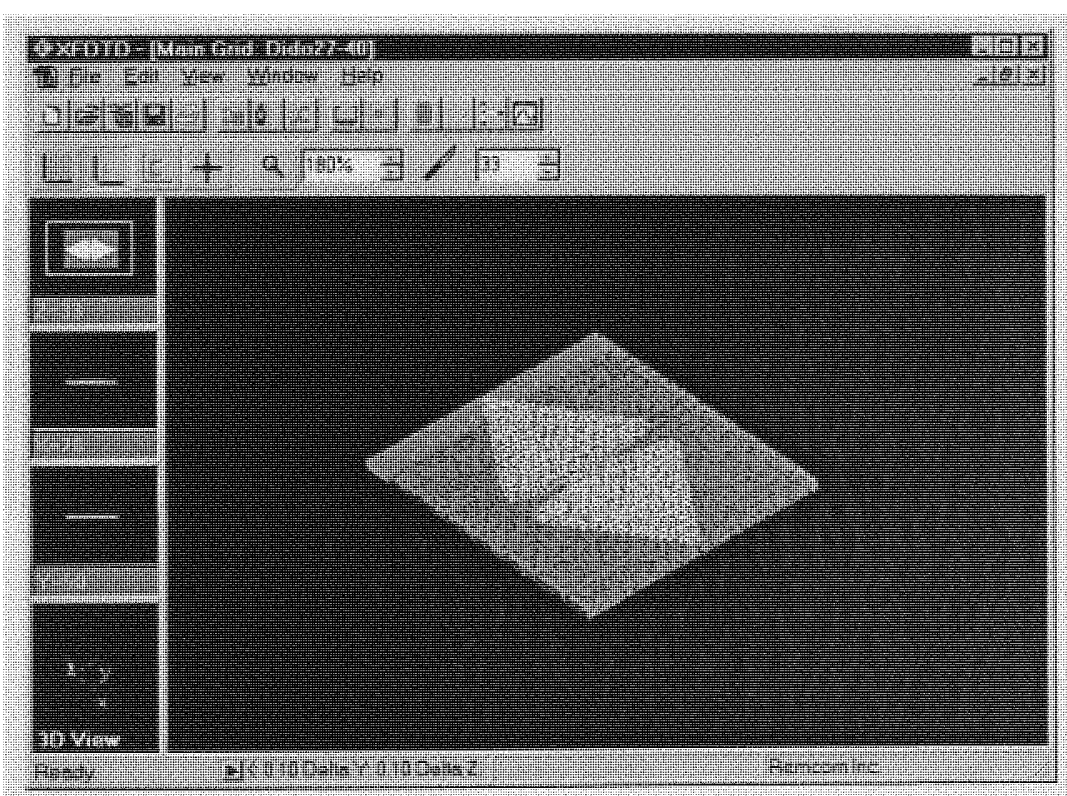

Fig. 47. 3-Dimensional view of MDIDO-A3 in XFDTD software. 
Fig. 48 and Fig. 49 show variation of return loss and input impedance with frequency respectively.

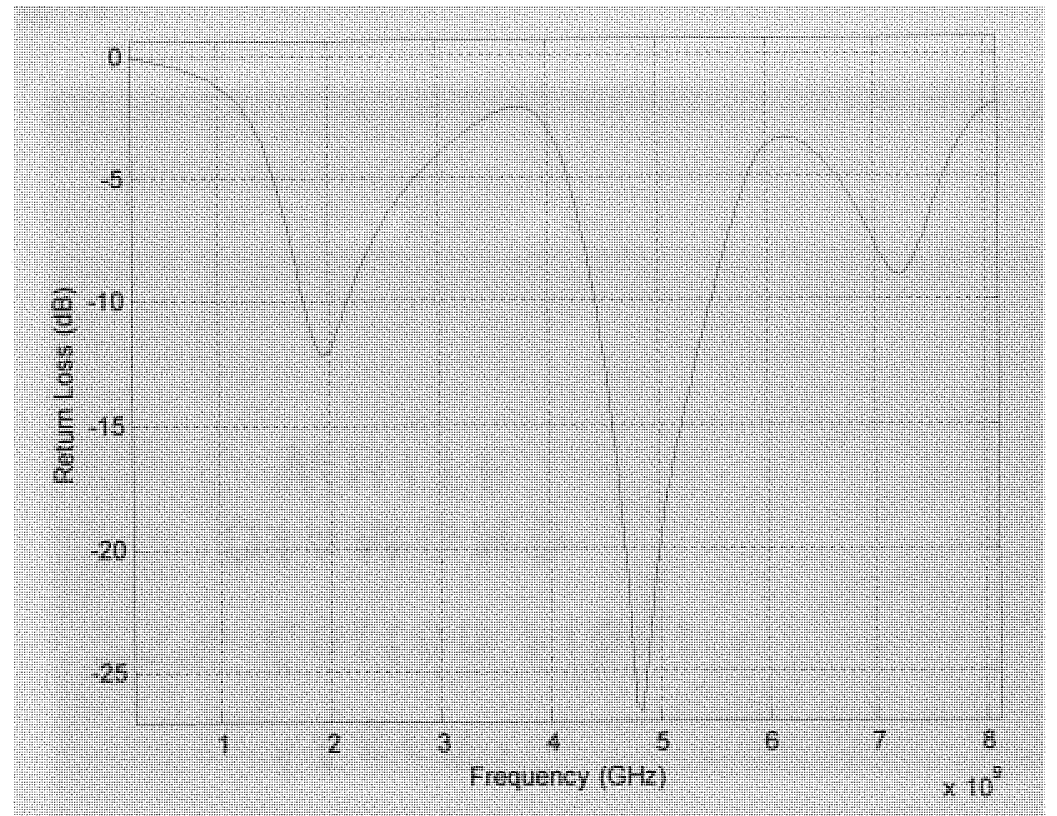

Fig. 48. Frequency Vs. return loss for MDIDO-A3.

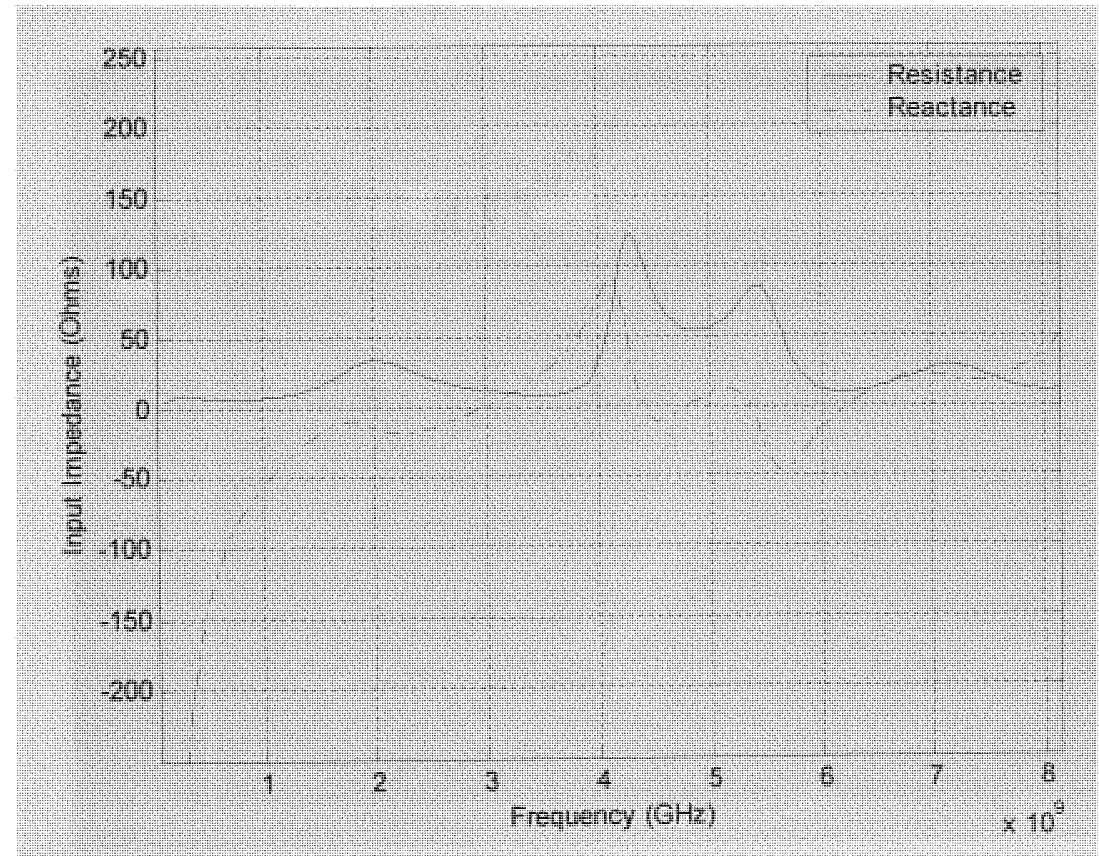

Fig. 49. Frequency Vs. input impedance for MDIDO-A3. 


\subsubsection{Modified diamond dipole, model MDIDO-B3}

MDIDO-B3 antenna is constructed in XFDTD software as shown in Fig. 50. A 3-D view is shown in Fig. 51.

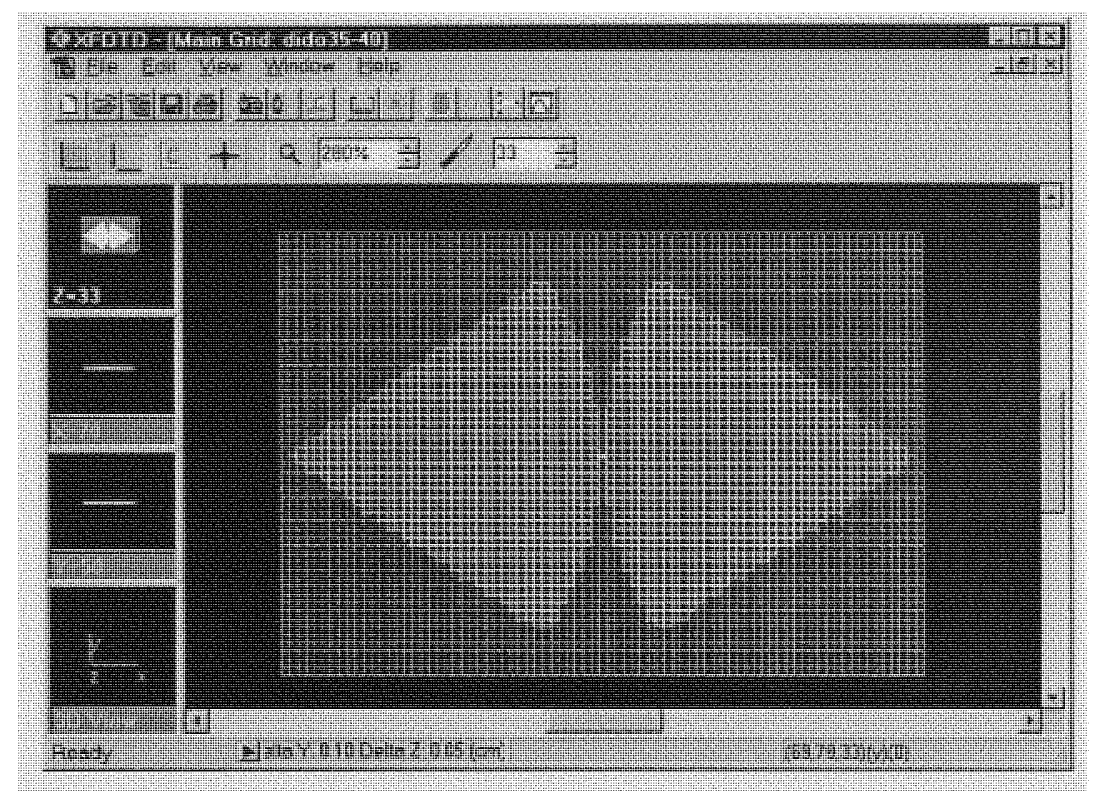

Fig. 50. Layer $\mathrm{z}=33$ (xy plane) of MDIDO-B3 in XFDTD software.

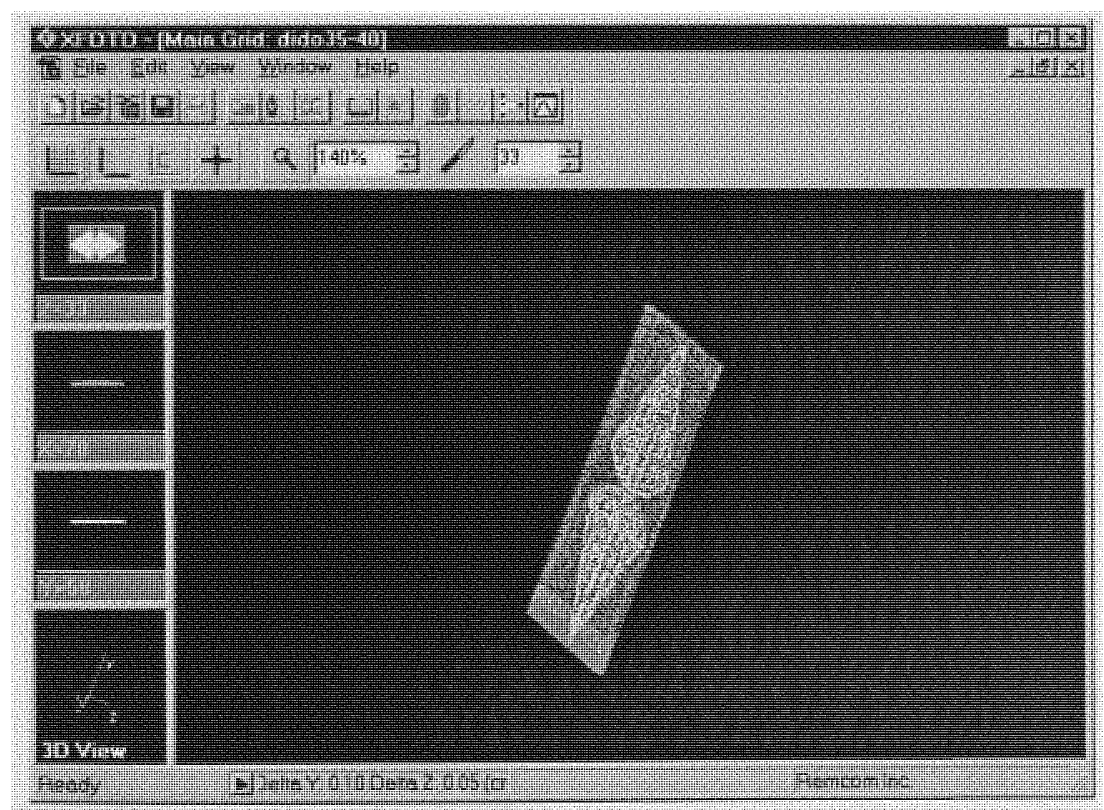

Fig. 51. 3-Dimensional view of MDIDO-B3 in XFDTD software. 
Fig. 52 and Fig. 53 show variation of return loss and input impedance with frequency respectively.

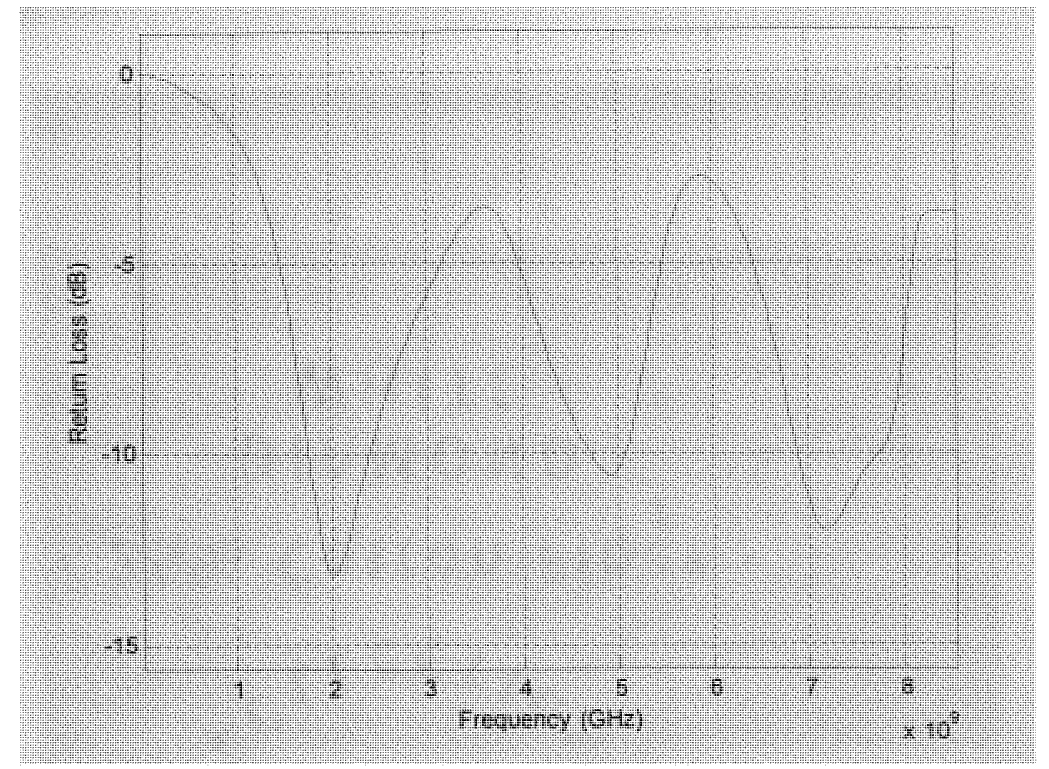

Fig. 52. Frequency Vs. return loss for MDIDO-B3.

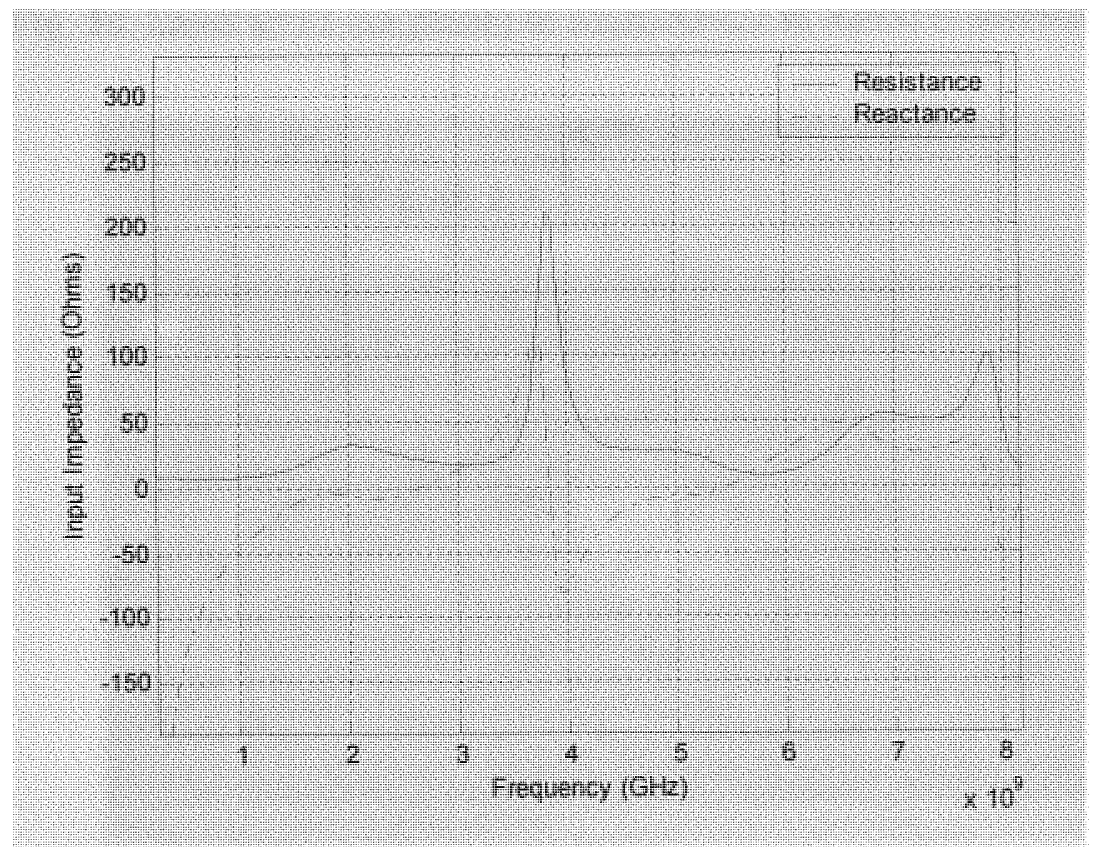

Fig. 53. Frequency Vs. input impedance for MDIDO-B3. 


\subsubsection{Modified diamond dipole, model MDIDO-C3}

MDIDO-C3 antenna is constructed in XFDTD software as shown in Fig. 54. A 3-D view is shown in Fig. 55.

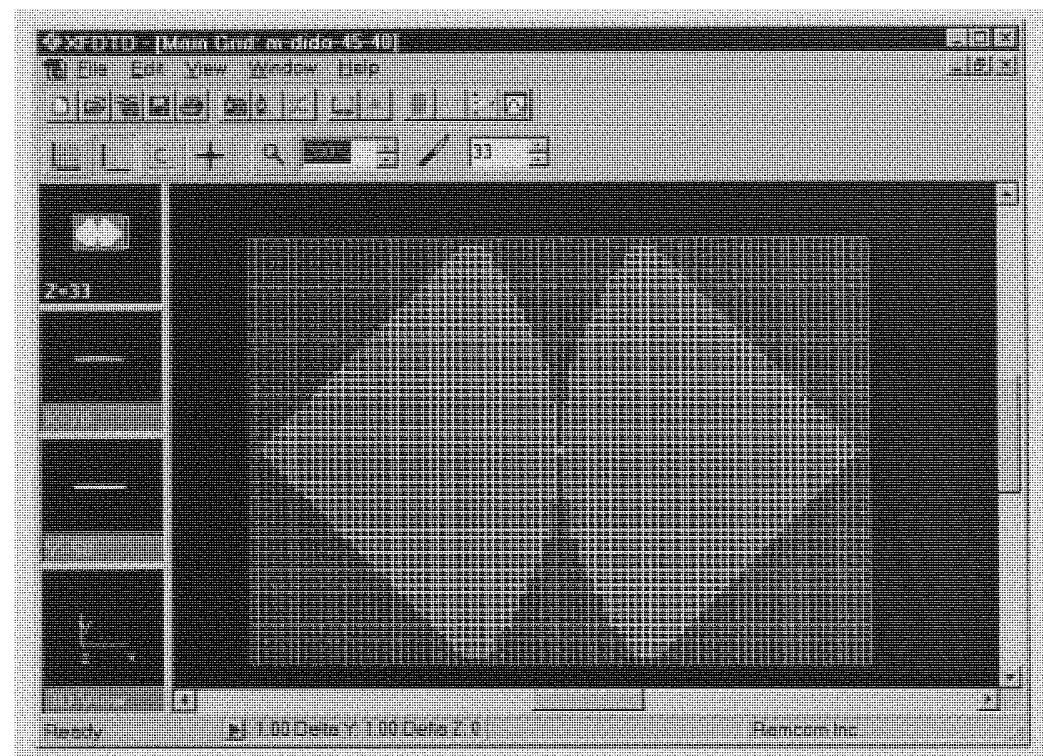

Fig. 54. Layer $z=33$ (xy plane) of MDIDO-C3 in XFDTD software.

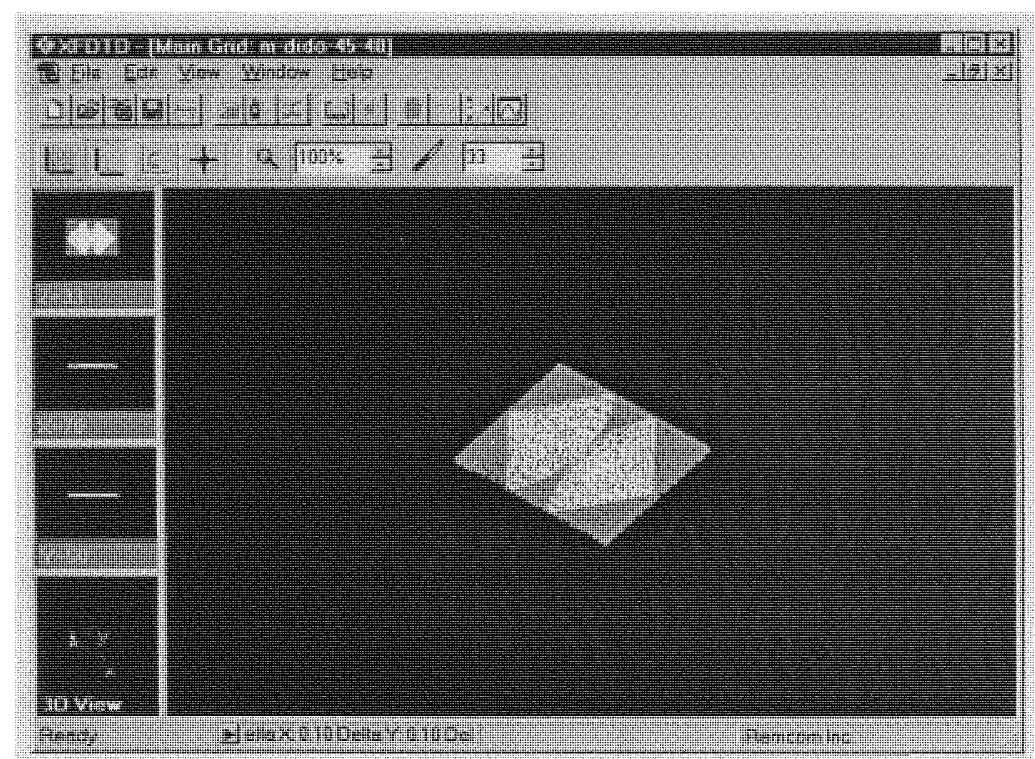

Fig. 55. 3-Dimensional view of MDIDO-C3 in XFDTD software. 
Fig. 56 and Fig. 57 show variation of return loss and input impedance with frequency respectively.

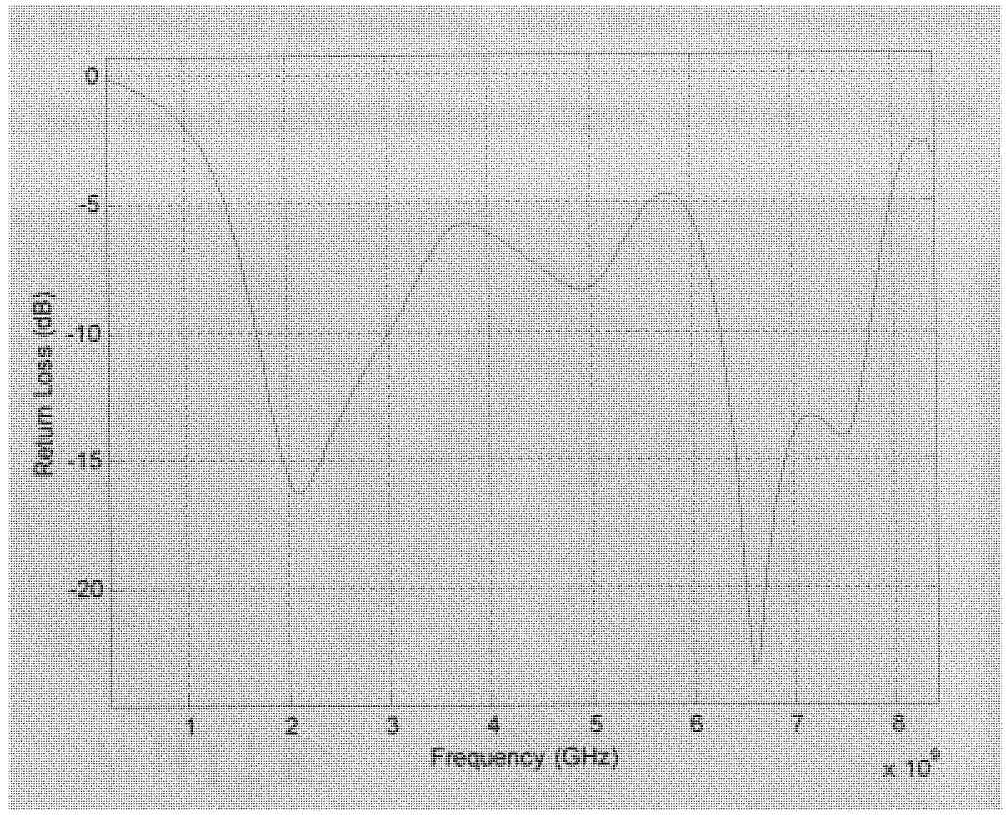

Fig. 56. Frequency Vs. return ross for MDIDO-C3.

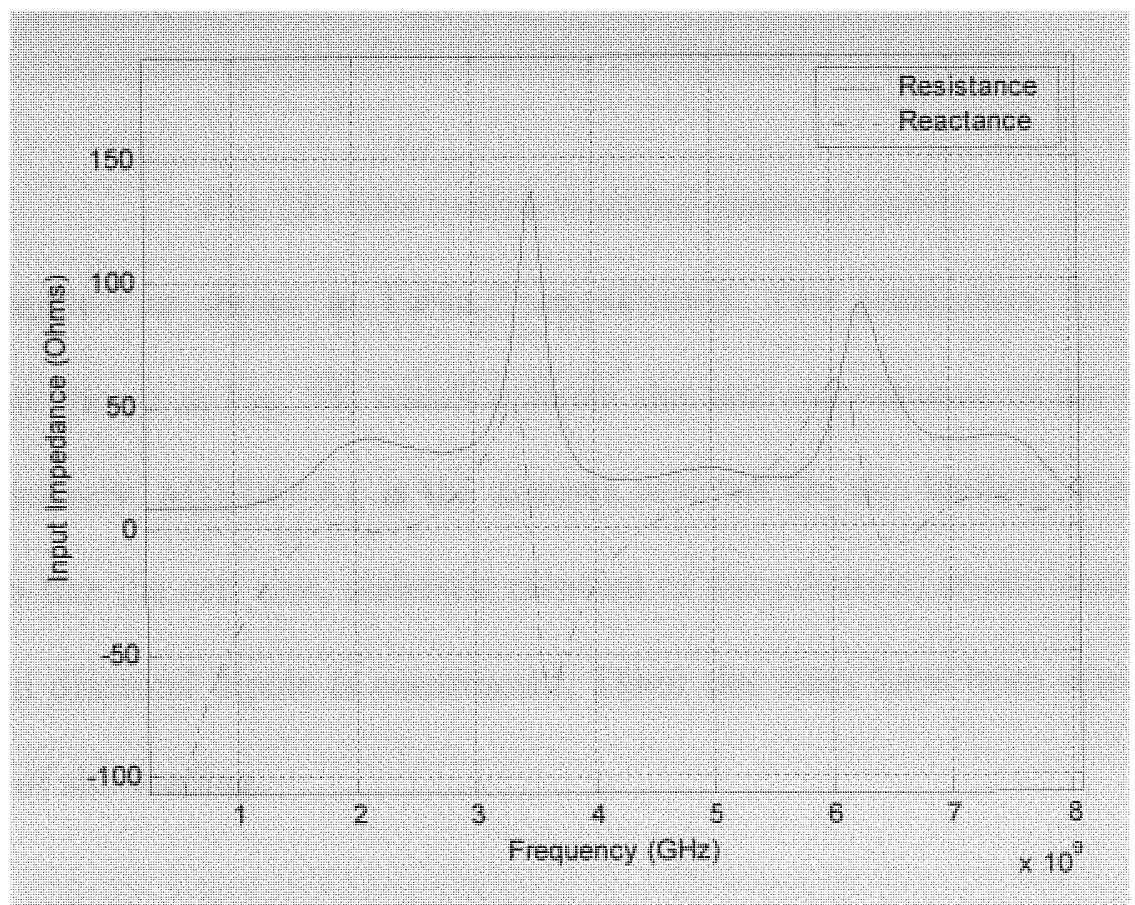

Fig. 57. Frequency Vs. input impedance for MDIDO-C3. 


\subsection{Part III Slot loaded modified diamond dipole}

\subsubsection{Geometry}

To study the effect of slot loading, slots have been incorporated into modified diamond dipole. Slot loaded modified diamond dipole is shown in Fig. 58.

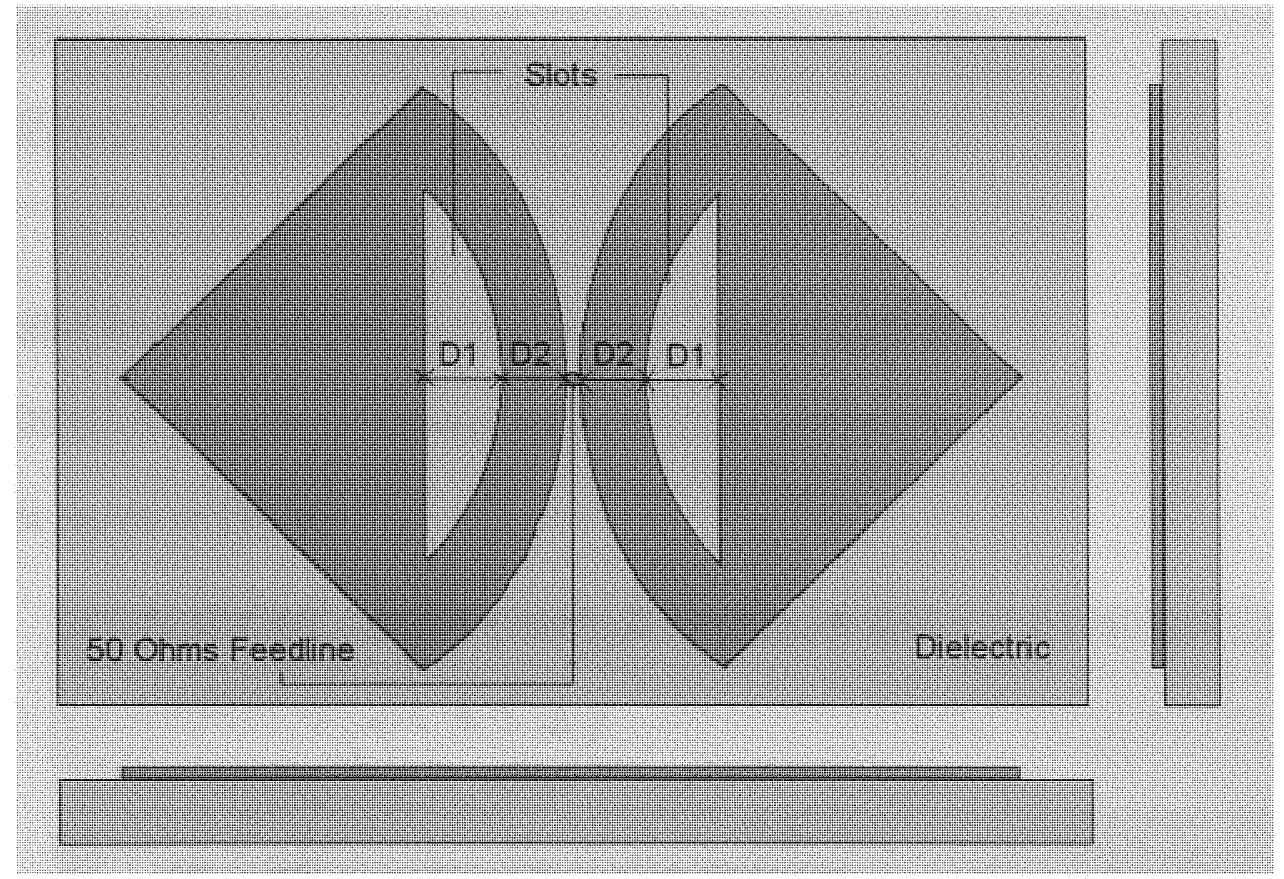

Fig. 58. Slot loaded modified diamond dipole.

Antenna models as shown in Table 2 are selected. Two parameters, D1 and D2 are selected (as shown in Fig. 58) which indicate slot size and distance of the slot from the feed respectively. MDIDO-A1 is the antenna selected for slot loading. Keeping all the parameters like the cell size, dielectric constant of the substrate, source details constant, slot loaded diamond dipole is constructed. Three slot sizes have been selected to study the effect of slot loading. 
Information about the slot loaded modified diamond dipole antenna models

\begin{tabular}{|c|c|c|}
\hline Antenna Model & Slot width (D1 mm) & Distance of slot from feed (D2 mm) \\
\hline SLMDIDO-A & 8 & 1 \\
\hline SLMDIDO-B & 6 & 3 \\
\hline SLMDIDO-C & 4 & 5 \\
\hline
\end{tabular}

\subsubsection{Slot loaded modified diamond dipole, model SLMDIDO-A}

SLMDIDO-A antenna is constructed in XFDTD software as shown in Fig. 59. A 3-D view is shown in Fig. 60.

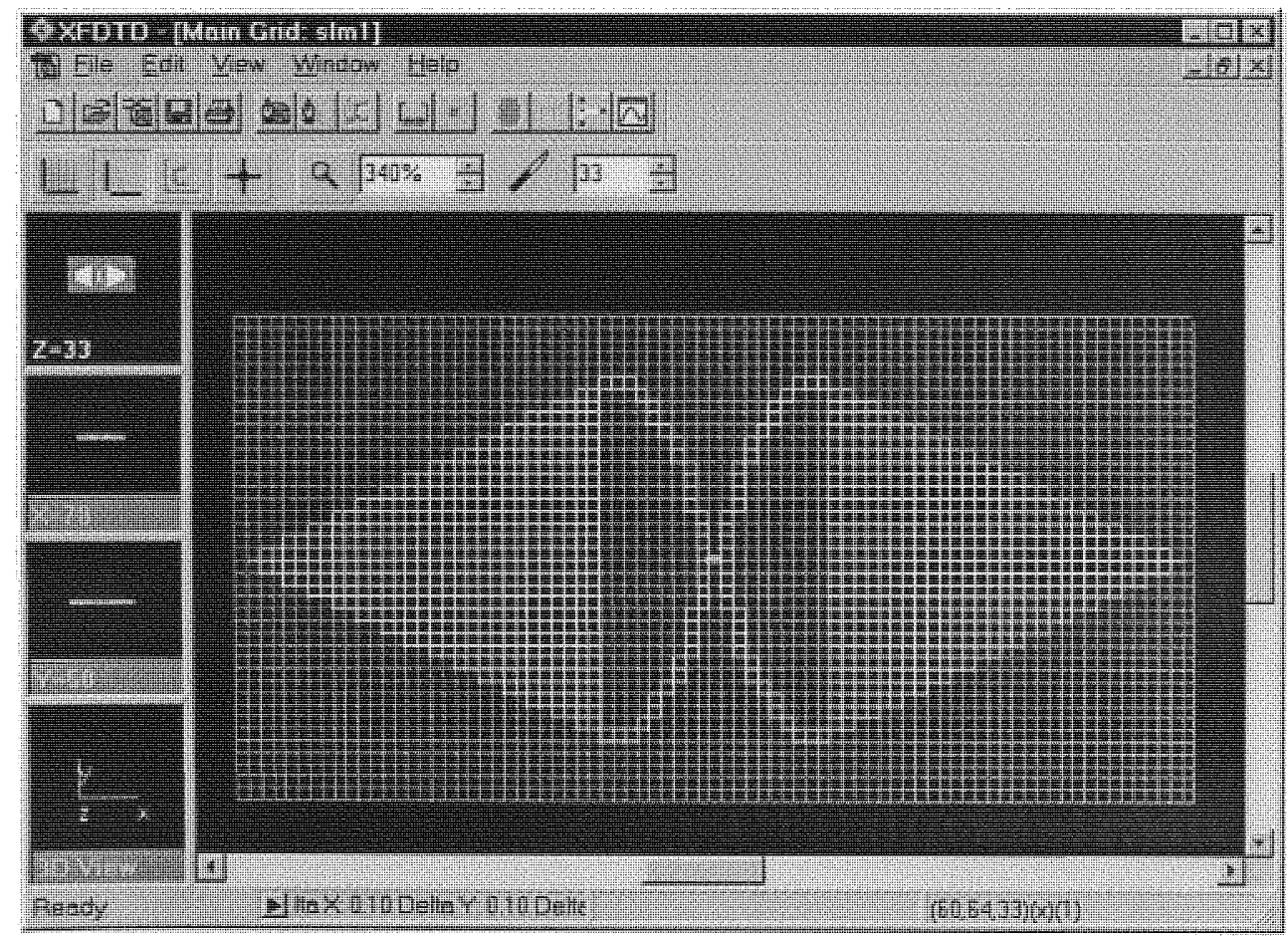

Fig. 59. Layer $z=33$ (xy plane) of SLMDIDO-A in XFDTD software. 


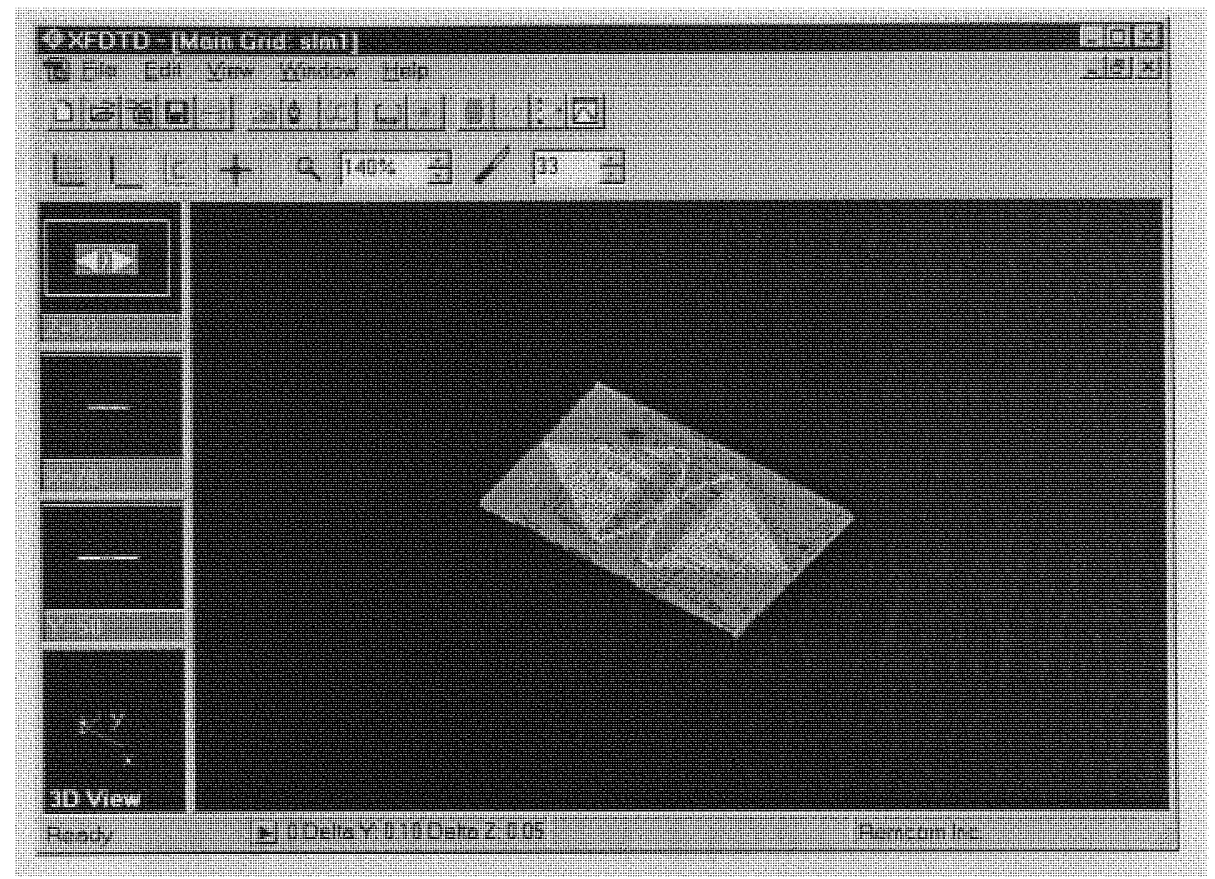

Fig. 60. 3-Dimensional view of SLMDIDO-A in XFDTD software.

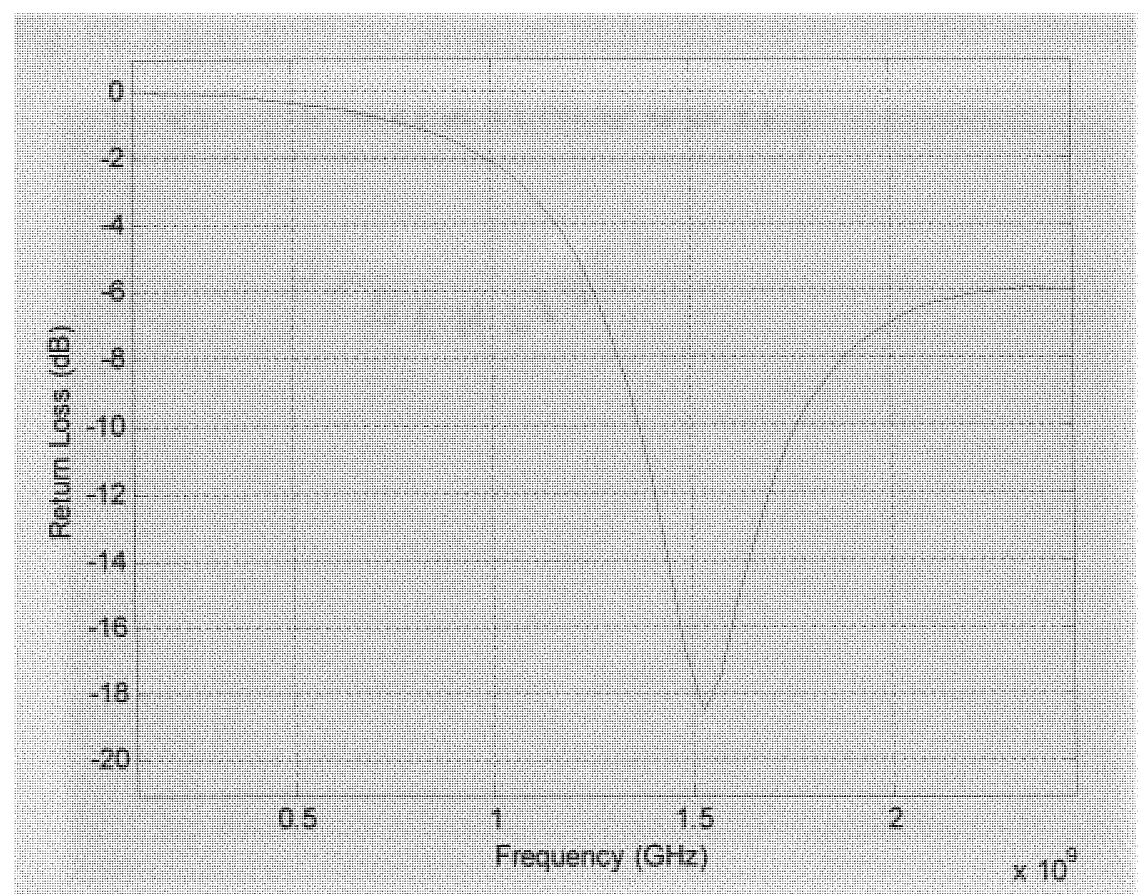

Fig. 61. Frequency Vs. return loss for SLMDIDO-A. 


\subsubsection{Slot loaded modified diamond dipole, model SLMDIDO-B}

SLMDIDO-A antenna is constructed in XFDTD software as shown in Fig. 62. A 3-D view is shown in Fig. 63.

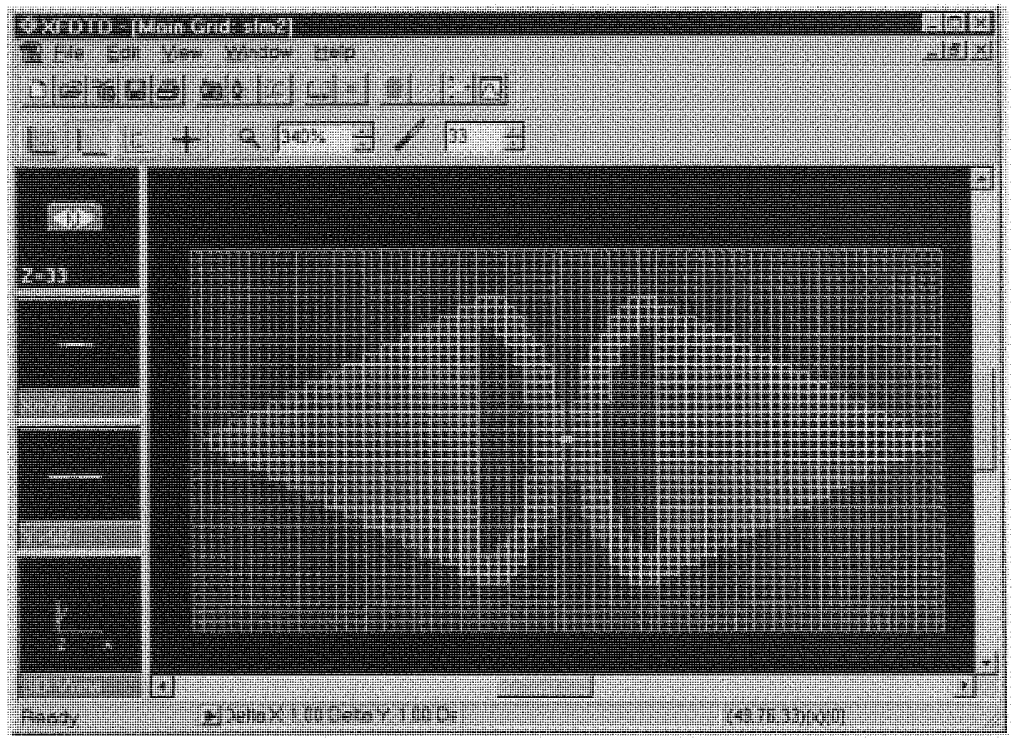

Fig. 62. Layer $\mathrm{z}=33$ (xy plane) of SLMDIDO-B in XFDTD software.

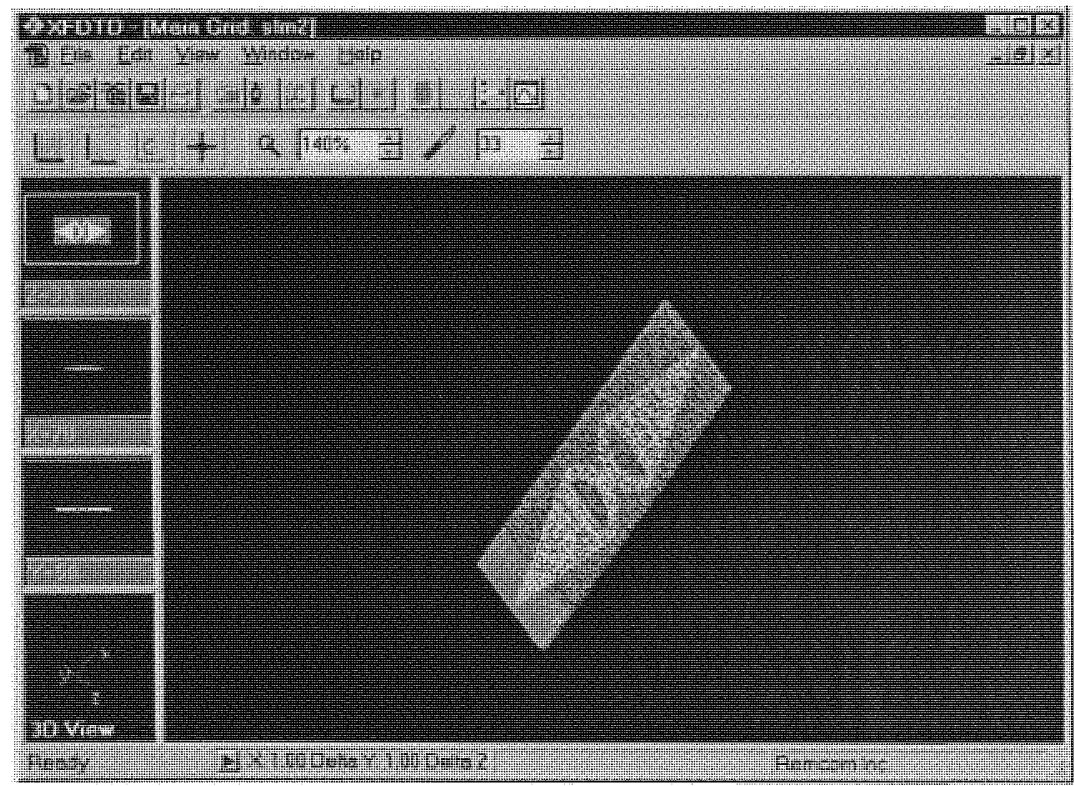

Fig. 63. 3-Dimensional view of SLMDIDO-B in XFDTD software. 


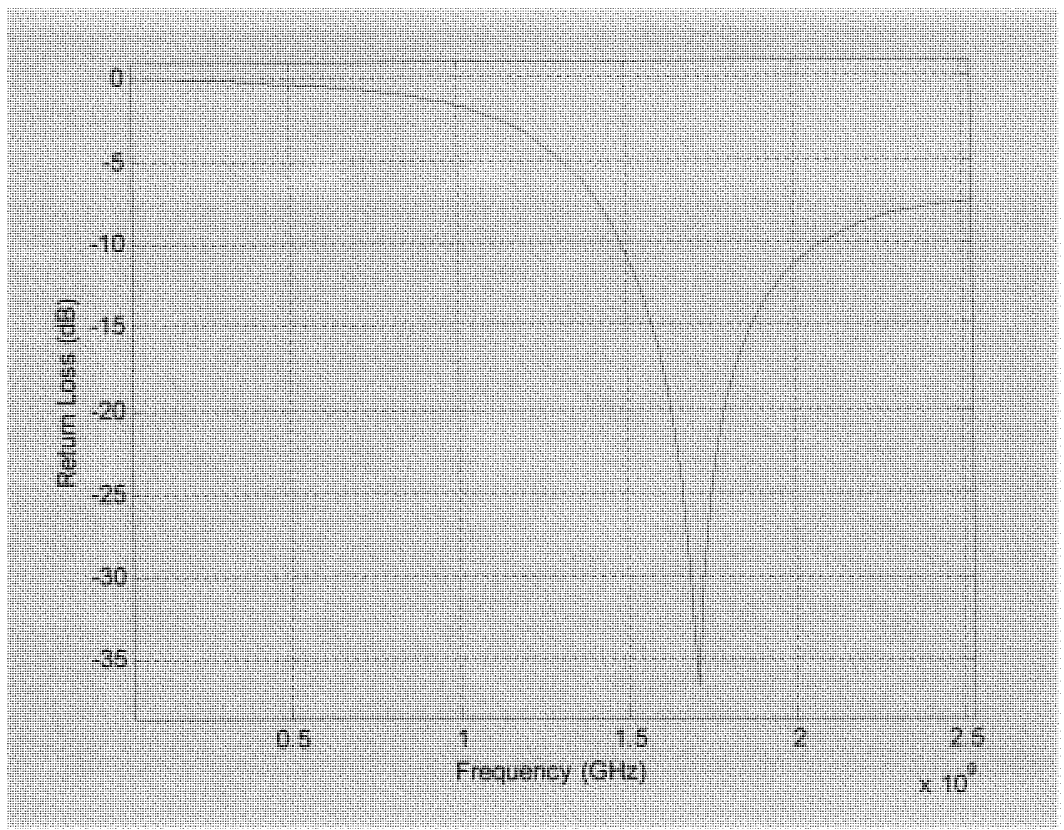

Fig. 64. Frequency Vs. return loss for SLMDIDO-B.

\subsubsection{Slot loaded modified diamond dipole, model SLMDIDO-C}

SLMDIDO-A antenna is constructed in XFDTD software as shown in Fig. 65. A 3-D view is shown in Fig. 66.

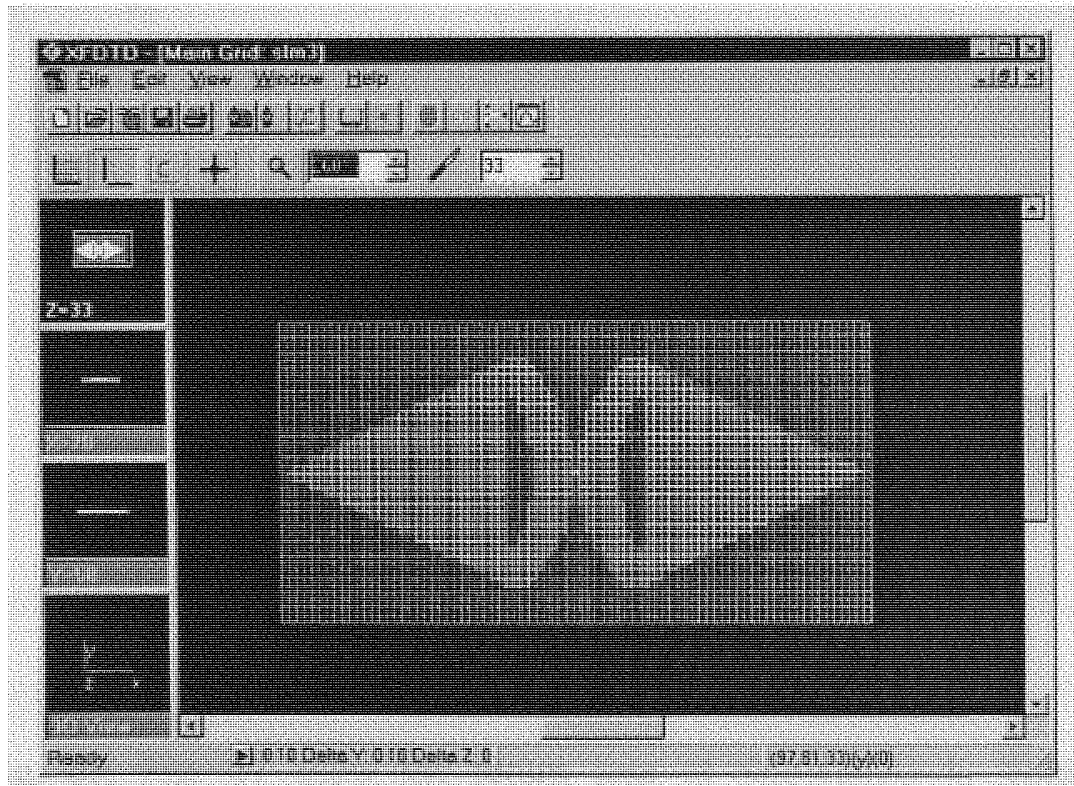

Fig. 65. Layer $z=33$ (xy plane) of SLMDIDO-C in XFDTD software. 


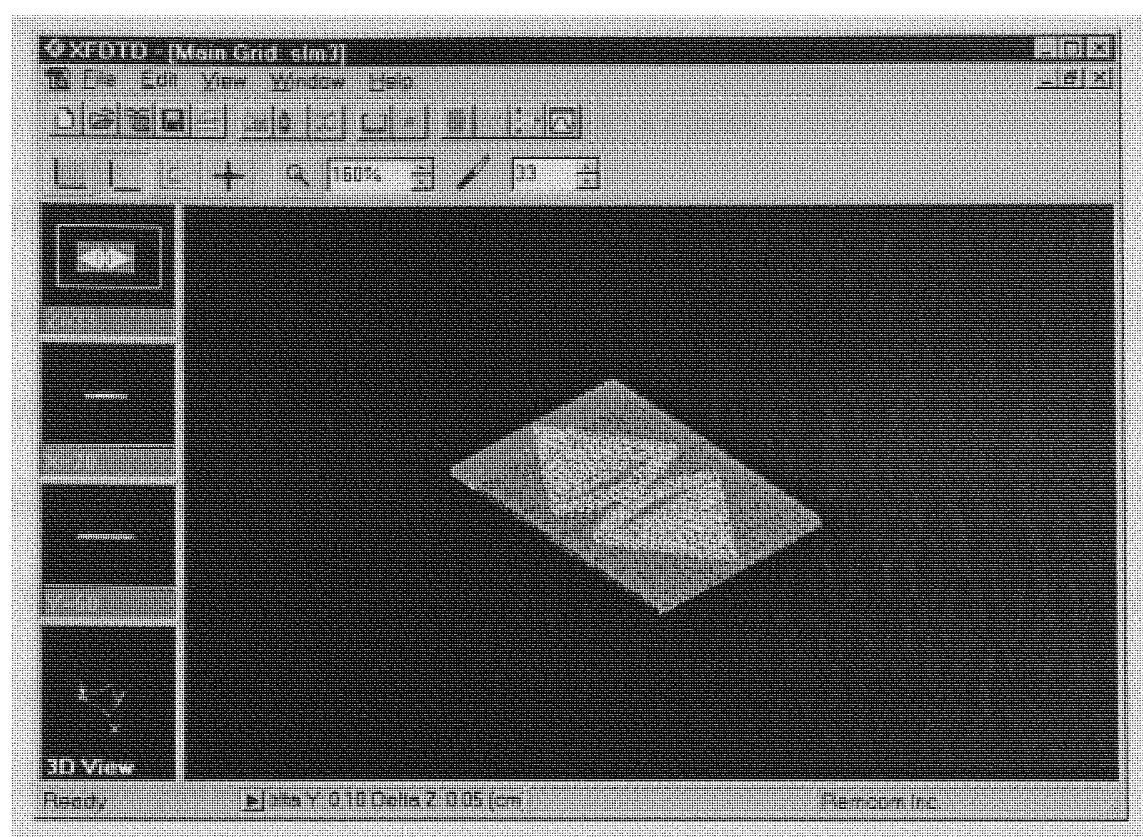

Fig. 66. 3-Dimensional view of SLMDIDO-C in XFDTD software.

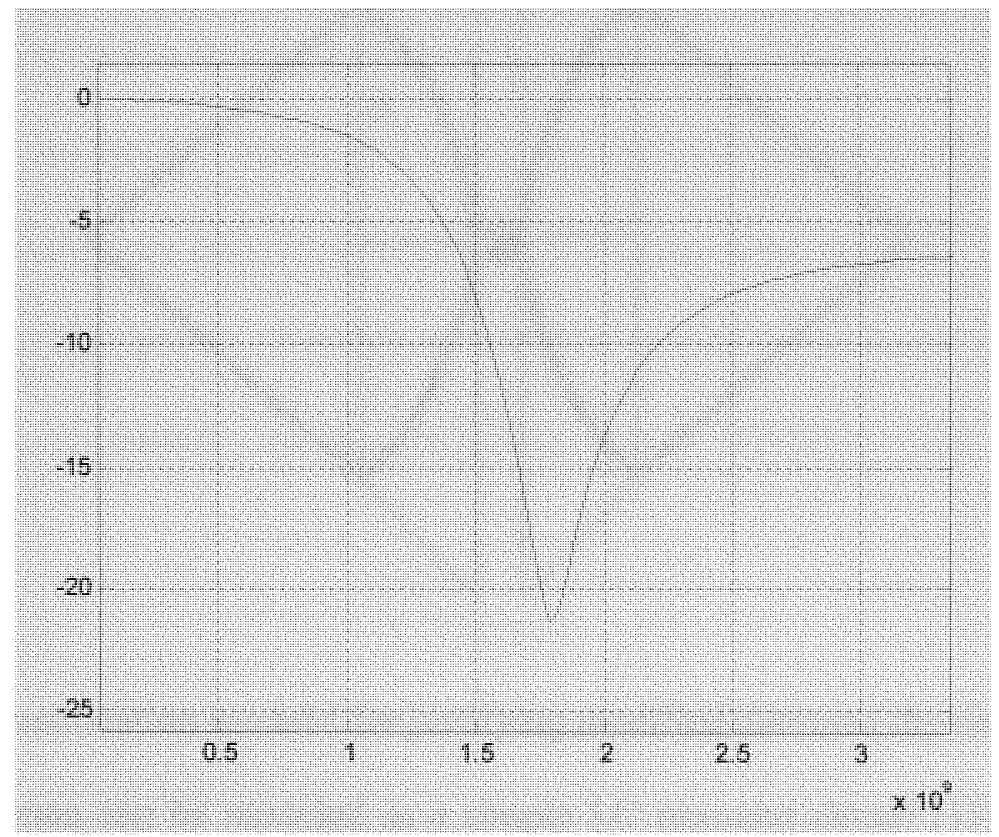

Fig. 67. Frequency Vs. return loss for SLMDIDO-C.

Fig. 63, Fig. 65, Fig. 67 show the return loss of SLMDIDO-A, SLMDIDO-B and SLMDIDO-C antenna models respectively. 


\section{CHAPTER 7}

\section{JUSTIFICATION}

\subsection{Justification for modified diamond dipole}

In order to justify the shape modification of diamond dipole, a blend of diamond dipole and modified diamond dipole as shown in Fig. 68 is considered. This shape modification is justified by taking the current density into account. Consider the portions $\mathrm{A}$ and $\mathrm{B}$. In diamond dipole the current density exists on both $\mathrm{A}$ and $\mathrm{B}$ regions. In case of modified diamond dipole, region A is absent (removed). Portion B has to accommodate the entire current present.

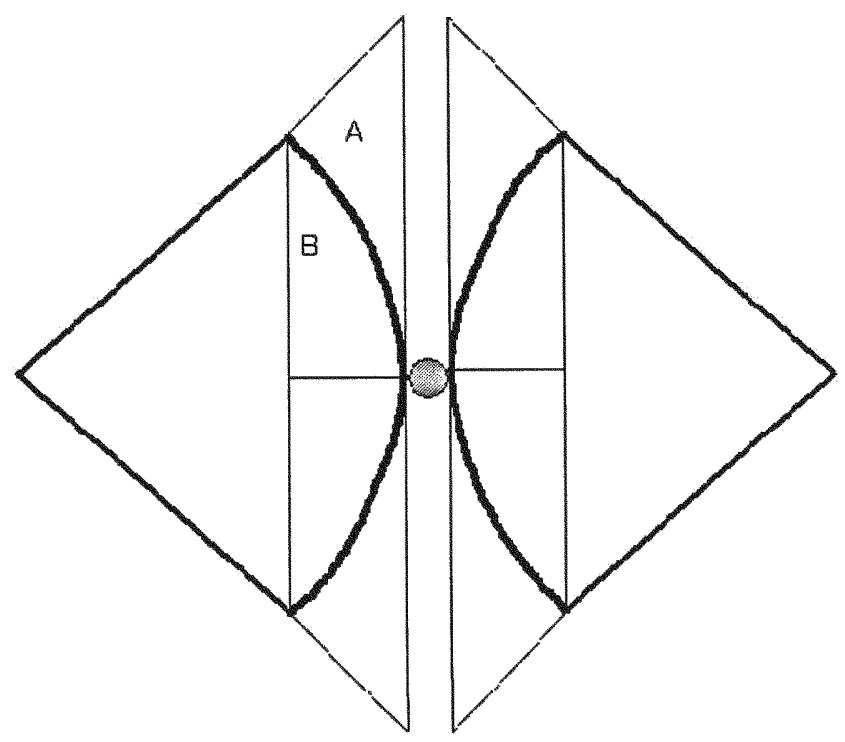

Fig. 68. Justification model-I for justifying modified diamond dipole behavior.

Any patch antenna can be modeled as a simple LC resonant circuit [15]. There will be different current densities at different portions of the antenna structure and these $\mathrm{L}$ and $\mathrm{C}$ values of the resonant circuit depends on the strength of these current densities, and the path and distance they travel on the antenna structure. The introduction of a circular arc 
base has a varied inductive effect. The modified diamond dipole can be divided into 5 regions as shown in Fig. 69 In region R1, diamond dipole and modified diamond dipole have almost the same size, and hence the current path is same for both the antennas in R1. In regions R2 and R4, modified diamond dipole has smaller area than diamond dipole and hence the current path is reduced (current density is increased). This can be modeled as a reduction in the inductance (shown in Fig. 70). In regions R3 and R4 modified diamond dipole has a much smaller area than diamond dipole which accounts for much smaller current paths and a still reduced inductance. The equivalent circuits are shown in Fig. 70. Hence the antenna acts a triple resonant circuit. This is the case for smaller flare angles. But if either the flare angle or the radius of curvature increases to a larger extent, it has the effect of increasing the arc length. In such case the antenna can be modeled as a resonant circuit of more than three resonant frequencies, since with the increase of the flare angle, the arc length increases (Fig. 69) and hence the antenna can be divided into more number of regions and hence modeled as an equivalent resonant circuit consisting of more resonant frequencies. Two antenna models, the diamond dipole antenna as shown in section 6.2 of chapter VI and MDIDO-Al are considered for explaining the justification

\section{Table 3}

Information about the antenna models selected for justification I

\begin{tabular}{|c|c|c|}
\hline Antenna Model & Semi-flare angle $(\propto / 2)^{0}$ & Radius of curvature (R mm) \\
\hline DIDO & 27 & 0 \\
\hline MDIDO-A1 & 27 & 20 \\
\hline
\end{tabular}




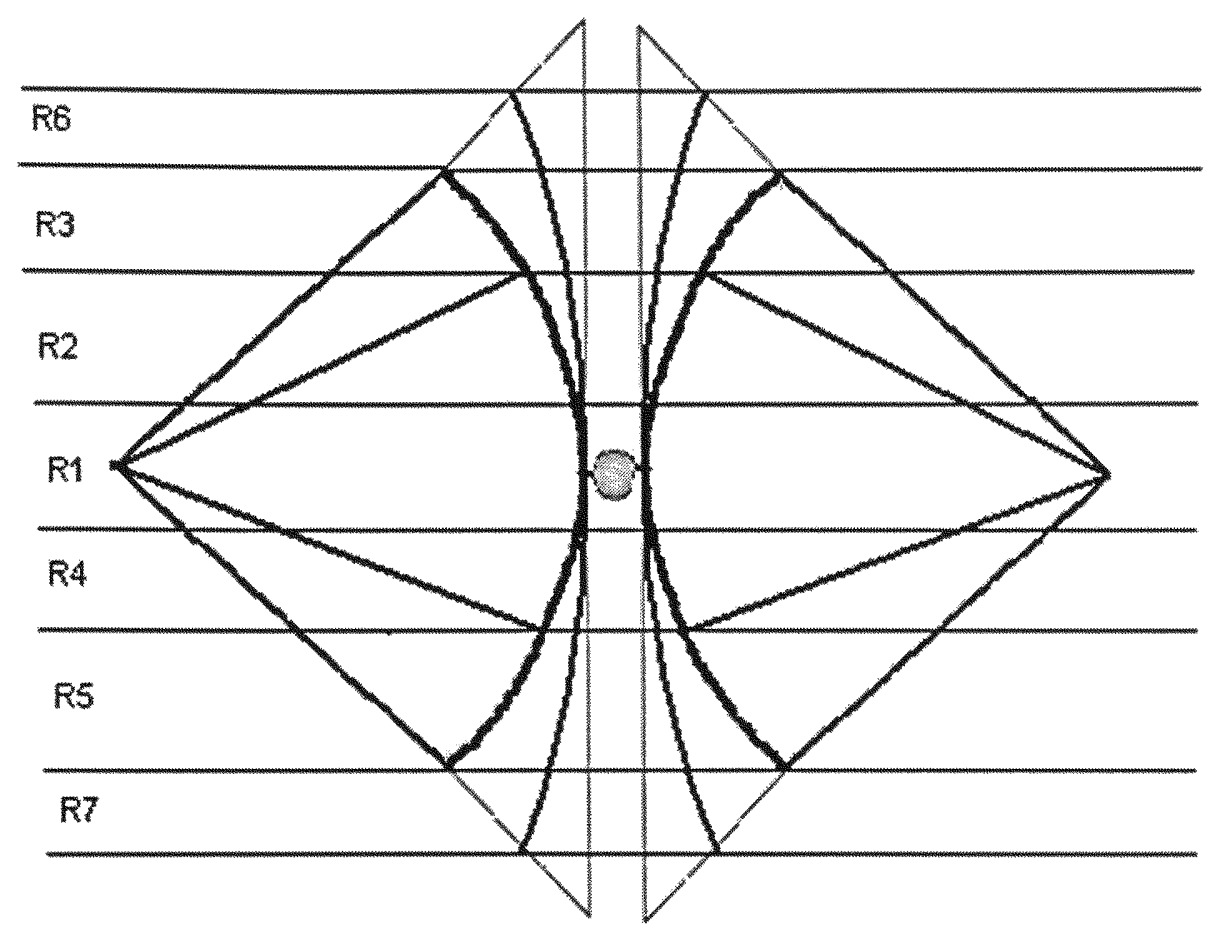

Fig. 69. Justification model-II for justifying modified diamond dipole behavior.

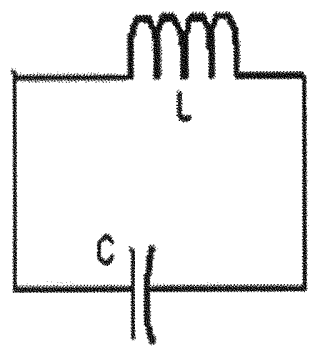

Equivalent circuit of region R1

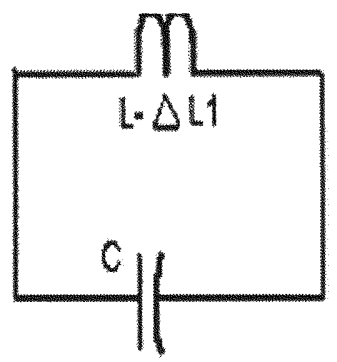

Equivalent circuit of regions R2, R4

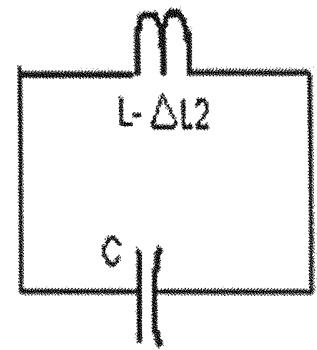

Equivalent circuit of regions $R 3, R 5$

Fig.70. Resonant circuits for justification model-II.

Fig. 72 shows a comparison of current densities measured at a point $(60,60,33)$ of DIDO (diamond dipole) and MDIDO-A1. It can be observed that the current density is higher for modified diamond dipole than for modified diamond dipole. 


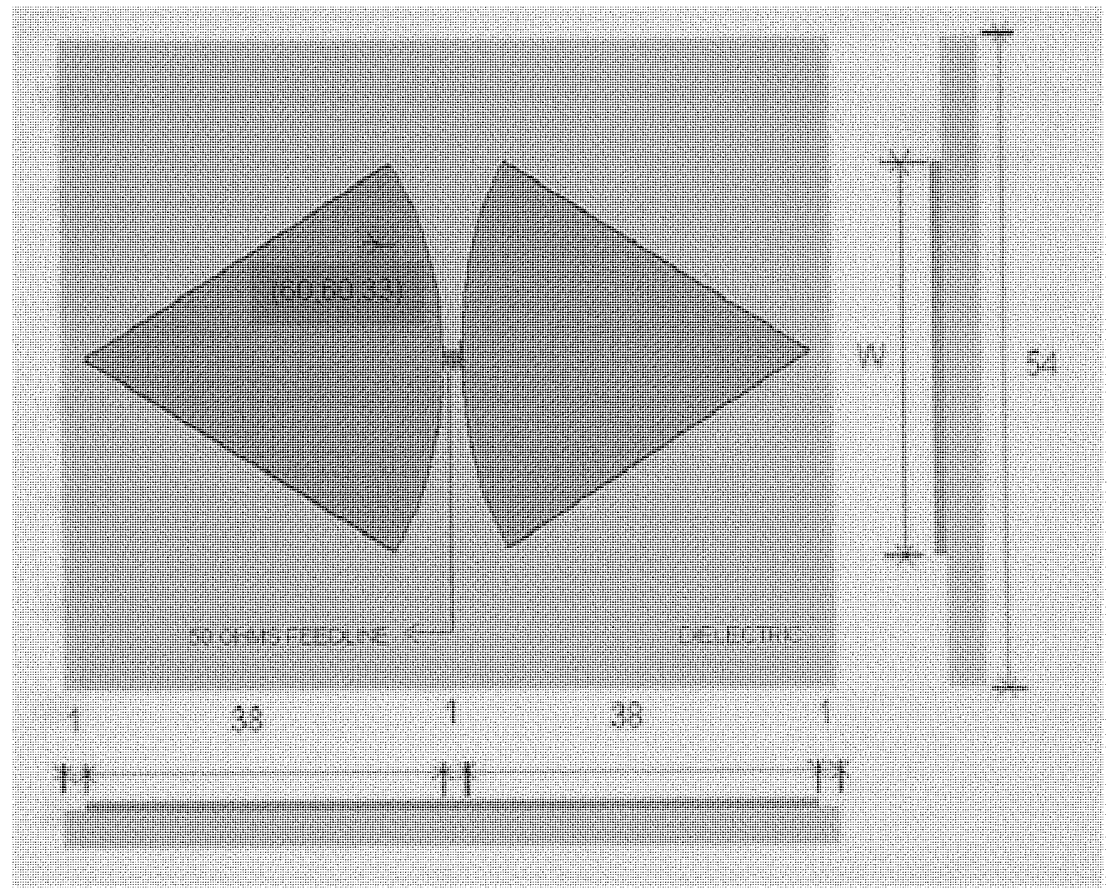

Fig. 71. Coordinate where current density (Jx is measured).

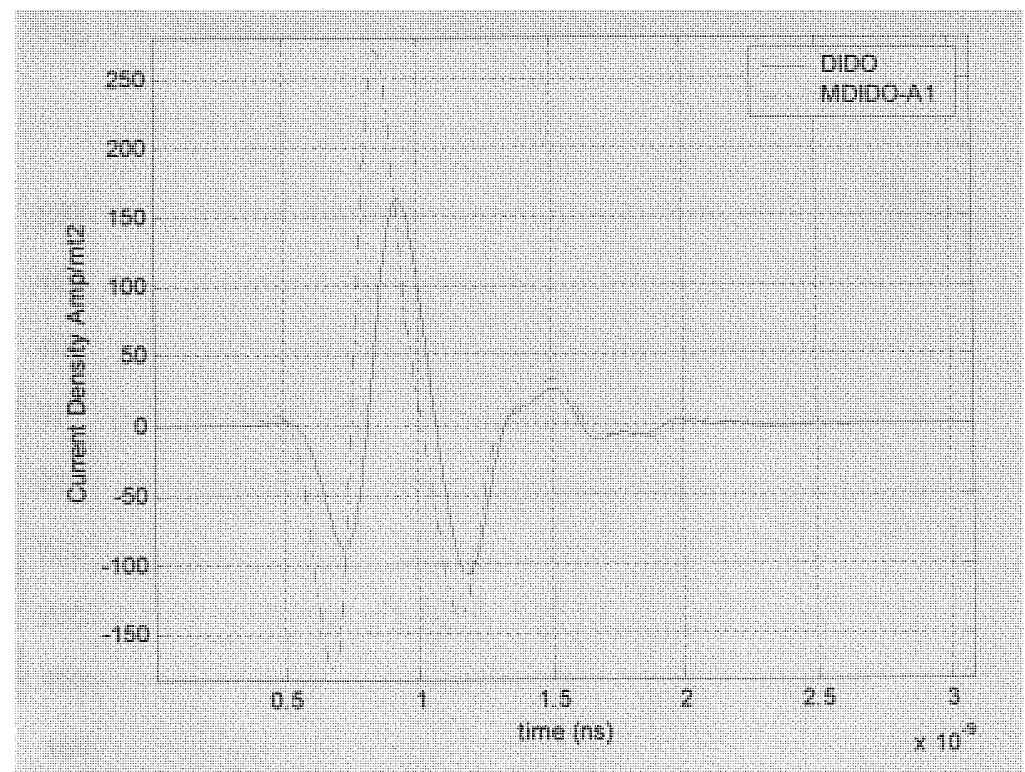

Fig. 72. Current density for DIDO and MDIDO-A1.

Fig. 73, Fig. 74 and Fig. 75 show comparison of return loss, input resistance and input reactance of DIDO and MDIDO-A1 respectively. 


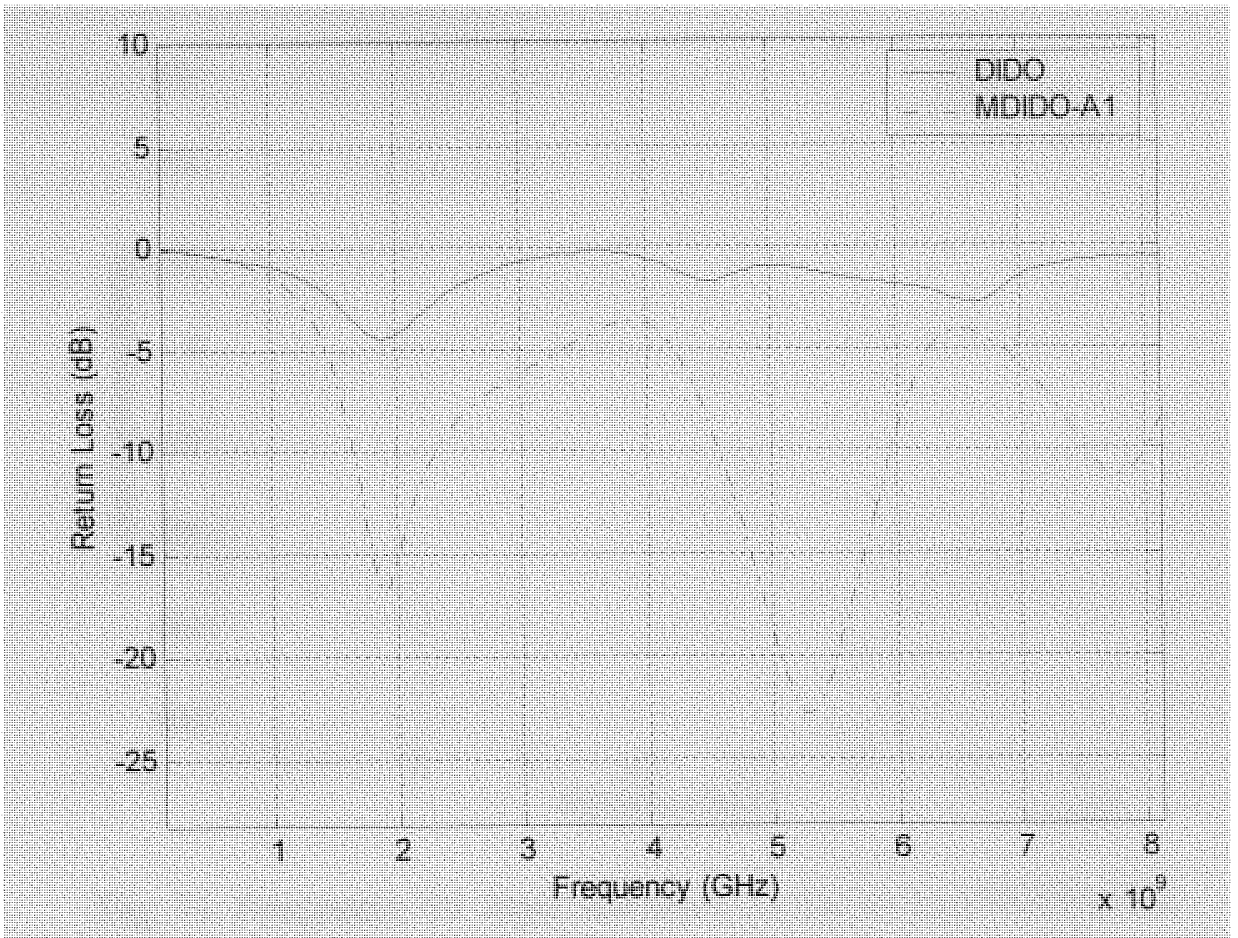

Fig. 73. Frequency Vs. return loss for DIDO and MDIDO-A1.

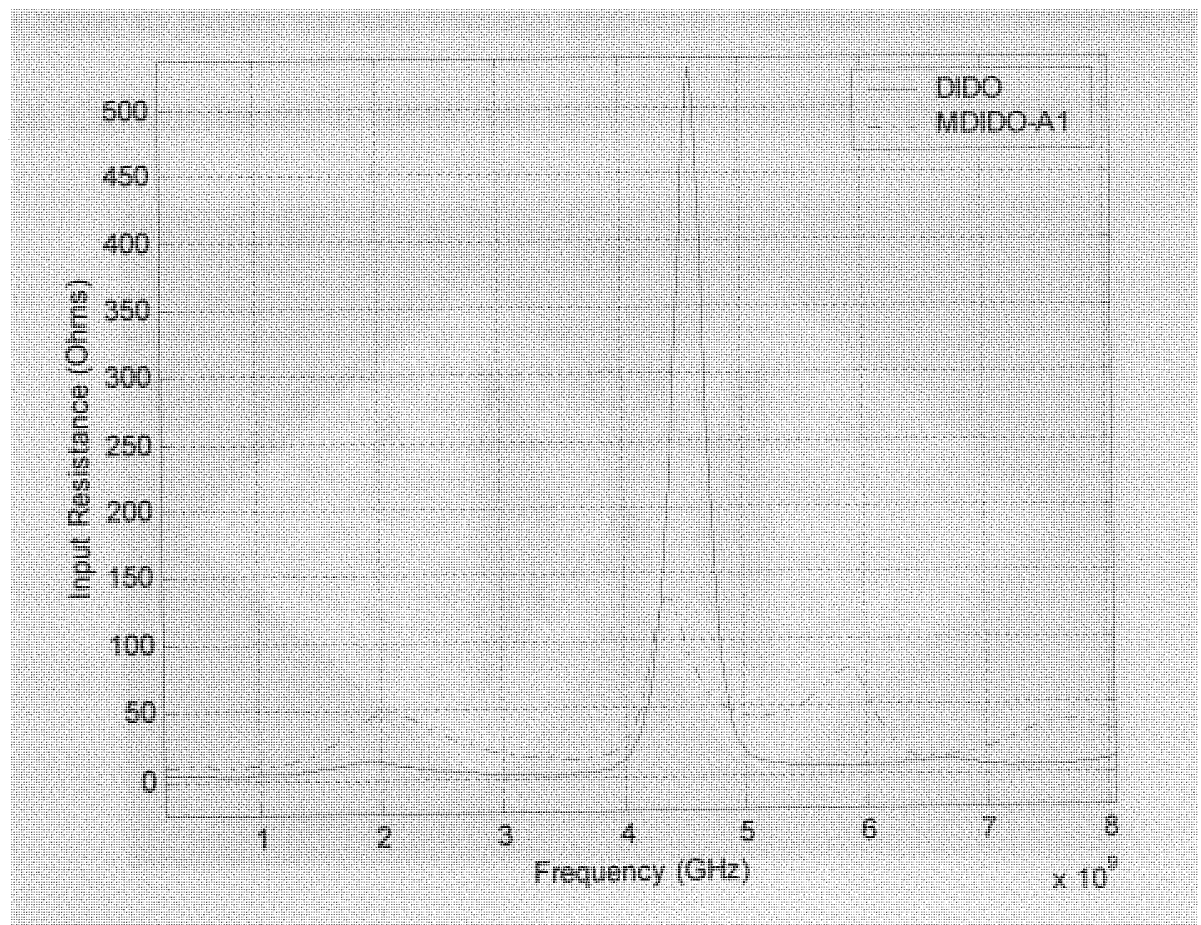

Fig. 74. Frequency Vs. input resistance for DIDO and MDIDO-A1. 


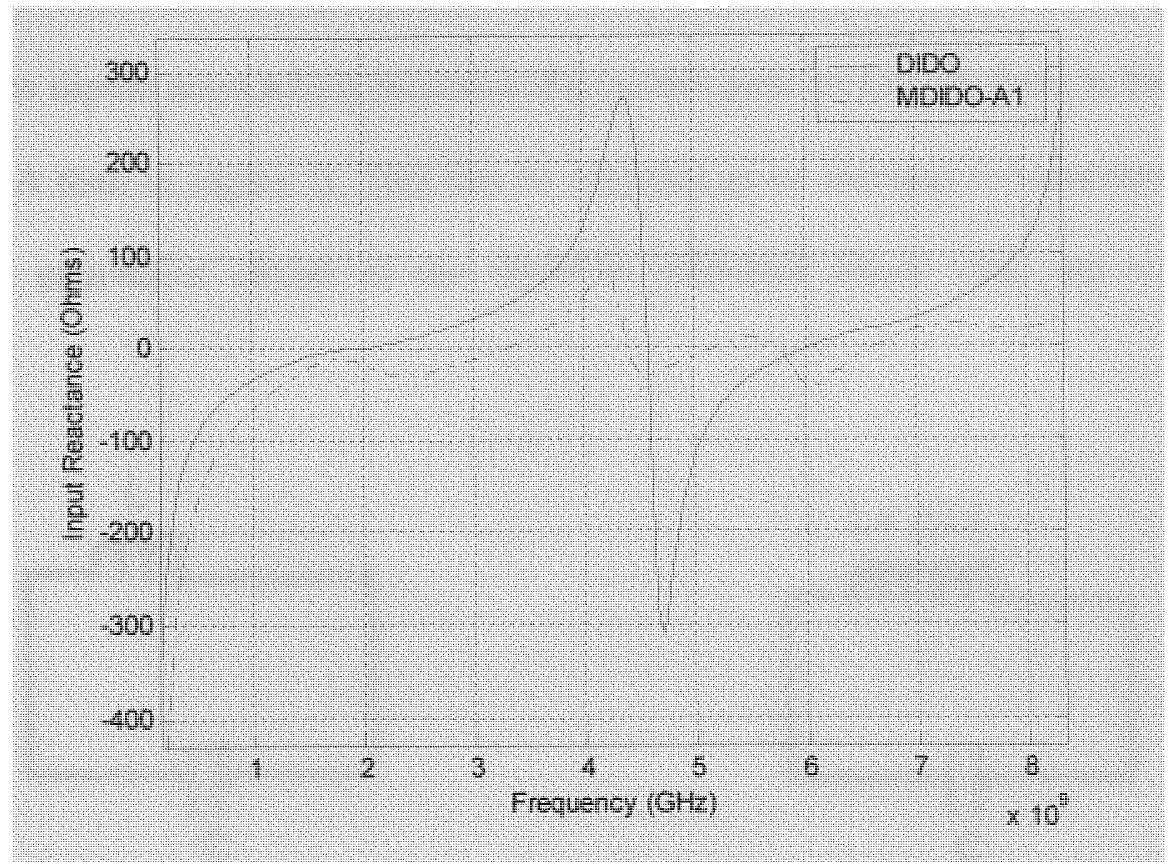

Fig. 75. Frequency Vs. input reactance for DIDO and MDIDO-A1.

\subsection{Justification for slot loaded modified diamond dipole}

In order to justify the slot loading applied to modified diamond dipole, Fig. 76 is considered. Current density is taken into account for justification purpose.

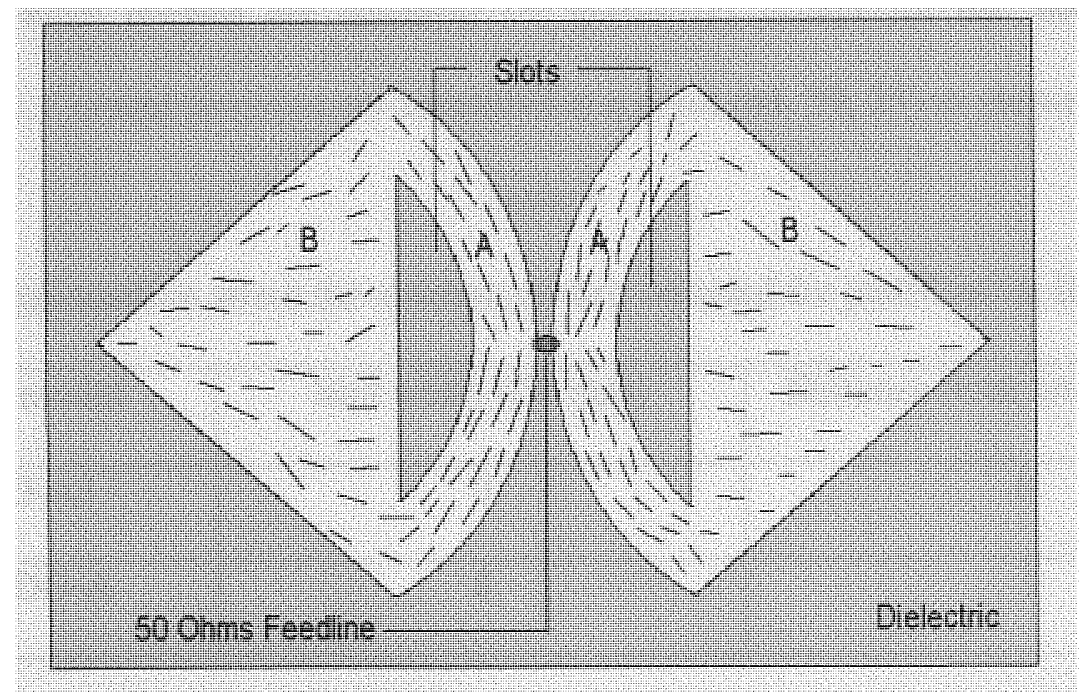

Fig. 76. Current distribution in slot loaded modified diamond dipole. 
When slots are introduced, the current must traverse round the slot to travel the through the dipole arms which increases the current path. In Fig. 76 there is more current in region $\mathrm{A}$ than in region $\mathrm{B}$. This can be modeled as an increase in inductance. Fig. 77 shows an equivalent circuit of the modified diamond dipole with and without slot loading.

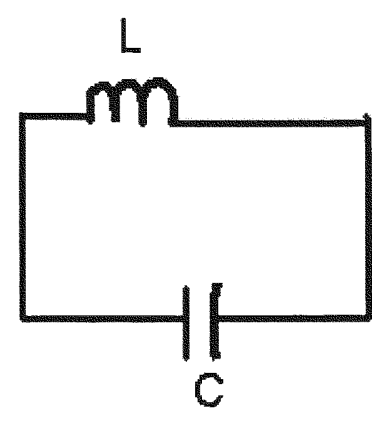

Fig. 77. Equivalent circuit of modified diamond dipole.

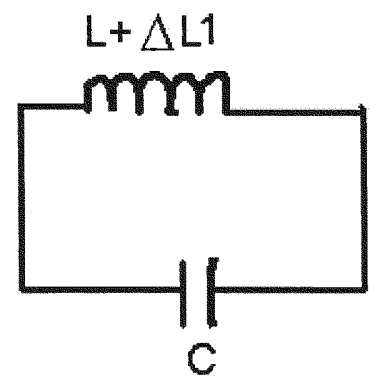

Fig. 78. Equivalent circuit of slot loaded modified diamond dipole.

Two antenna models MDIDO-A1 and SLMDIDO-A are considered to explain the justification. Both models are identical (in geometry) except for the slot present in SLMDIDO-A. Current densities are measured at a point $(64,39,33)$ as shown in Fig. 79.

Table 4

Information about the antenna models selected for justification II

\begin{tabular}{|c|c|c|}
\hline Antenna model & Slot width (D1 mm) & Distance from feed (D2 mm) \\
\hline MDIDO-A1 & 0 & 0 \\
\hline SLMDIDO-A & 8 & 1 \\
\hline
\end{tabular}




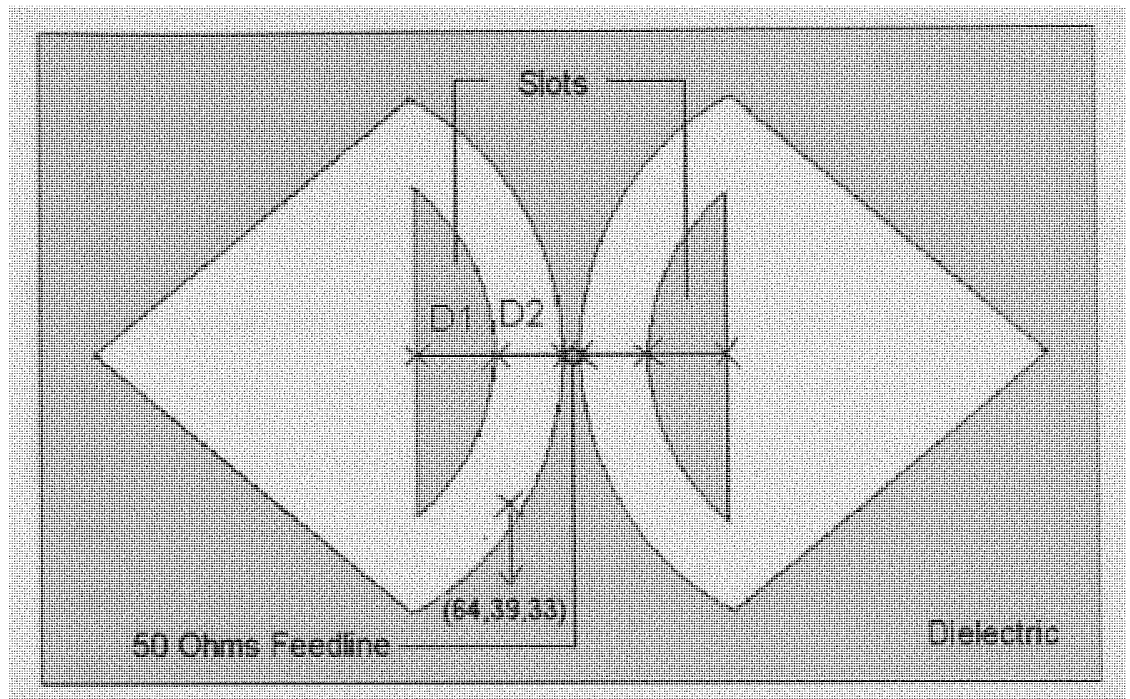

Fig. 79. Point where current density is measured.

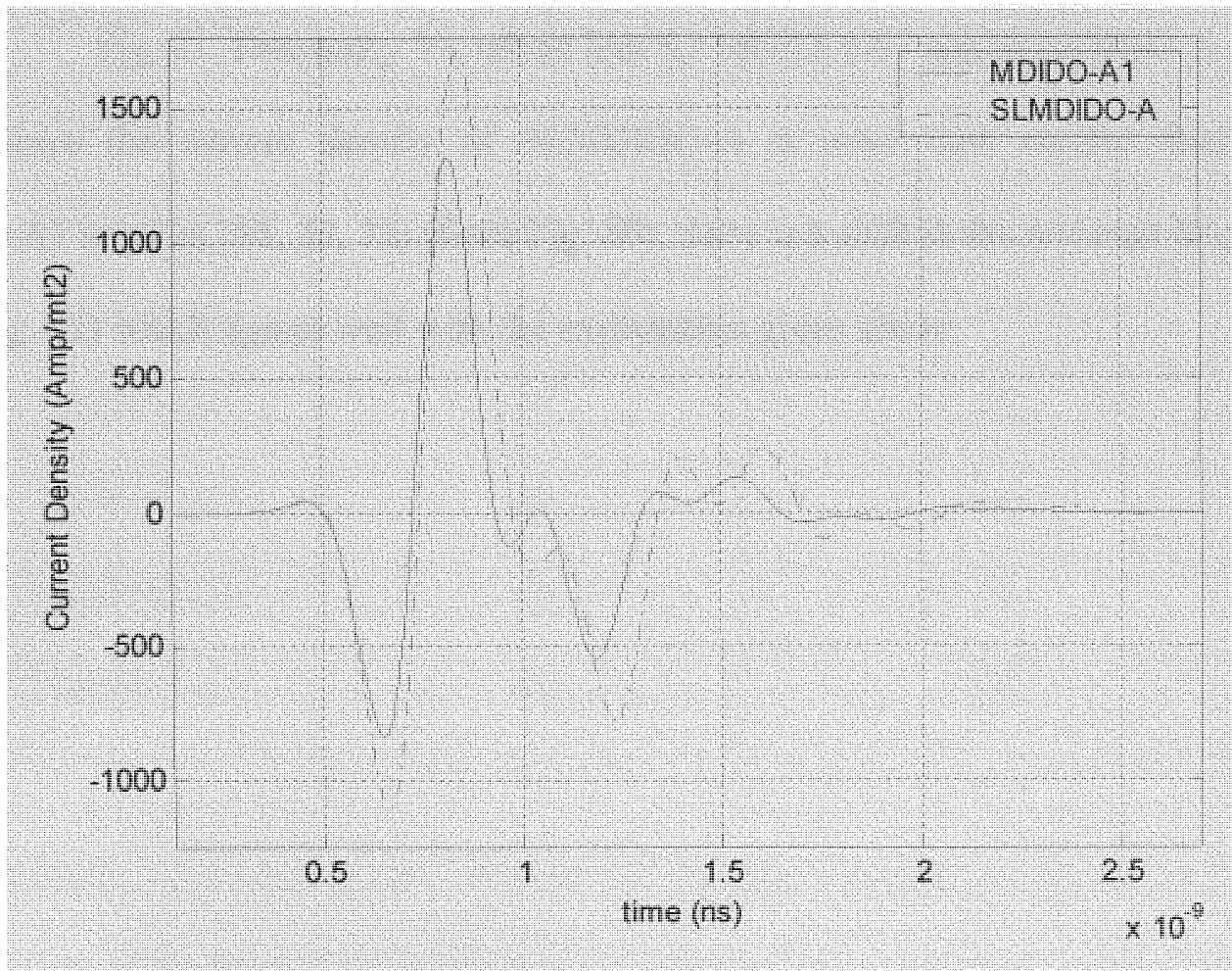

Fig. 80. Current densitiy for MDIDO-A1 and SLMDIDO-A. 


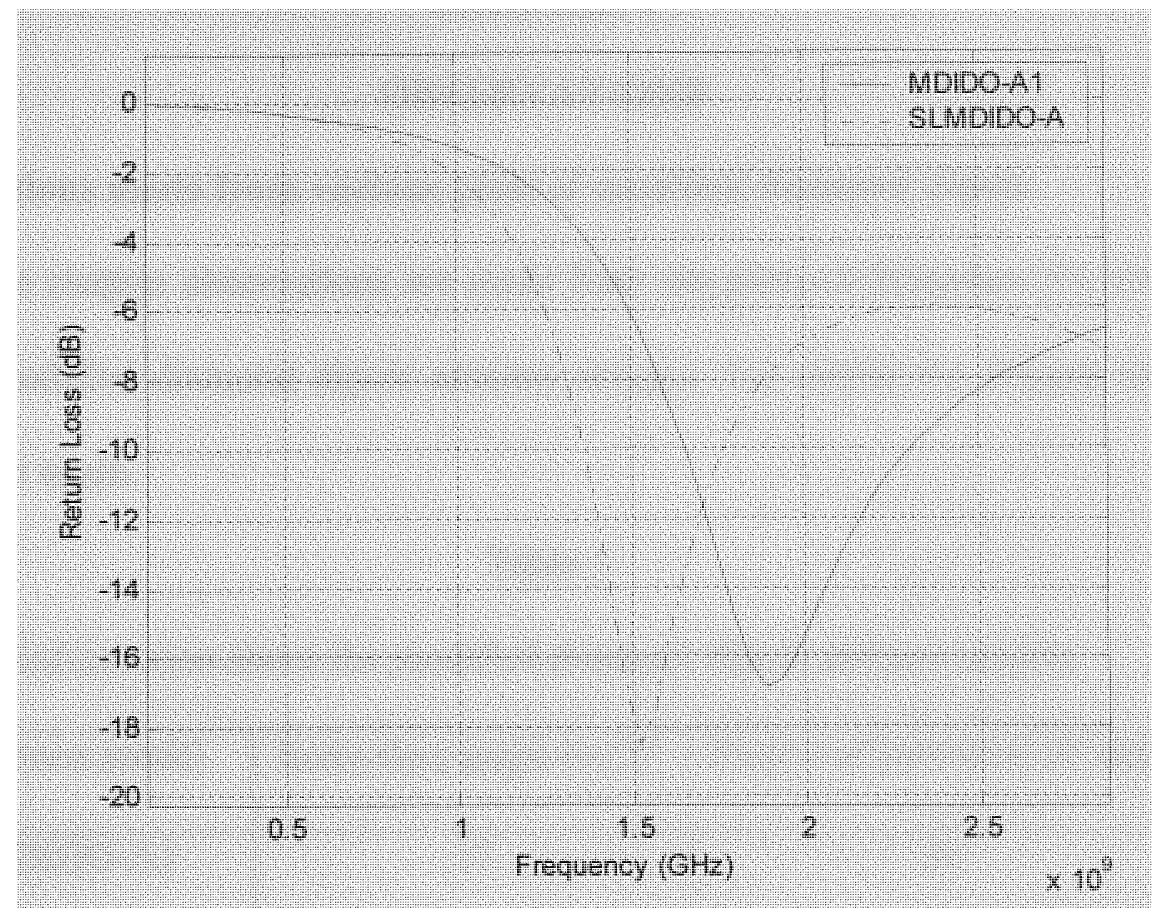

Fig. 81. Frequency Vs. return loss for MDIDO-A1 and SLMDIDO-A.

Since the current density of SLMDIDO-A is more than current density of MDIDO-A1 at point $(64,39,33)$ (from Fig. 80$)$, there is a shift in the resonant frequency (from Fig. 81) which agrees well with the explanation given above. 


\section{CHAPTER 8}

\section{OBSERVATIONS}

\subsection{Observations made from various plots of modified diamond dipole}

In this chapter observations are made from the plots of frequency Vs. return loss of various modified diamond dipole antenna designs. For clarity the designs names are provided here again.

\section{Table 5}

Information about the modified diamond dipole antenna models

\begin{tabular}{|c|c|c|}
\hline Antenna model & Semi-flare angle $(\propto / 2)^{0}$ & Radius of curvature $(\mathrm{R} \mathrm{mm})$ \\
\hline MDIDO-A1 & 27 & 20 \\
\hline MDIDO-A2 & 27 & 30 \\
\hline MDIDO-A3 & 27 & 40 \\
\hline MDIDO-B1 & 35 & 20 \\
\hline MDIDO-B2 & 35 & 30 \\
\hline MDIDO-B3 & 35 & 40 \\
\hline MDIDO-C1 & 45 & 20 \\
\hline MDIDO-C2 & 45 & 30 \\
\hline MDIDO-C3 & 45 & 40 \\
\hline
\end{tabular}

Initially, flare angle is kept constant and antennas are analyzed for different radius of curvatures of the base of triangular arms of modified diamond dipole. Hence MDIDOA1, MDIDO-A2, MDIDO-A3 (Fig. 82) and MDIDO-B1, MDIDO-B2, MDIDO-B3 (Fig. 85) and MDIDO-C1, MDIDO-C2, MDIDO-C3 (Fig. 88) are compared individually for 
return loss (S11). Next, radius of curvature of the base of triangular arms of modified diamond dipole is kept constant and the flare angle is modified. Hence MDIDO-A1, MDIDO-B1, MDIDO-C1 (Fig. 91) and MDIDO-A2, MDIDO-B2, MDIDO-C2 (Fig. 94) and MDIDO-A3, MDIDO-B3, MDIDO-C3 (Fig. 97) are compared for return loss (S11).

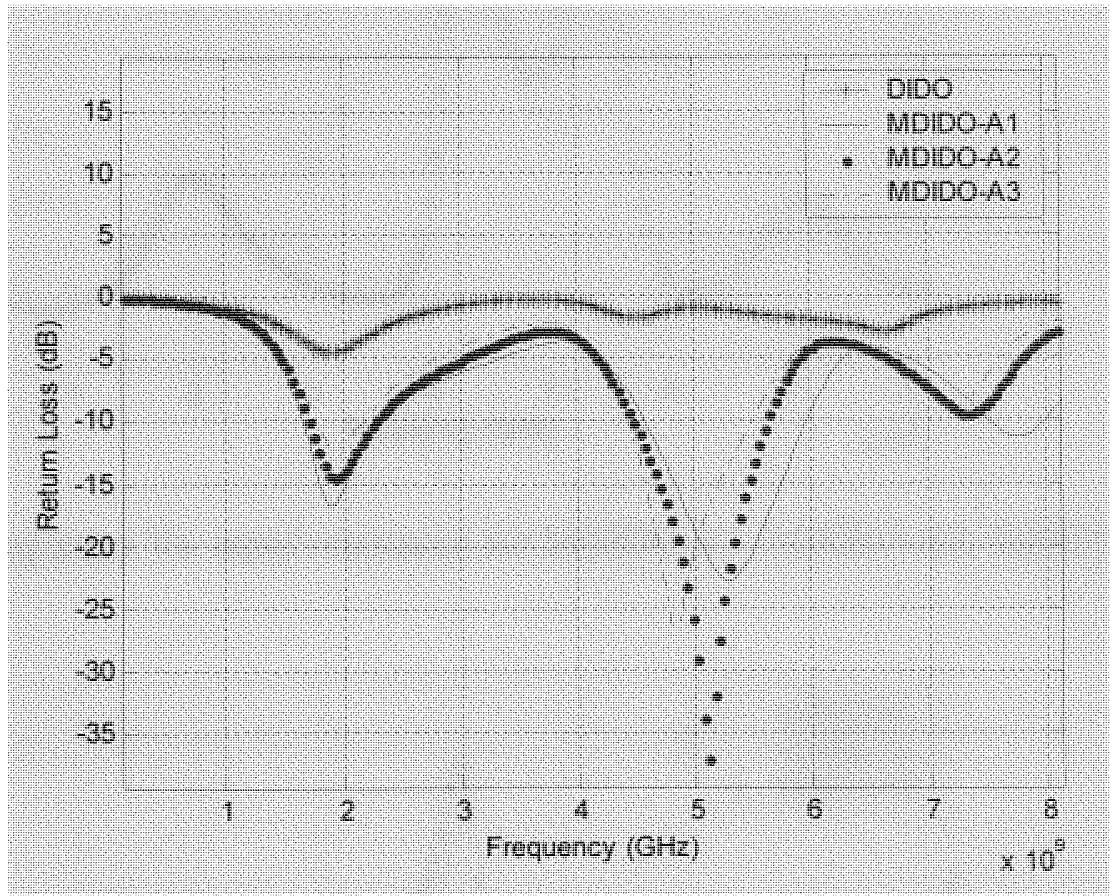

Fig. 82. Frequency Vs. return loss for MDIDO-A1, MDIDO-A2, MDIDO-A3.

Table 6

Resonant frequencies of MDIDO-A series antennas

\begin{tabular}{|c|c|}
\hline Antenna model & Frequencies of resonance $(\mathrm{GHz})$ \\
\hline DIDO & 1.9 \\
\hline MDIDO-A1 & $1.9,5.3,7.7$ \\
\hline MDIDO-A2 & $1.9,5.1,7.3$ \\
\hline MDIDO-A3 & $1.9,4.8,7.2$ \\
\hline
\end{tabular}




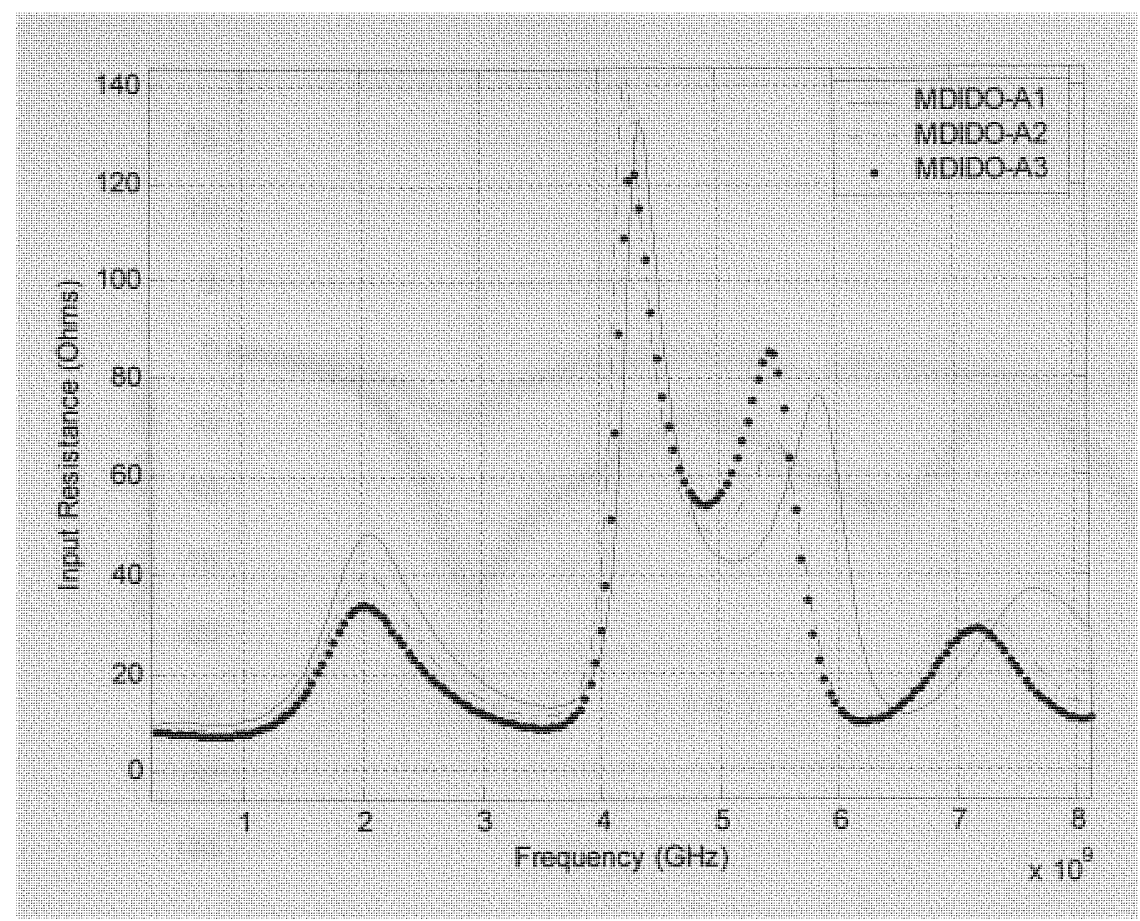

Fig. 83. Frequency Vs. input resistance for MDIDO-A1, MDIDO-A2, MDIDO-A3.

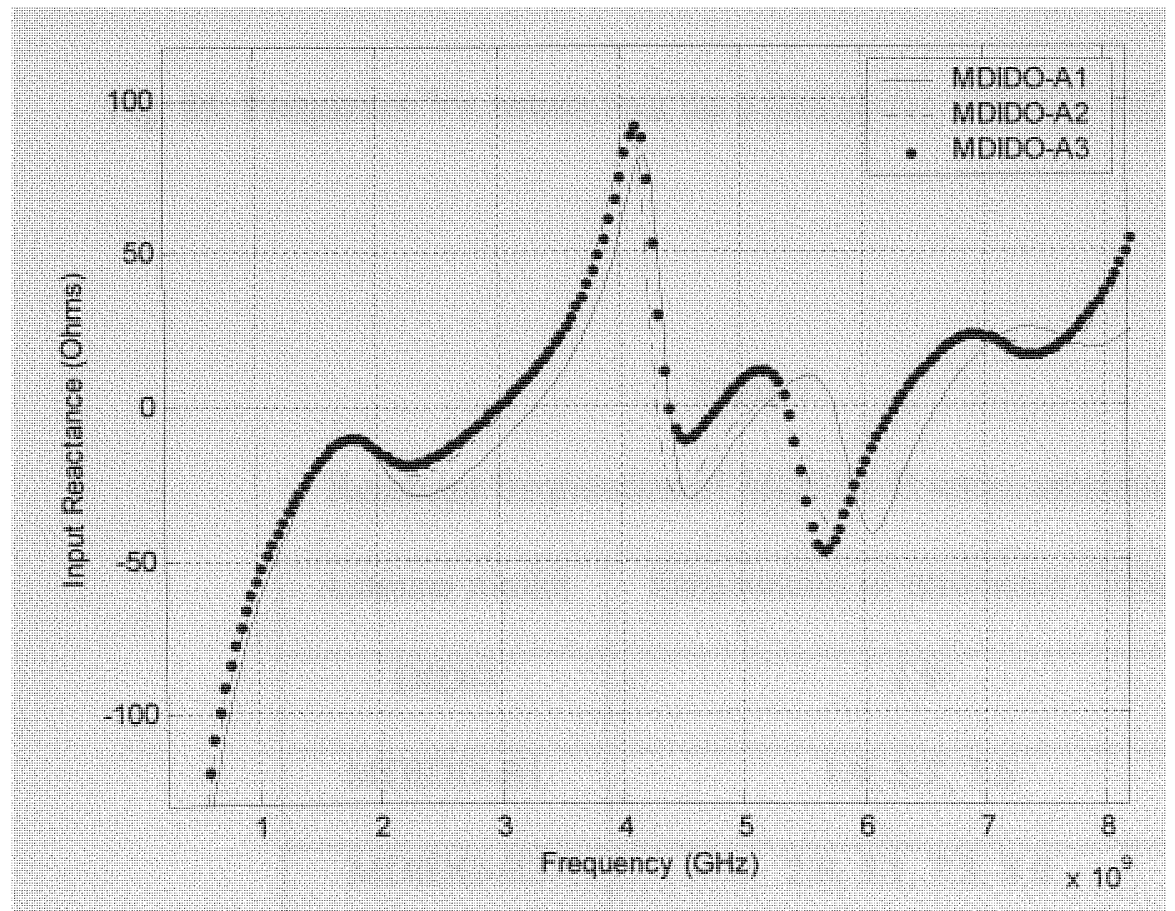

Fig. 84. Frequency Vs. input reactance for MDIDO-A1, MDIDO-A2, MDIDO-A3. 


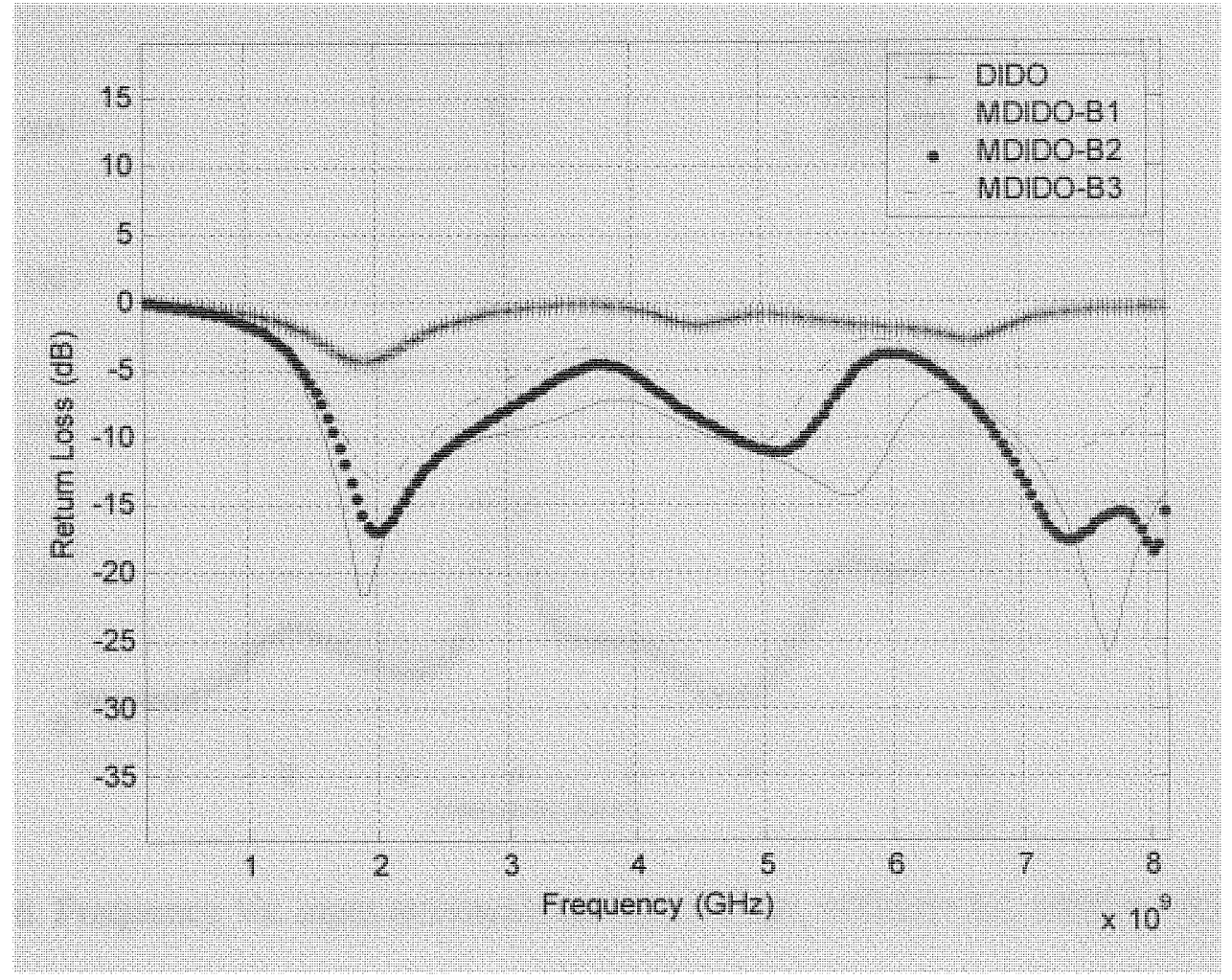

Fig. 85. Frequency Vs. return loss for MDIDO-B1, MDIDO-B2, MDIDO-B3.

Table 7

Resonant frequencies of MDIDO-B series antennas

\begin{tabular}{|c|c|}
\hline Antenna model & Frequencies of resonance (GHz) \\
\hline DIDO & 1.9 \\
\hline MDIDO-B1 & $1.9,5.7,7.6$ \\
\hline MDIDO-B2 & $2.0,5.1,7.3,8.0$ \\
\hline MDIDO-B3 & $2.0,4.9,7.1$ \\
\hline
\end{tabular}




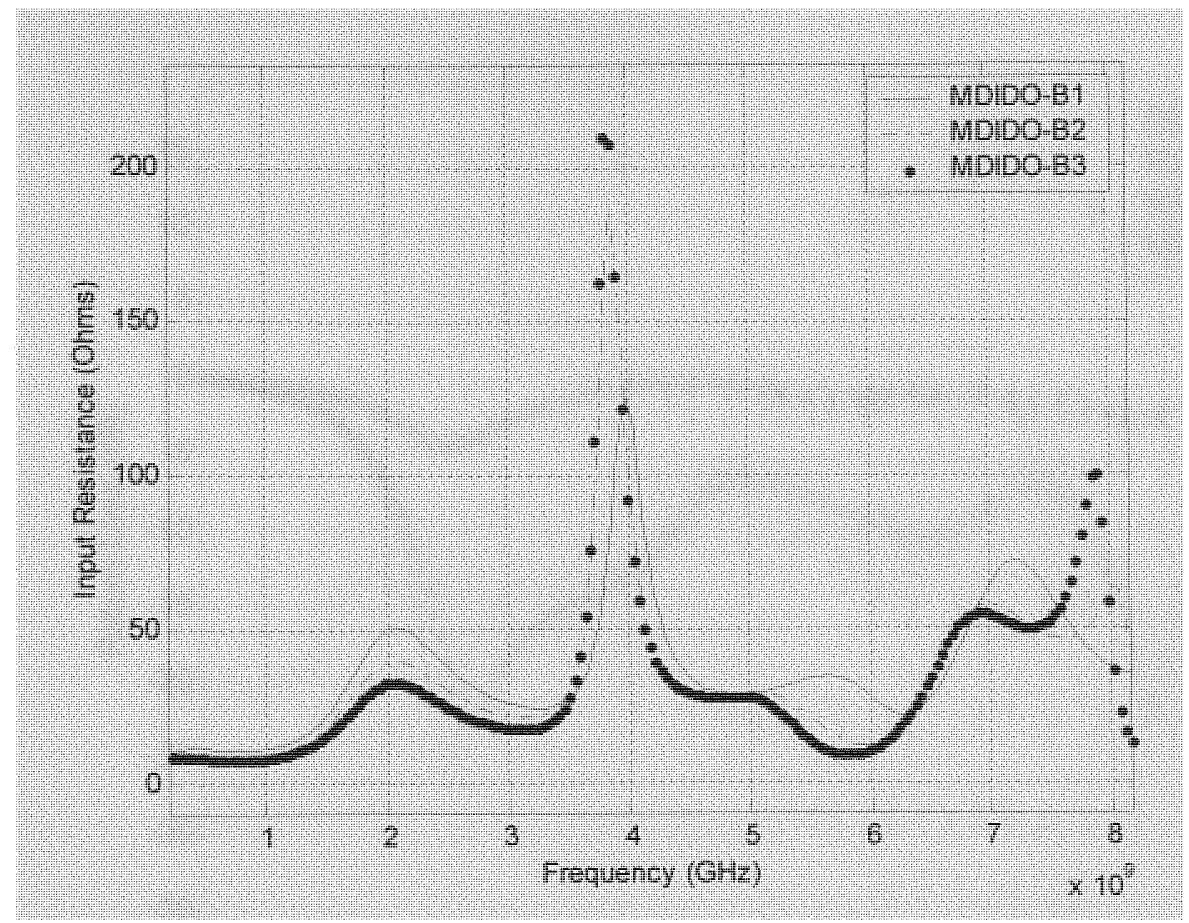

Fig. 86. Frequency Vs. input resistance for MDIDO-B1, MDIDO-B2, MDIDO-B3.

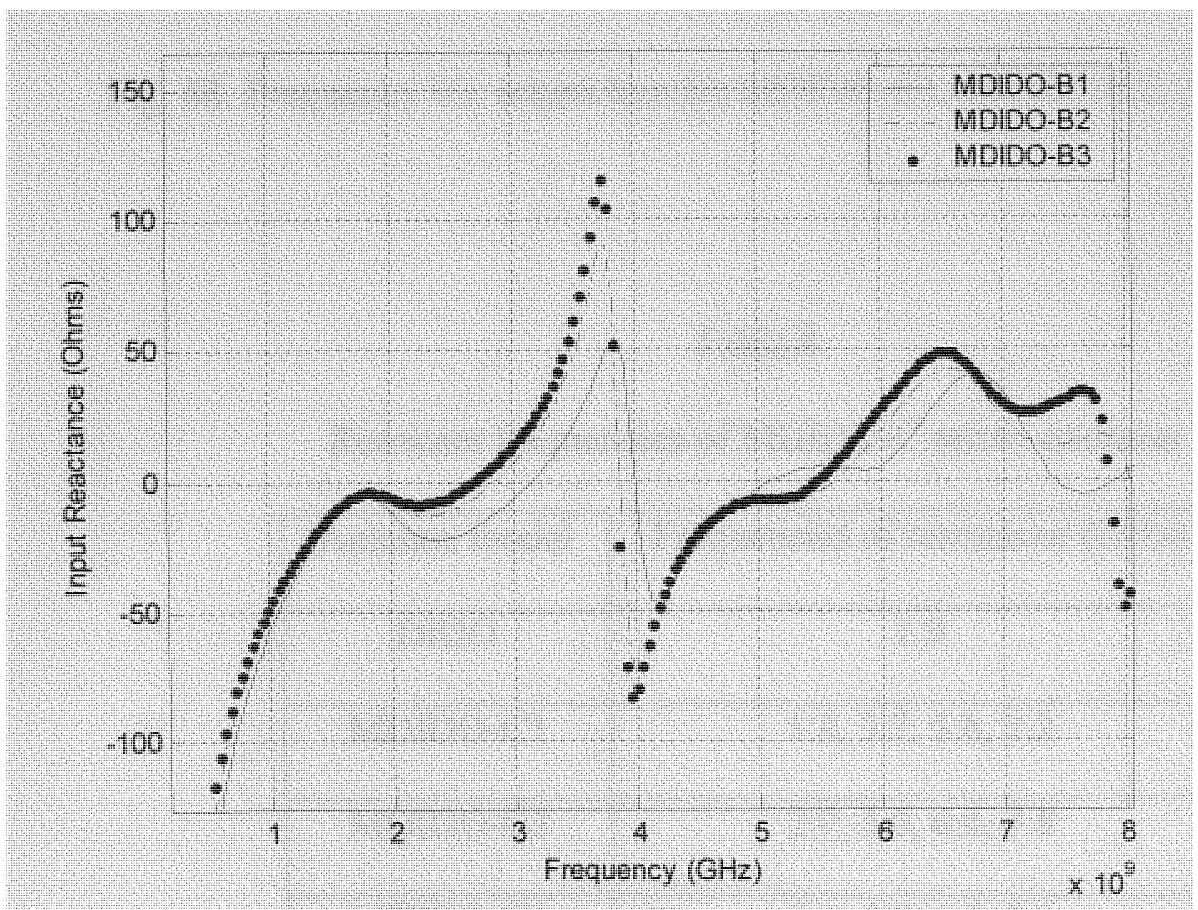

Fig. 87. Frequency Vs. input reactance for MDIDO-B1, MDIDO-B2, MDIDO-B3. 


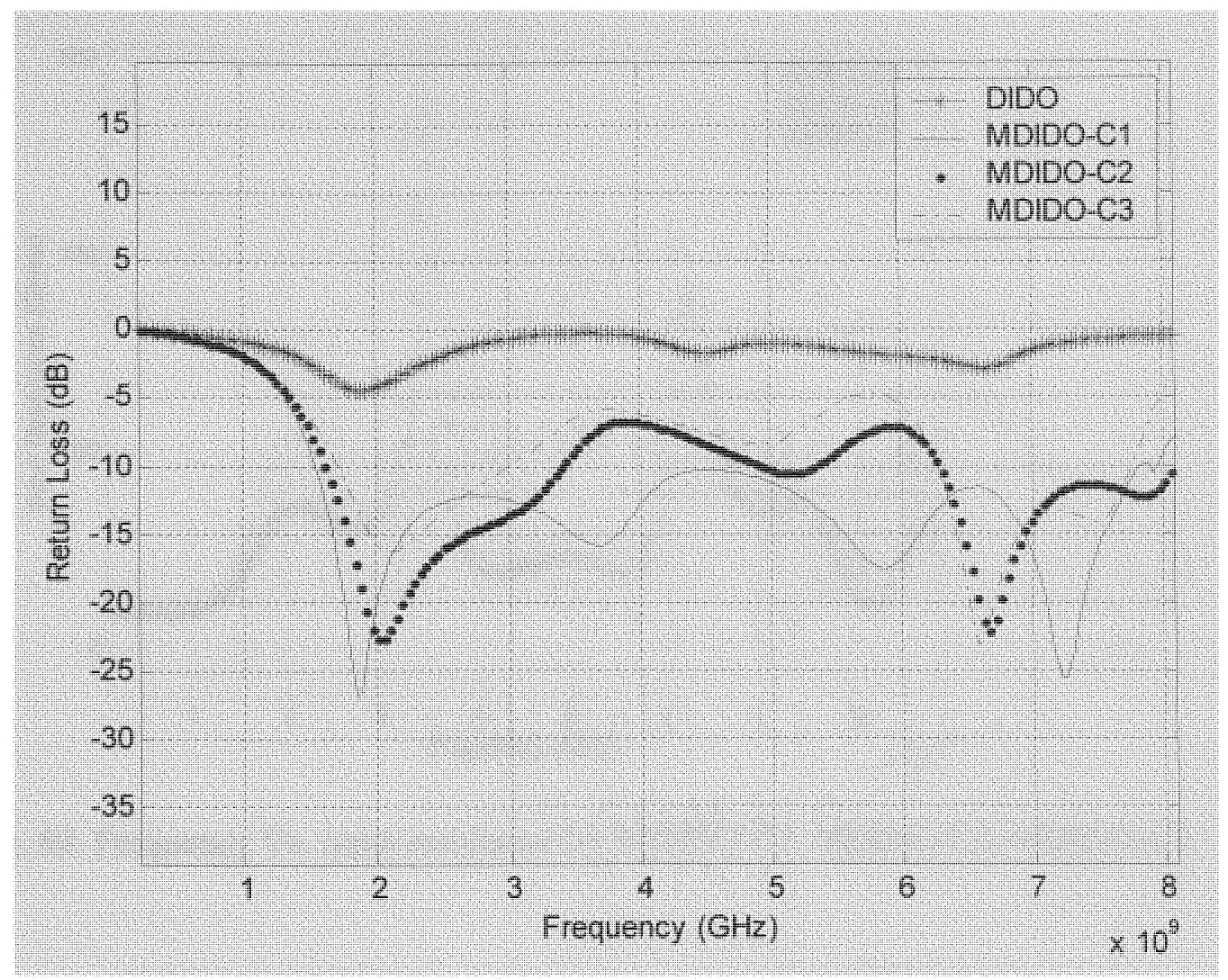

Fig. 88. Frequency Vs. return loss for MDIDO-C1, MDIDO-C2, MDIDO-C3.

Table 8

Resonant frequencies of MDIDO-C series antennas

\begin{tabular}{|c|c|}
\hline Antenna model & Frequencies of resonance (GHz) \\
\hline DIDO & 1.9 \\
\hline MDIDO-C1 & $1.8,3.7,5.8,7.2$ \\
\hline MDIDO-C2 & $2.0,5.0,6.6,7.8$ \\
\hline MDIDO-C3 & $2.1,5.0,6.6,7.5$ \\
\hline
\end{tabular}




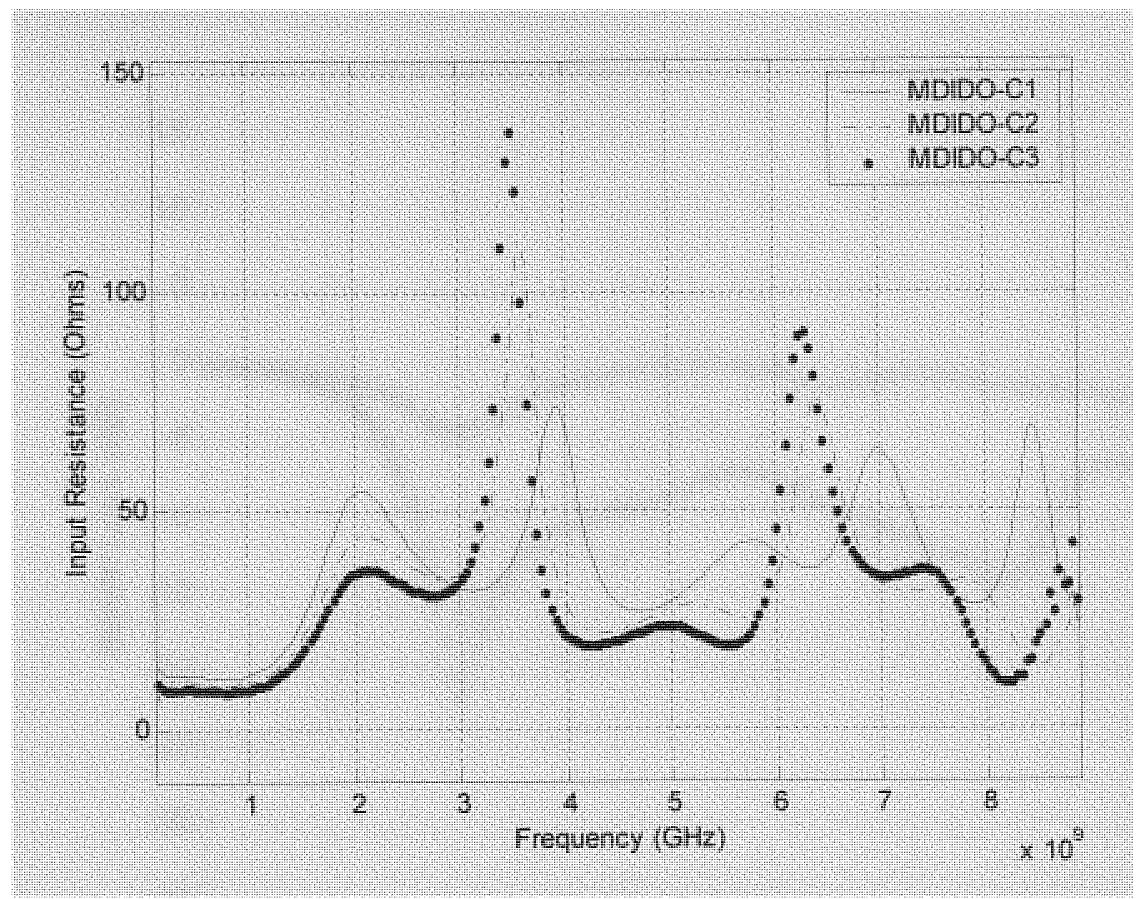

Fig. 89. Frequency Vs. input resistance for MDIDO-C1, MDIDO-C2, MDIDO-C3.



Fig. 90. Frequency Vs. input reactance for MDIDO-C1, MDIDO-C2, MDIDO-C3. 


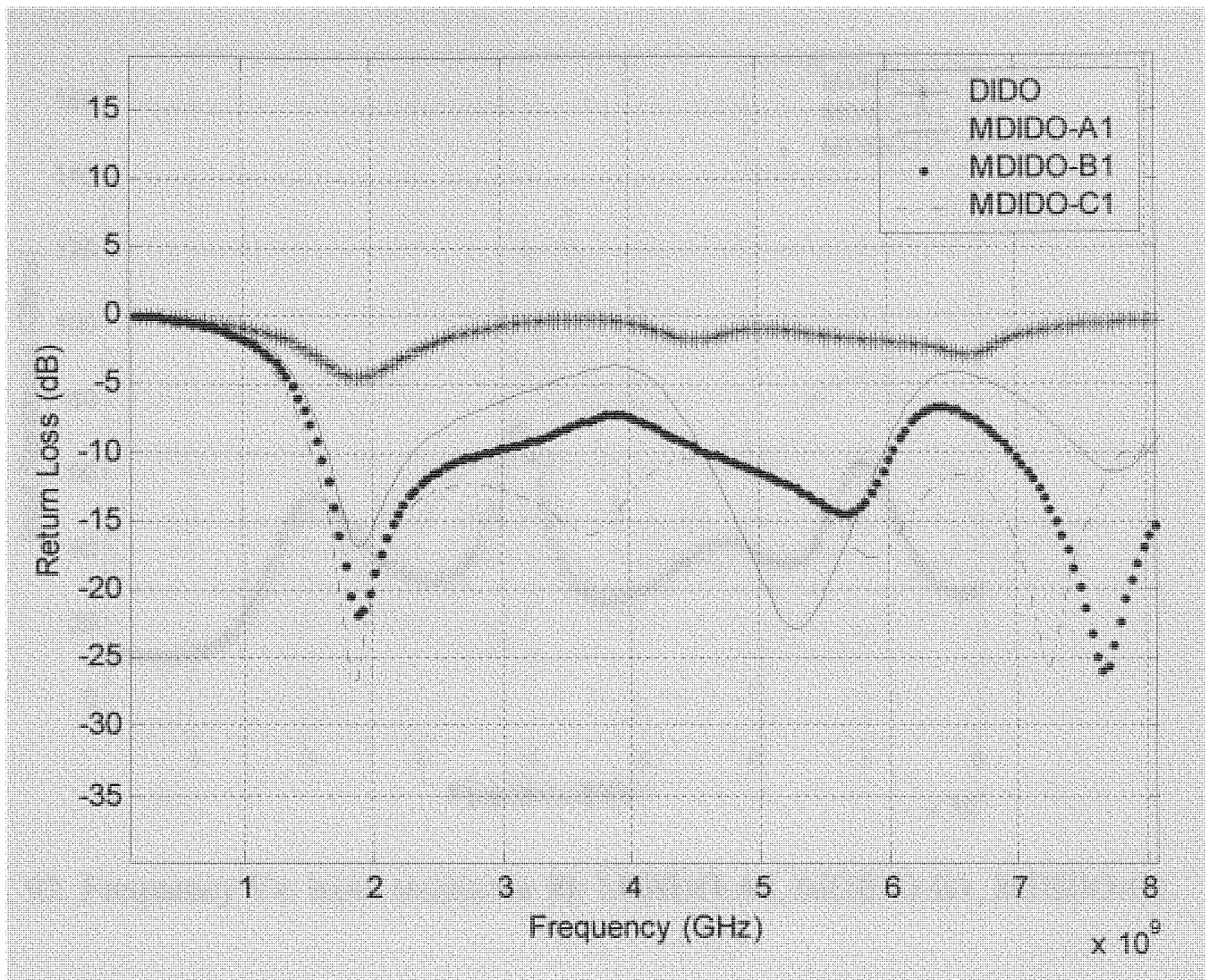

Fig. 91. Frequency Vs. return loss for MDIDO-A1, MDIDO-B1, MDIDO-C1.

Table 9

Resonant frequencies of MDIDO-A1, MDIDO-B1, MDIDOC1

\begin{tabular}{|c|c|}
\hline Antenna model & Frequencies of resonance $(\mathrm{GHz})$ \\
\hline DIDO & 1.9 \\
\hline MDIDO-A1 & $1.9,5.3,7.7$ \\
\hline MDIDO-B1 & $1.9,5.7,7.6$ \\
\hline MDIDO-C1 & $1.9,3.7,5.9,7.2$ \\
\hline
\end{tabular}




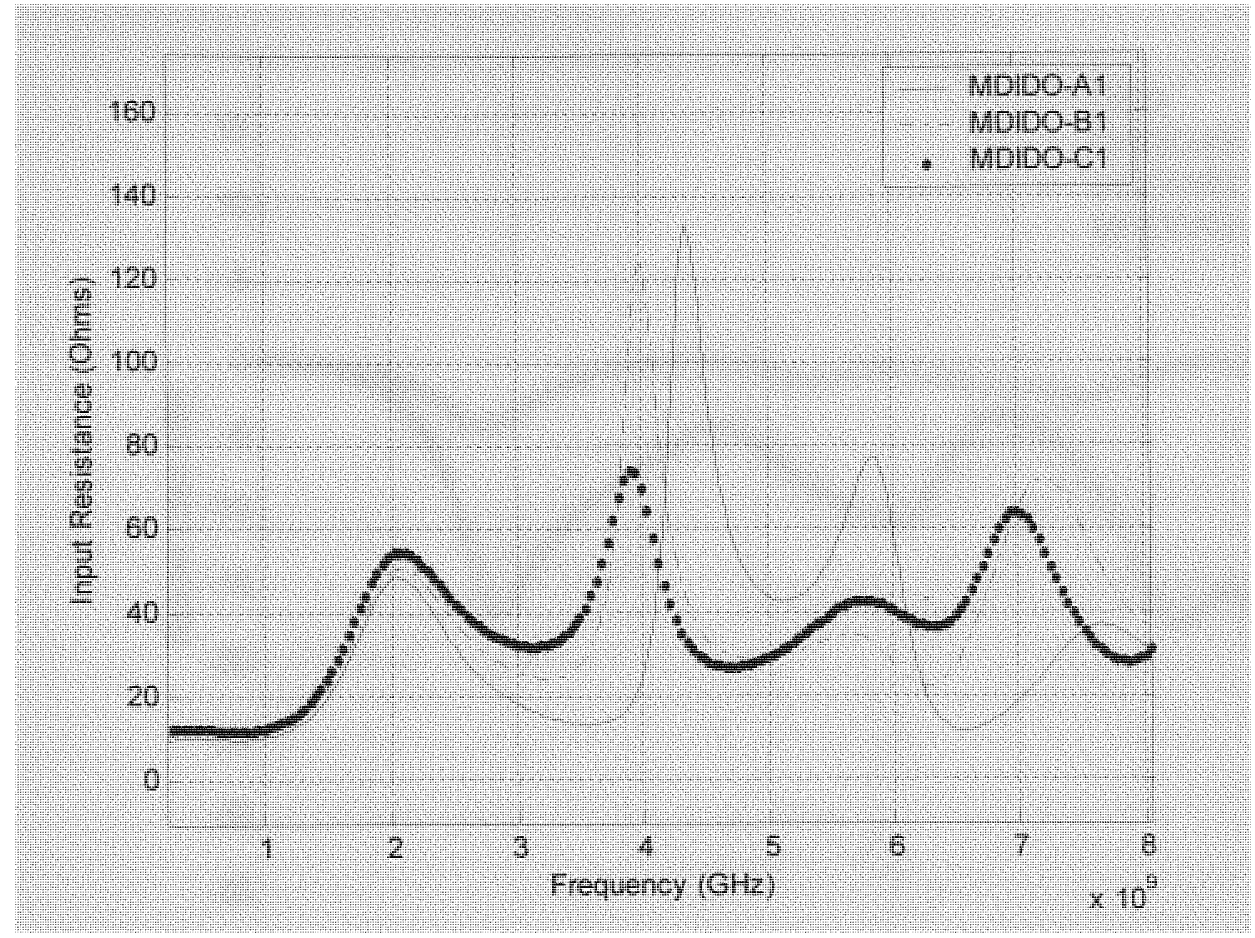

Fig. 92. Frequency Vs. input resistance for MDIDO-A1, MDIDO-B1, MDIDO-C1.

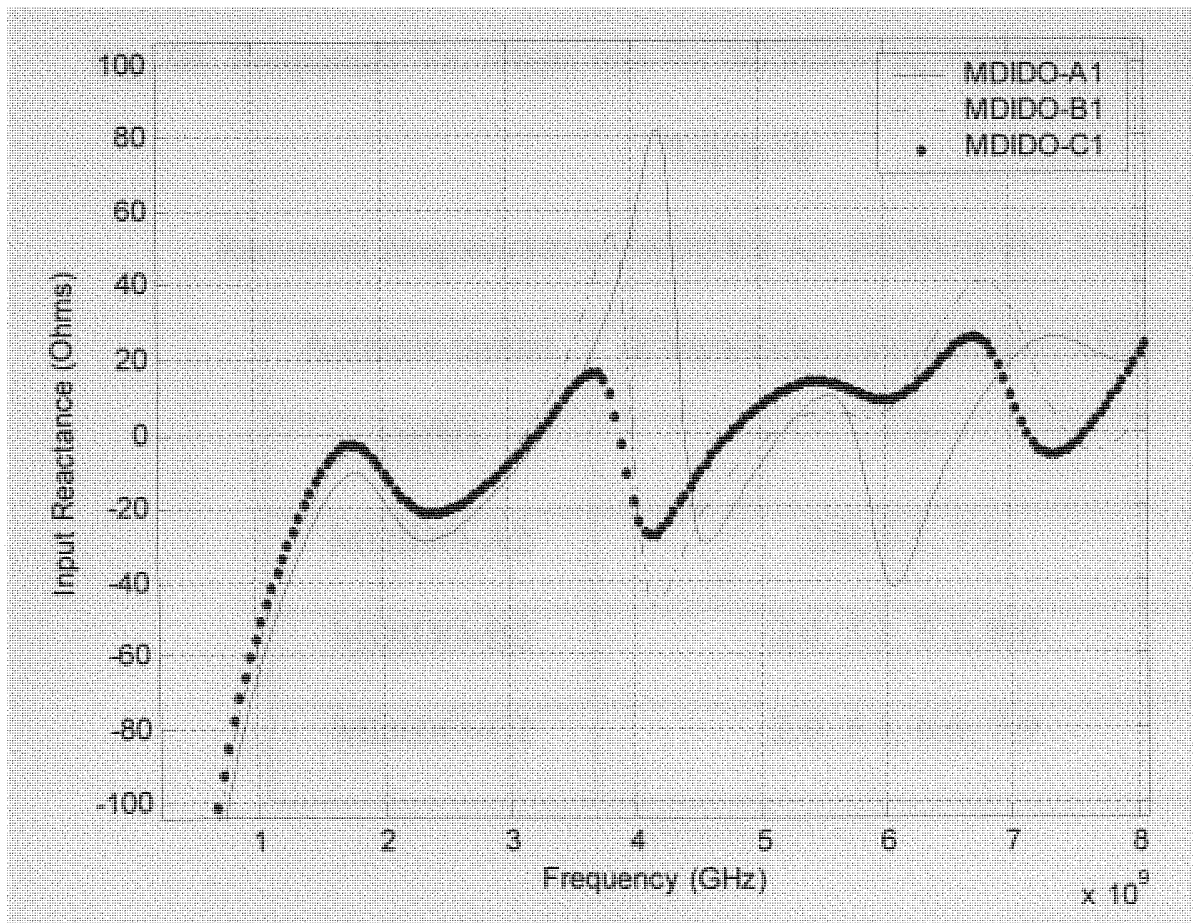

Fig. 93. Frequency Vs. input reactance for MDIDO-A1, MDIDO-B1, MDIDO-C1. 


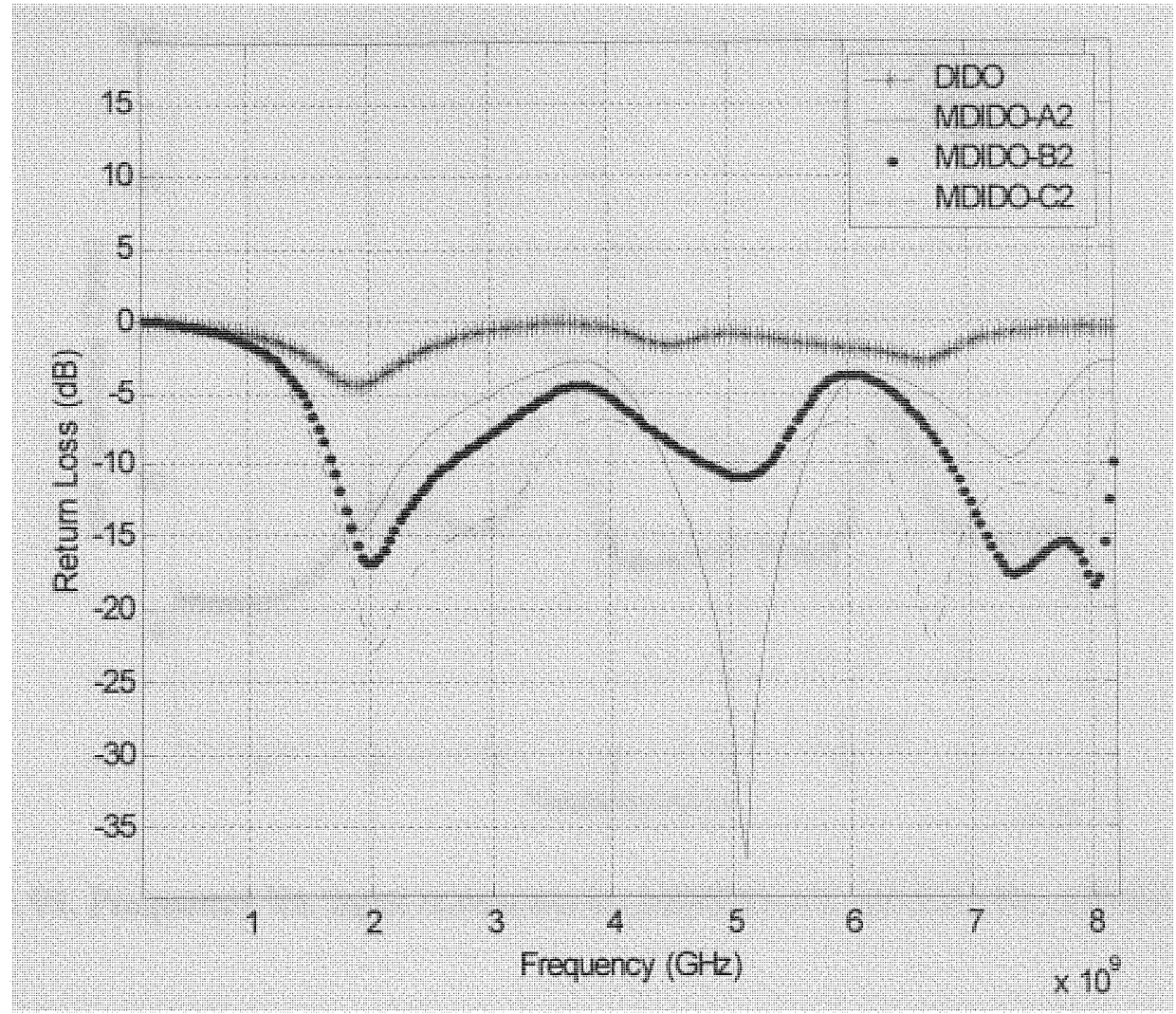

Fig. 94. Frequency Vs. return loss for MDIDO-A2, MDIDO-B2, MDIDO-C2.

Table 10

Resonant frequencies of MDIDO-A2, MDIDO-B2, MDIDOC2

\begin{tabular}{|c|c|}
\hline Antenna model & Frequencies of resonance $(\mathrm{GHz})$ \\
\hline DIDO & 1.9 \\
\hline MDIDO-A2 & $2.0,5.1,7.3$ \\
\hline MDIDO-B2 & $2.0,5.1,7.3,8.0$ \\
\hline MDIDO-C2 & $2.0,5.1,6.6,7.9$ \\
\hline
\end{tabular}




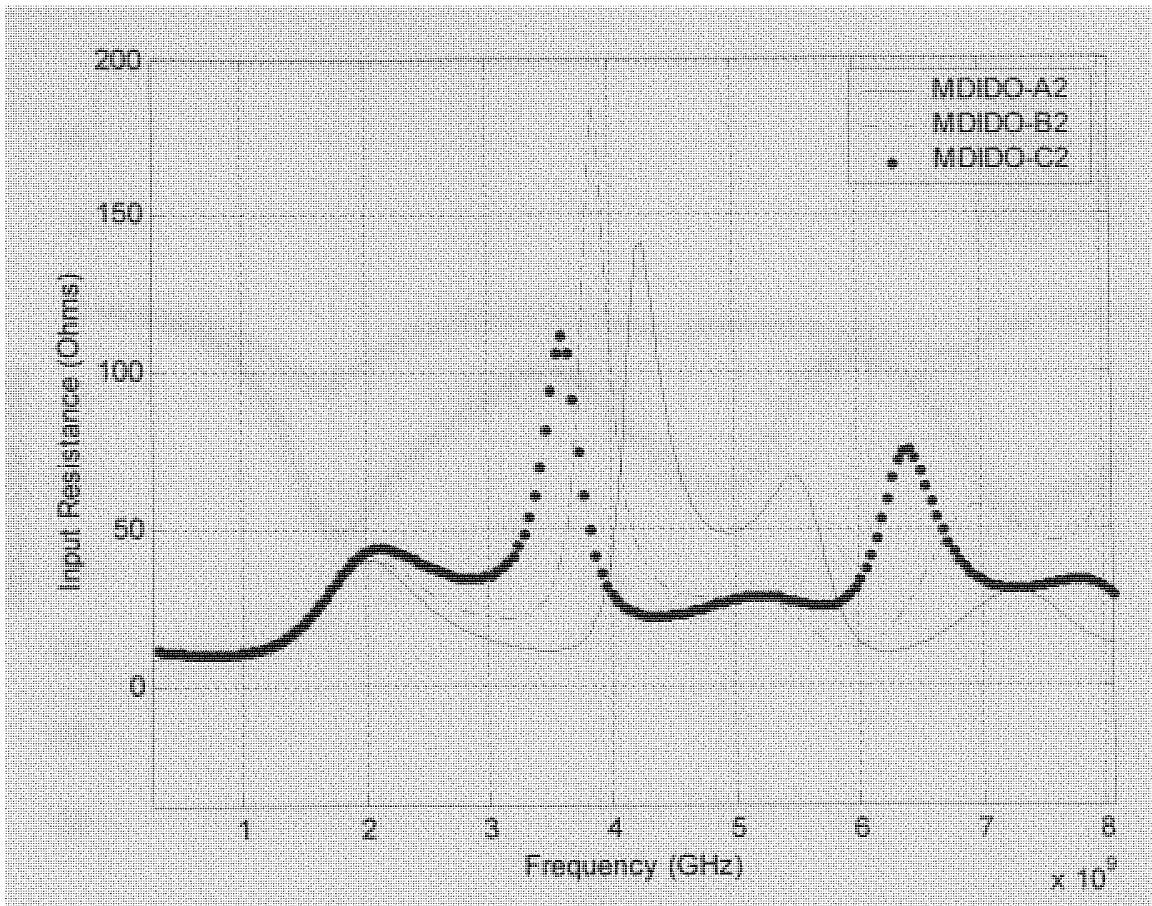

Fig. 95. Frequency Vs. input resistance for MDIDO-A2, MDIDO-B2, MDIDO-C2.

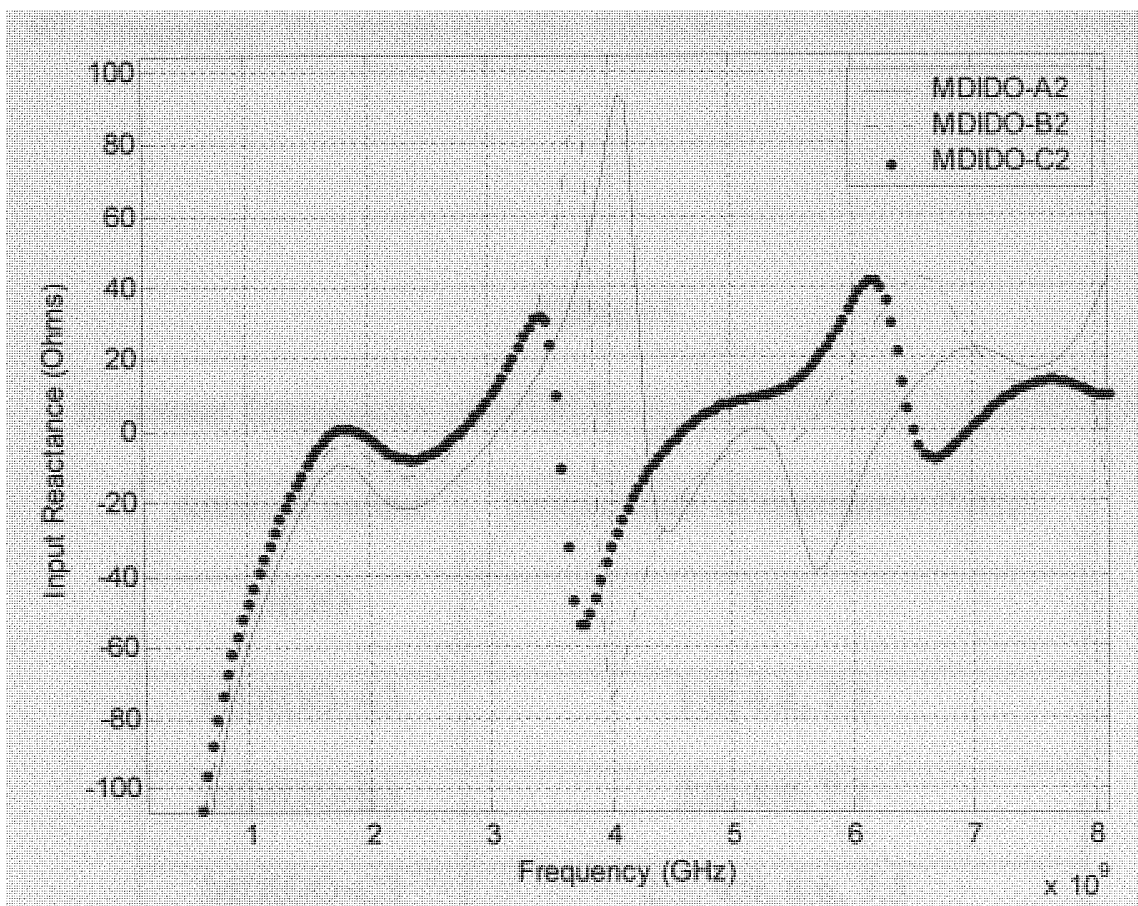

Fig. 96. Frequency Vs. input reactance for MDIDO-A2, MDIDO-B2, MDIDO-C2. 


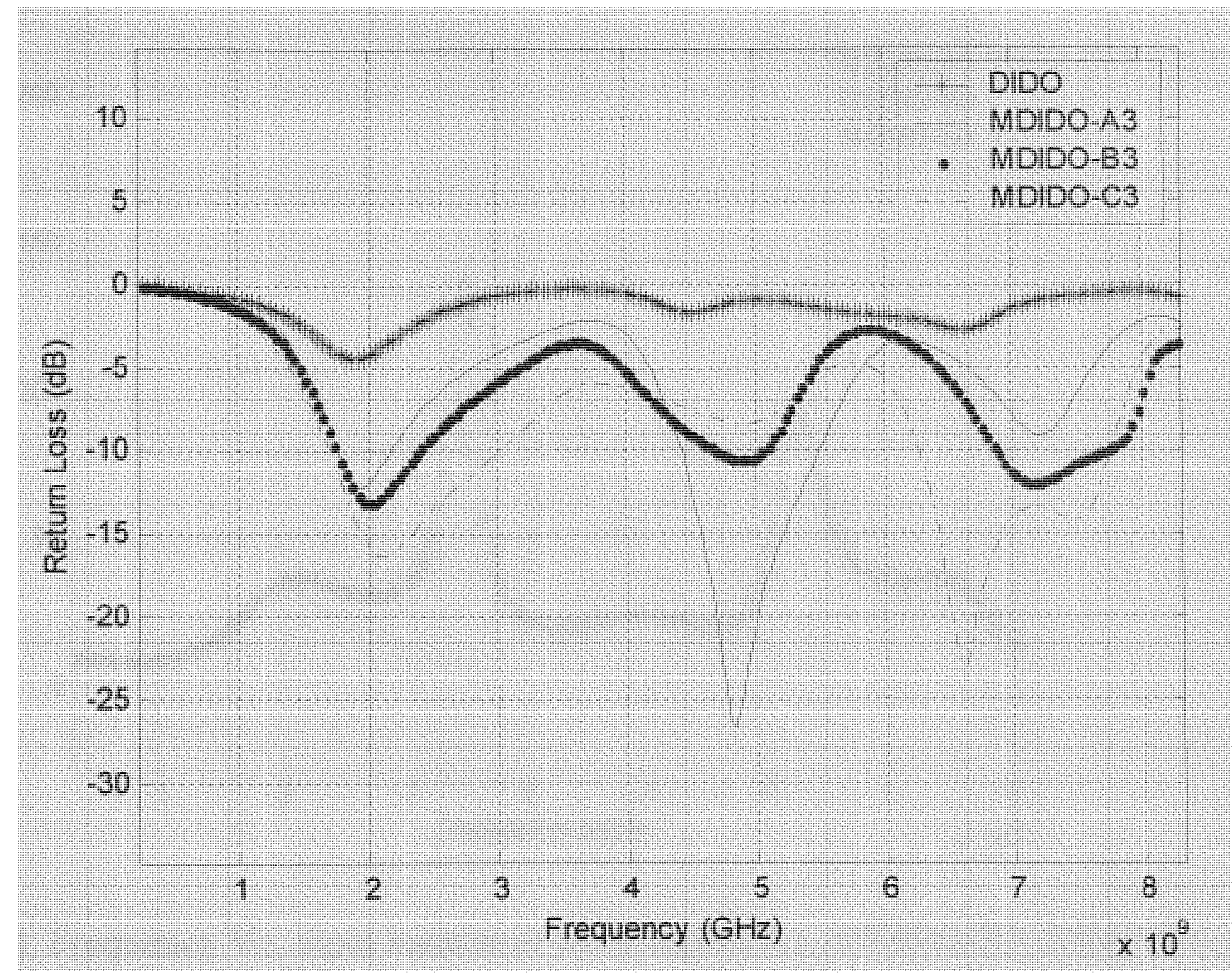

Fig. 97. Frequency Vs. return loss for MDIDO-A3, MDIDO-B3, MDIDO-C3.

Table 11

Resonant frequencies of MDIDO-A3, MDIDO-B3, MDIDO-C3

\begin{tabular}{|c|c|}
\hline Antenna model & Frequencies of resonance $(\mathrm{GHz})$ \\
\hline DIDO & 1.9 \\
\hline MDIDO-A3 & $1.9,4.8,7.2$ \\
\hline MDIDO-B3 & $2.0,4.9,7.1$ \\
\hline MDIDO-C3 & $2.1,4.9,6.6,7.5$ \\
\hline
\end{tabular}




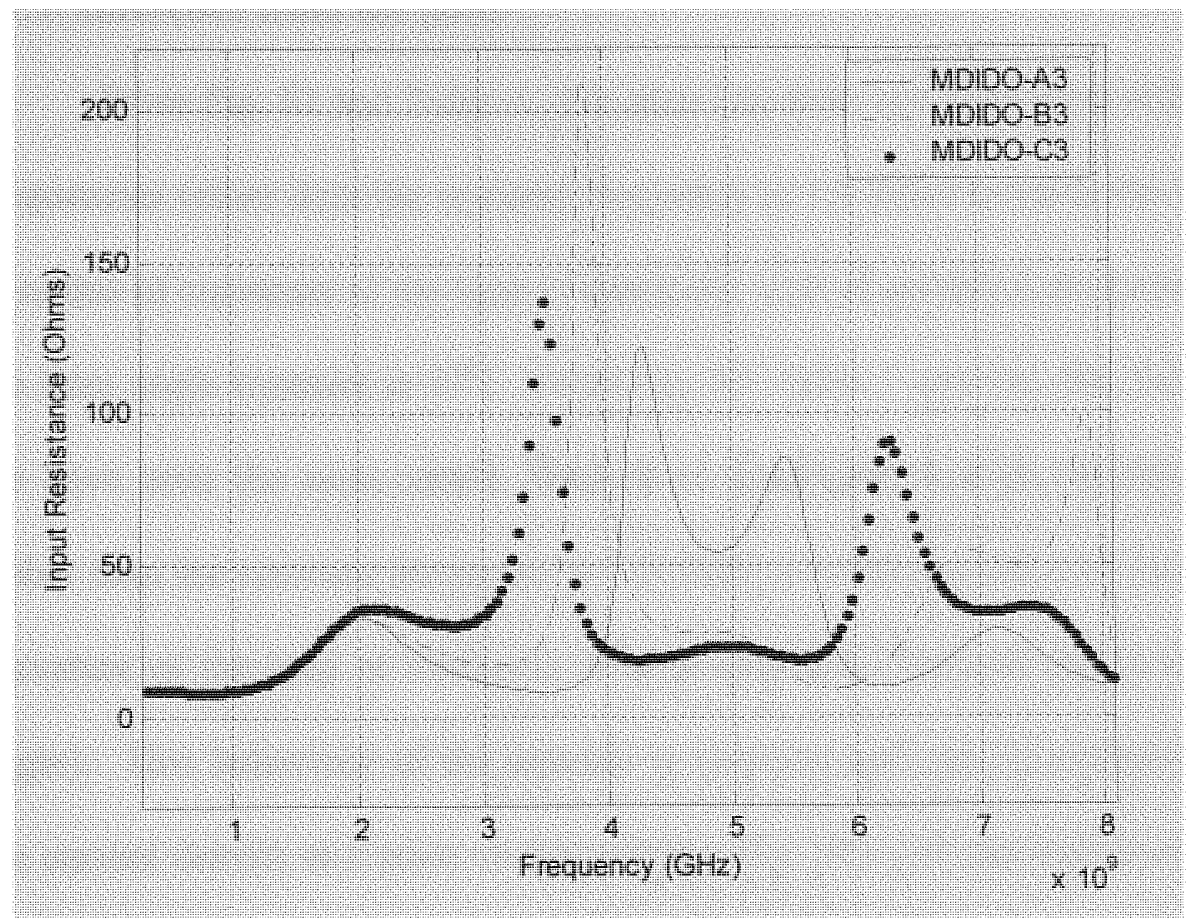

Fig. 98. Frequency Vs. input resistance for MDIDO-A3, MDIDO-B3, MDIDO-C3.

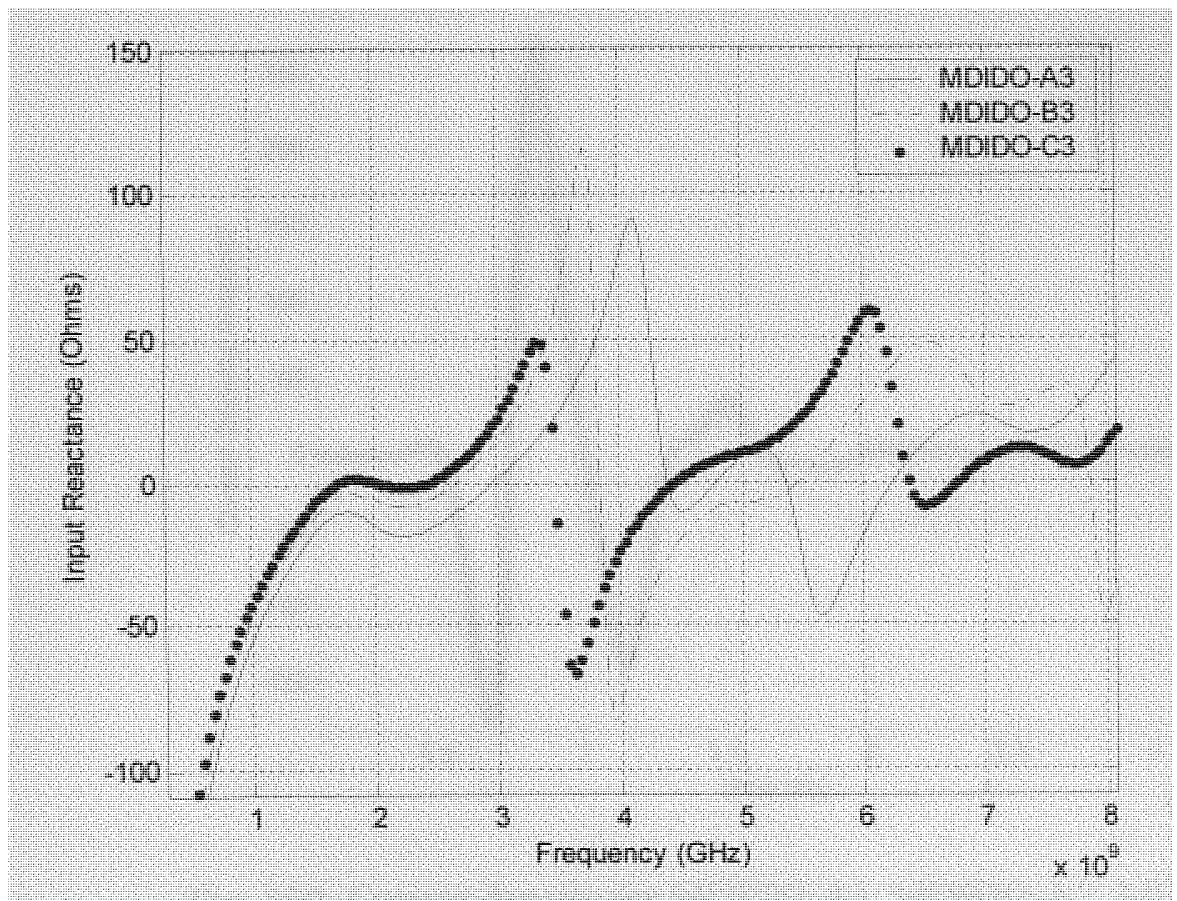

Fig. 99. Frequency Vs. input reactance for MDIDO-A3, MDIDO-B3, MDIDO-C3. 


\subsubsection{Inference from the plots}

From Fig. 82 and Table 6, it is seen that there is a significant shift in the resonant frequencies (other than the resonant frequency at $1.9 \mathrm{GHz}$ ) towards the left with increase in radius of curvature of the base of triangular arms of modified diamond dipole (keeping the flare angle constant at $27^{\circ}$ ). From Fig. 85, Table 7 and Fig. 88, Table 8 respectively we have seen the same result. There is a significant shift in the resonant frequencies (other than the resonant frequency at $1.9 \mathrm{GHz}$ ) towards the left with increase in radius of curvature of the base of triangular arms of modified diamond dipole (for constant flare angle). This behaviour of the antenna is expected and can be explained by considering the current density at a point on the antenna. Fig. 100-102 show plots of current densities of various modified diamond dipole antenna models.

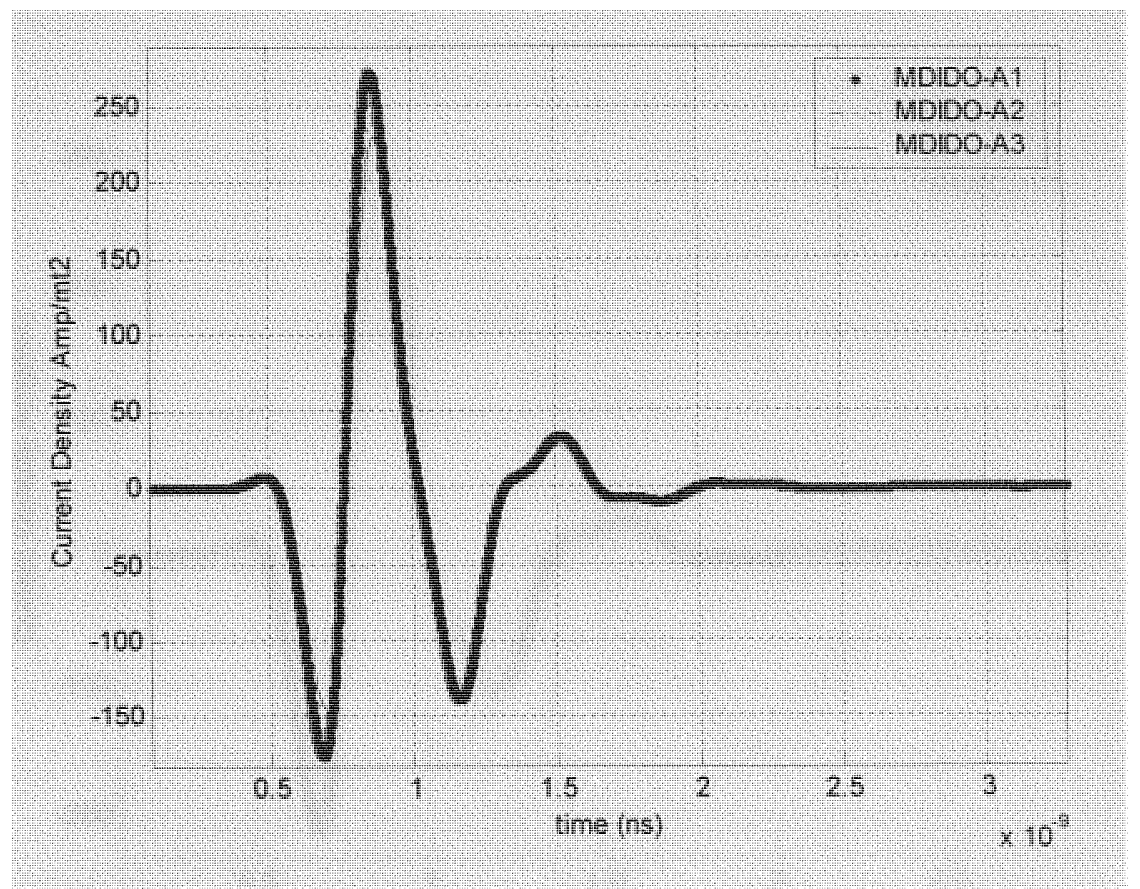

Fig. 100. Current density for MDIDO-A1, MDIDO-A2, MDIDO-A3. 


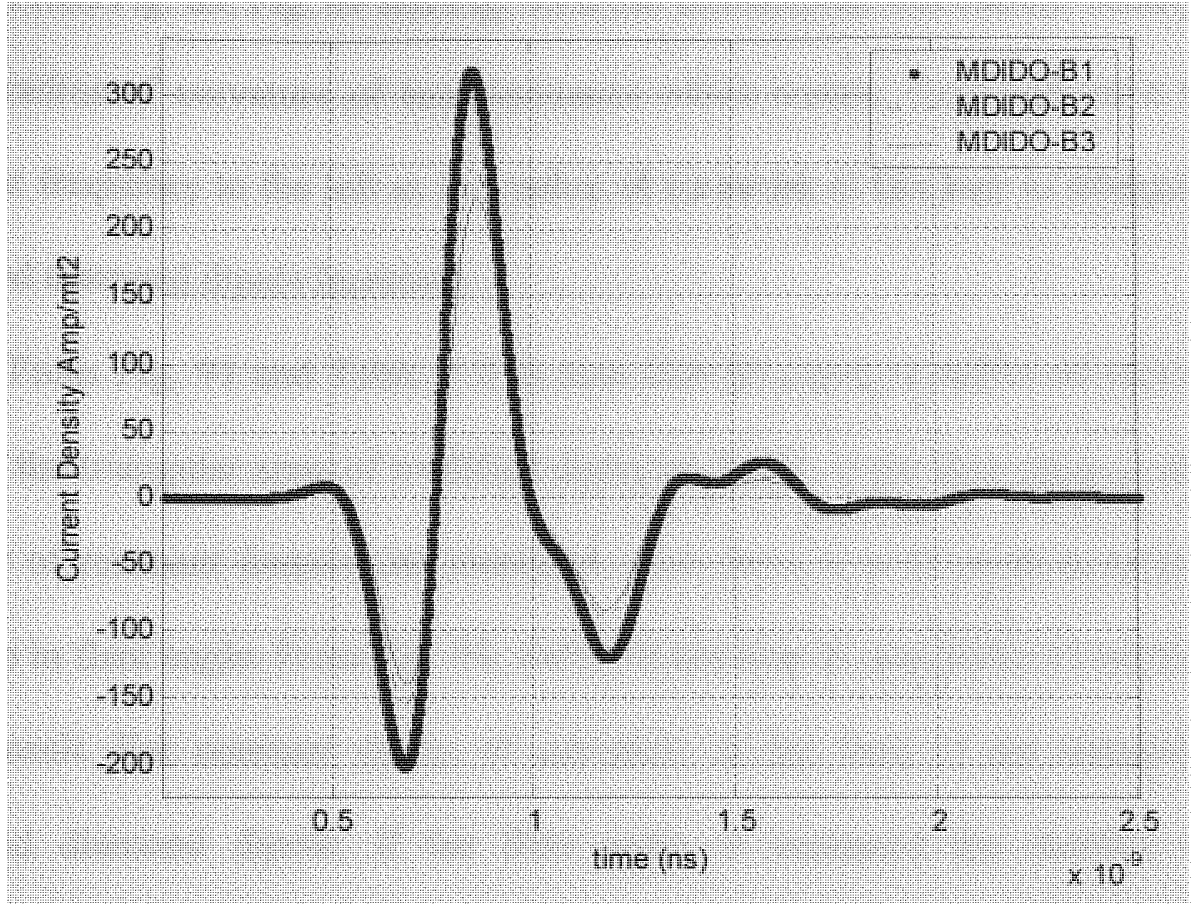

Fig. 101. Current density for MDIDO-B1, MDIDO-B2, MDIDO-B3.

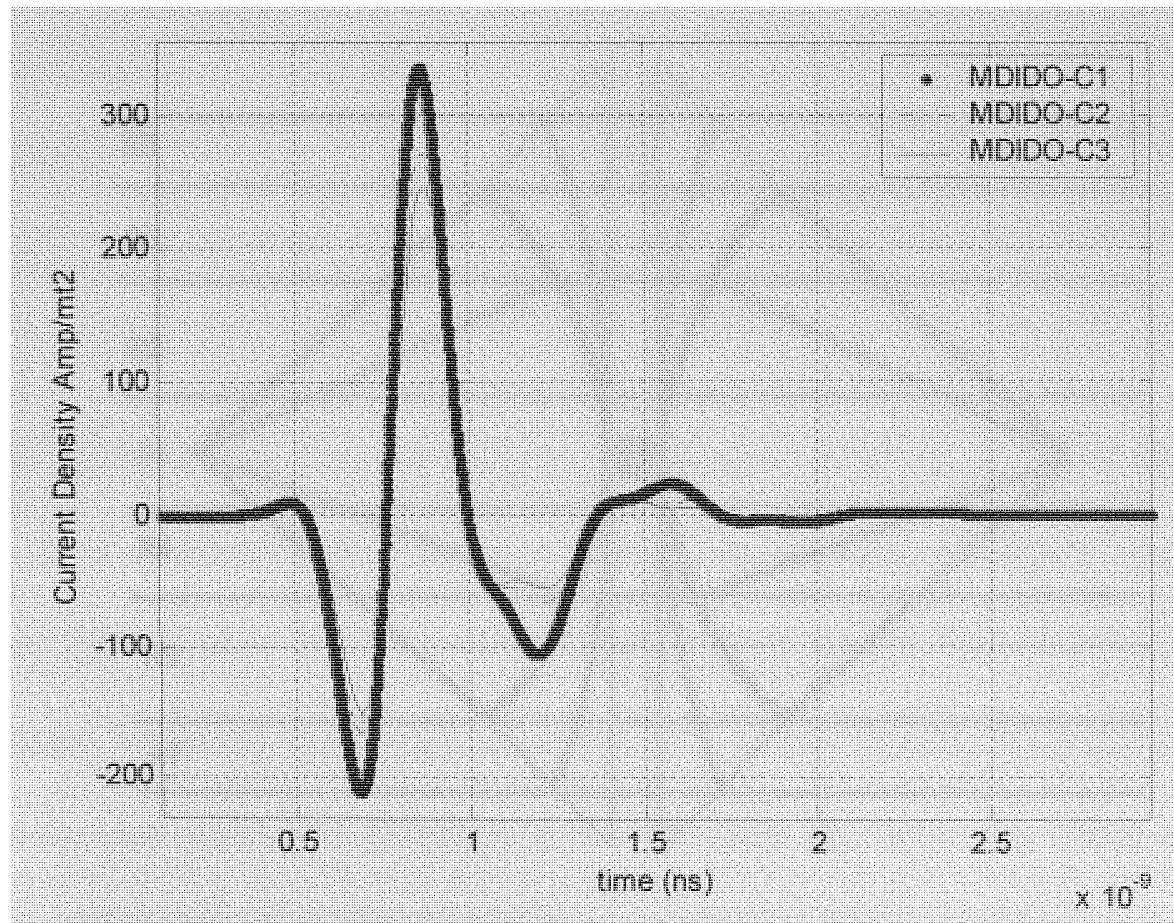

Fig. 102. Current density for MDIDO-C1, MDIDO-C2, MDIDO-C3. 
From Fig. 88, Fig. 89 and Fig. 90 it can be observed that current density decreases as the radius of curvature of base increases which means, there will be a shift in the resonant frequency to the left as the radius of curvature of the base of triangular arms of modified diamond dipole increases.

From Fig. 91 and Table 9 it is seen that for constant radius of curvature of the base of the triangular arms of modified diamond dipole, if the flare angle is increased the number of resonant frequencies increased. Also there is a decrease in return loss with increase in flare angle. From Fig. 94, Table 10 and Fig. 97 and Table 11 we have seen the same result. For constant radius of curvature of the base of the triangular arms of modified diamond dipole, if the flare angle is increased the number of resonant frequencies increased. Also there is a decrease in return loss with increase in flare angle.

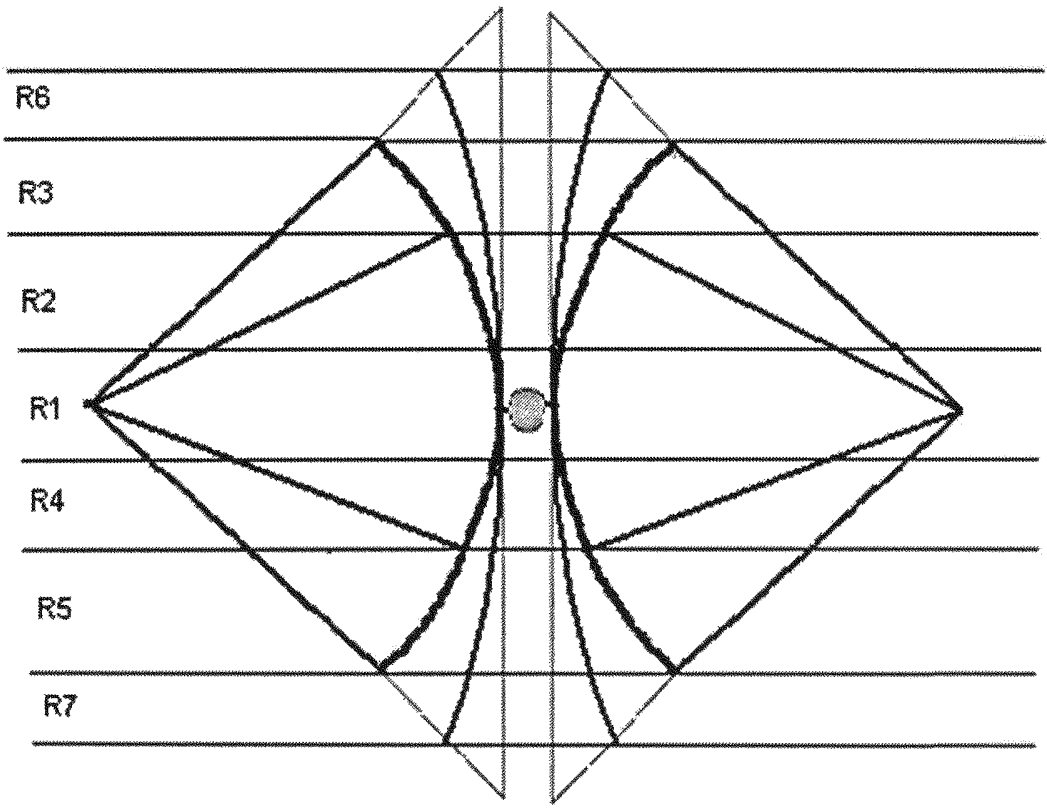

Fig. 103. Figure showing how arc length increases with flare angle. 
This behavior of the antenna can be explained by considering the Fig. 103. As the flare angle increases, the arc length increases and hence the antenna can be divided into more number of regions (like R1, R2 etc.) and hence the number of resonant frequencies increase because of coupling between the regions.

There is not much shift in the resonant frequency at $2 \mathrm{GHz}$ because the region $\mathrm{R} 1$ is approximately the same for both diamond dipole and modified diamond dipole.

\subsection{Observations made from various plots of slot loaded modified diamond dipole}

For clarity the designs names of slot loaded modified diamond dipole are provided here again.

Table 12

Information about the slot loaded modified diamond dipole antenna models

\begin{tabular}{|c|c|c|}
\hline Antenna model & Slot width (D1 in mm) & Distance of slot from feed (D2 in mm) \\
\hline SLMDIDO-A & 8 & 1 \\
\hline SLMDIDO-B & 6 & 3 \\
\hline SLMDIDO-C & 4 & 5 \\
\hline
\end{tabular}

Fig. 105 shows return loss for various slot loaded modified diamond dipole antenna models. It is observed that there is a shift in the resonant frequency as the slot size increases. The distance of the slot from the feed plays a major role in the amount of shift the resonant frequency undergoes. Here the shift in the resonant frequency is a function of both the slot width and distance of the slot from the feed. We observe that as D2, distance of the slot from the feed decreases, there will be an increase in the current density in the region D2, and hence there will be more shift in the resonant frequency to 
the left. Also as the slot size increase the current have to travel longer to traverse the dipole arms.

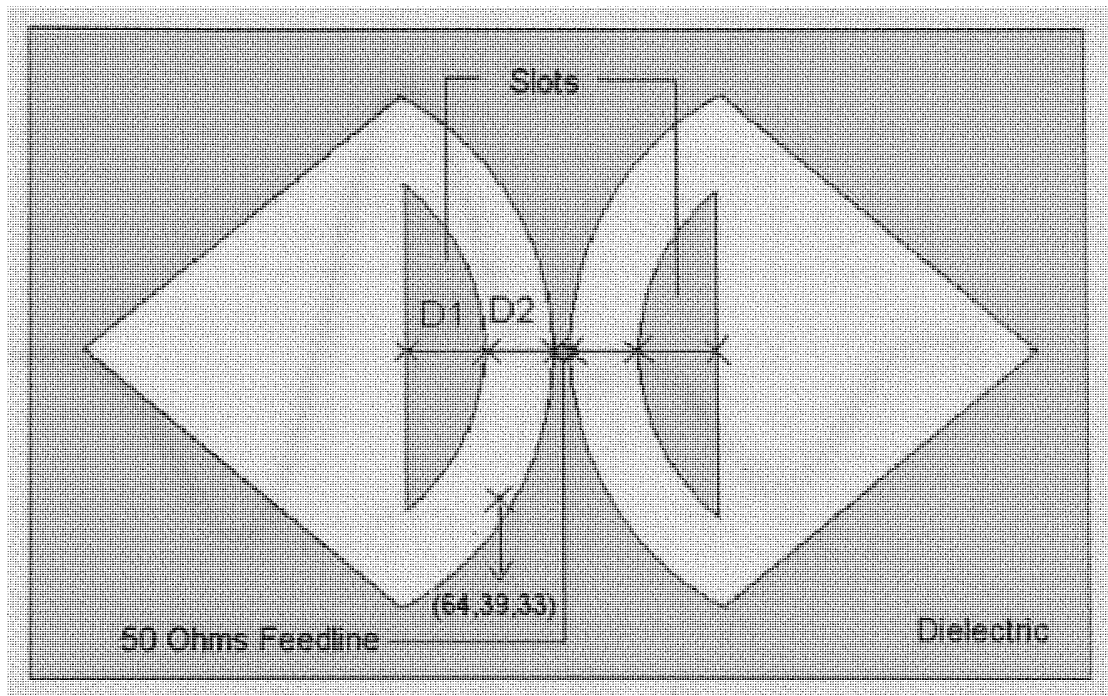

Fig. 104. Slot loaded modified diamond dipole with regions D1 and D2.

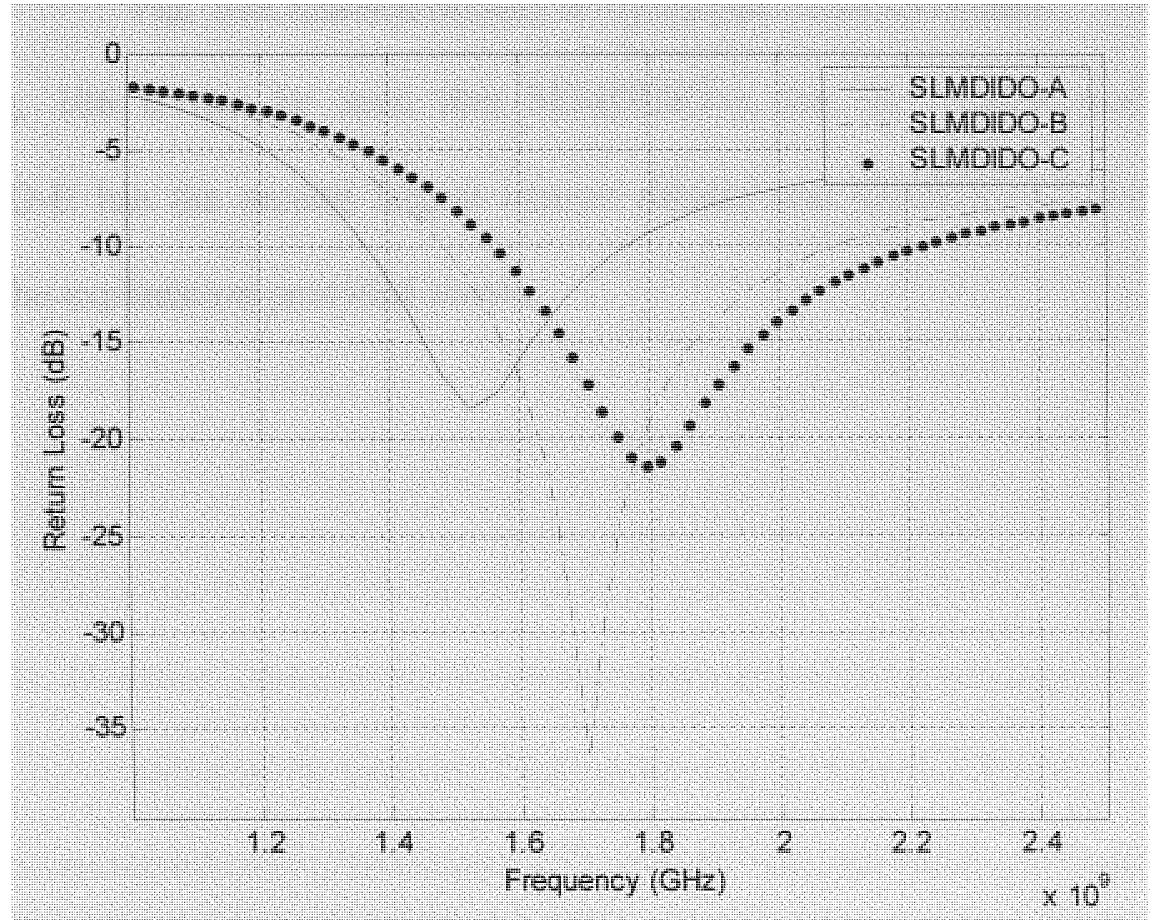

Fig. 105. Frequency Vs. return loss of slot loaded modified diamond dipole models. 




Fig. 106. Current density of various SLMDIDO models.

From Fig. 106 it can be seen that SLMDIDO-A has the largest current density, SLMDIDO-B has the second largest current density and SLMDIDO-C the least. This supports the explanation provided above. 


\section{CHAPTER 9}

\section{DESIGN EXAMPLE}

\subsection{Design}

From Chapter VIII, section 8.1.1, we can say that the return loss decreases as the radius of curvature decreases. The reason behind that is that for small radius of curvatures there is a smoother transition from the arc shaped base to the sides of the triangular arms of modified diamond dipole. Hence for a given flare angle, the smallest radius of curvature of the base gives the lowest return loss. The smallest radius of curvature for a given flare angle is nothing but the radius of In-circle of the triangular arm as shown in Fig. 107.

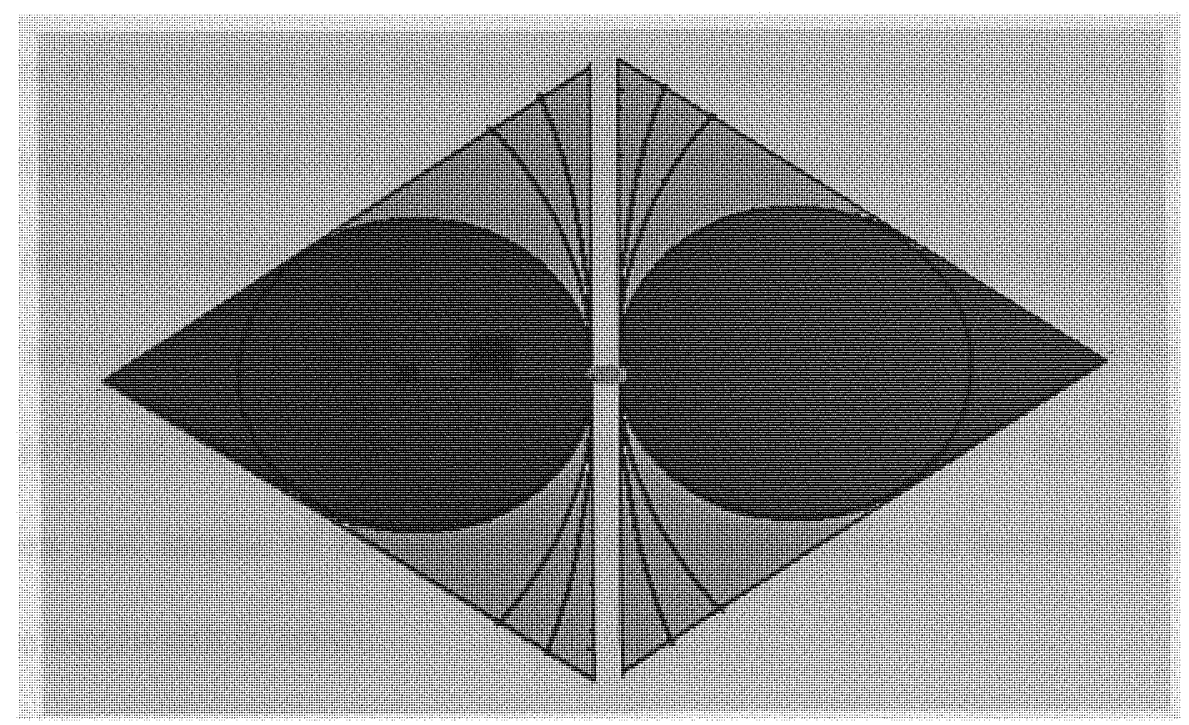

Fig. 107. Modified diamond dipole with base having radius of curvature equal to the radius of the incircle of triangular arm of diamond dipole.

Three antenna models with flare angles $27^{\circ}, 35^{\circ}, 45^{\circ}$ and the radius of curvatures of base equal to the radius of in-circles of triangular arms of respective antennas have been developed. Table 13 provides the information about the antenna models. 
Table 13

Information about the most efficient antenna models in terms of return loss

\begin{tabular}{|c|c|c|}
\hline Antenna model & Semi-flare angle in degrees & Radius of curvature in mm \\
\hline MDIDO-EFF1 & 27 & 12 \\
\hline MDIDO-EFF2 & 35 & 13 \\
\hline MDIDO-EFF3 & 45 & 15 \\
\hline
\end{tabular}

The radius of curvatures are rounded to the nearest integer. Fig. 108 show a comparison of return loss of the antenna models shown in table 2. From Fig. 108 the return loss for modified diamond dipole with flare angle $27^{\circ}$ has crossed the $-20 \mathrm{~dB}$ mark (for all the frequencies of resonance) and that for modified diamond dipole with flare angle $35^{\circ}$, return loss has crossed the $-15 \mathrm{db}$ mark (for all the frequencies of resonance) and that for modified diamond dipole with flare angle $45^{\circ}$, the return loss has crossed the $-25 \mathrm{~dB}$ mark (for almost all the frequencies of resonance). The reason for obtaining four resonant frequencies for all the flare angles can be attributed to the length of the base arc. But it can be seen that inspite of the return loss at the frequencies of resonance is small the percentage bandwidth is small.

It can be observed that as the flare angle $\alpha \rightarrow 180, R \rightarrow 18 \mathrm{~mm}$ (twice the length of the arm of modified diamond dipole) and in such case the modified diamond dipole would transform into circular dipole with each arm having a radius of $18 \mathrm{~mm}$. This transformation of modified diamond dipole into circular dipole is shown in Fig. 109. The sides of triangular arms of modified diamond dipole are represented as a1, b1, a2, b2, c1, c2. Fig. 110 shows the return loss of circular dipole. 


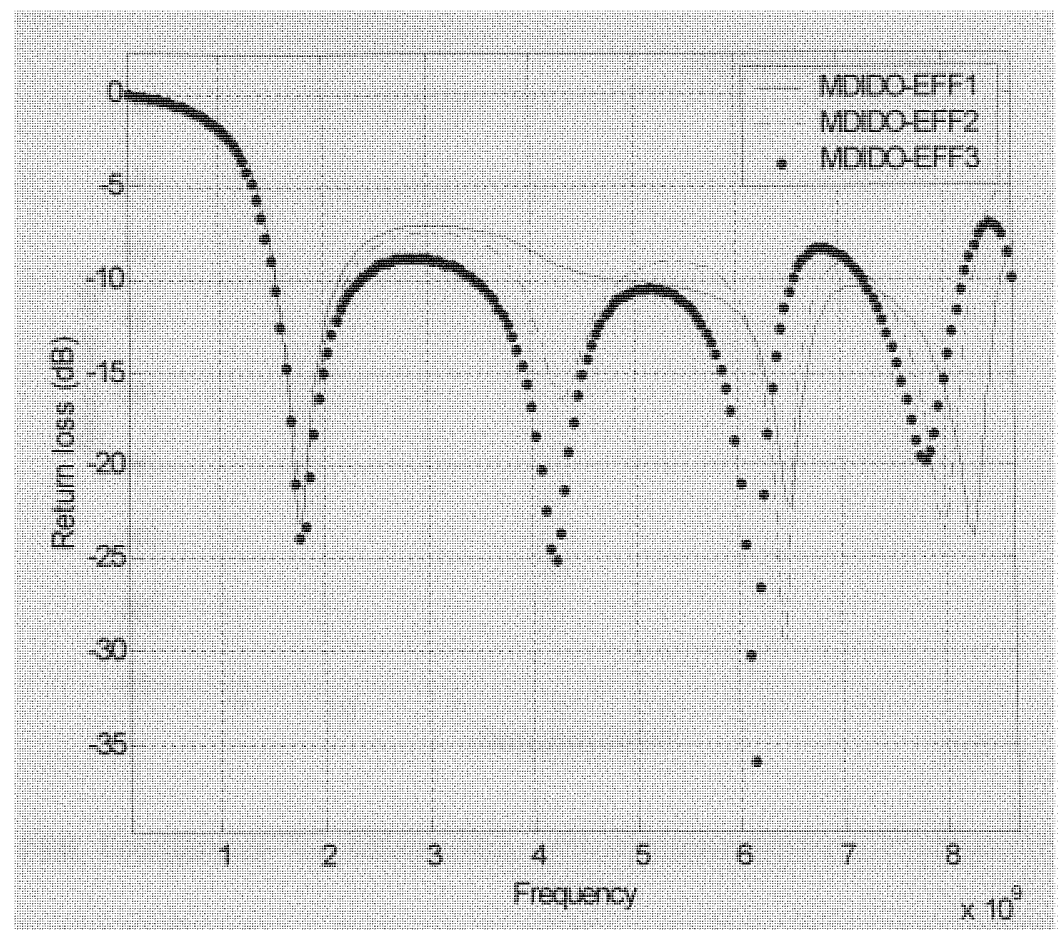

Fig. 108. Comparison of frequency Vs. return loss for various antenna models.

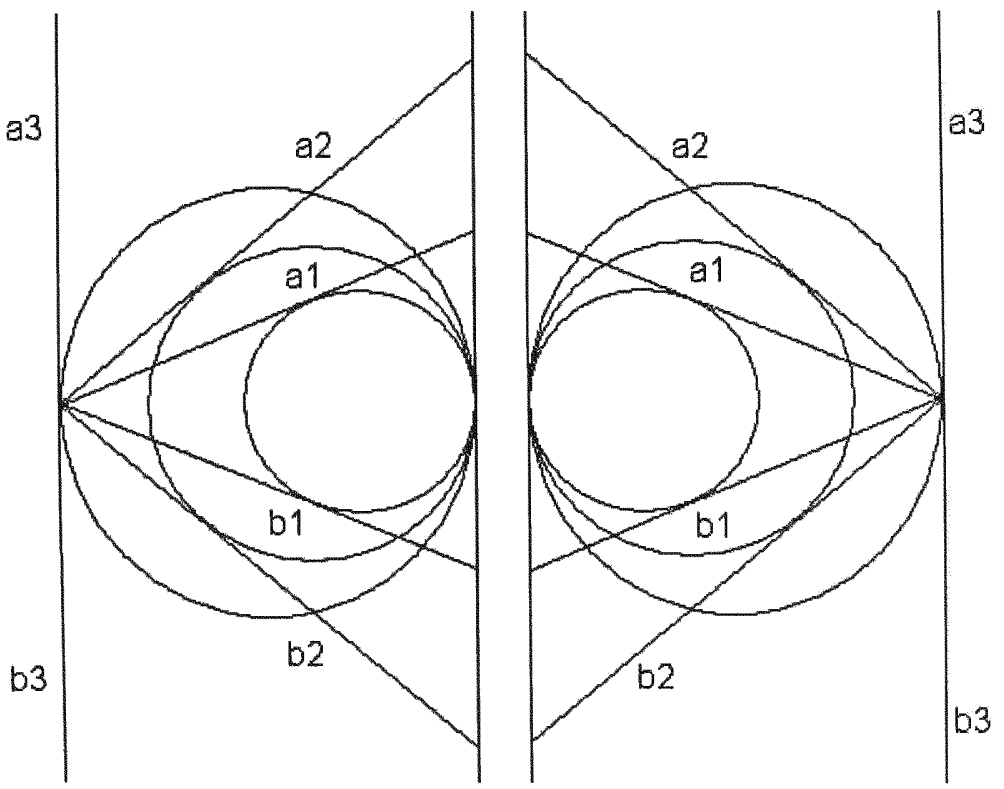

Fig. 109. Modified diamond dipole to circular dipole transformation. 


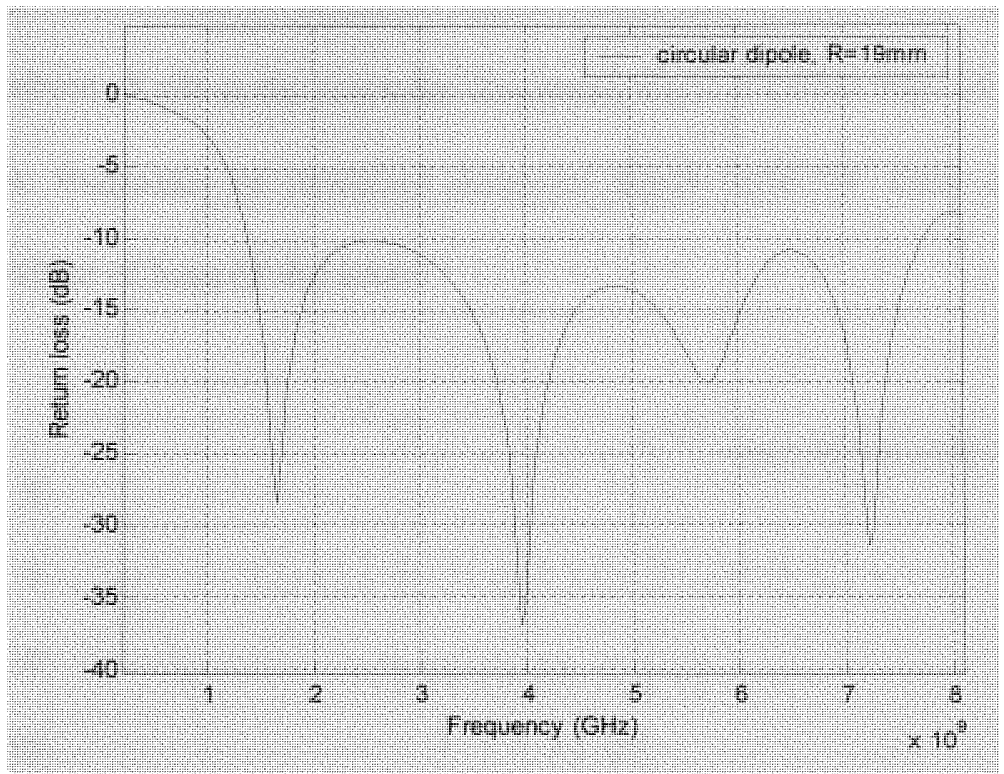

Fig. 110. Frequency Vs. return loss of a circular dipole of radius $\mathrm{R}=19 \mathrm{~mm}$.

We have selected $90^{\circ}$ to be a flare angle of the modified diamond dipole to study. The reason is that, as we have studied that as the radius of curvature increases (or with an increase in the arc length) the return loss increases which happens if we increase the flare angle. Also for very large flare angles $(\propto>>90)$ the shape of modified diamond dipole approaches the circular dipole (from Fig. 109). Hence $90^{\circ}$ (which is the angle in a semi circle) is chosen as the angle of interest. Hence the antenna with flare angle $90^{\circ}$, is tested for various radius of curvatures of the base of triangular arms. A value of $20 \mathrm{~mm}$ as radius of curvature yielded the largest percentage bandwidth of $137 \%$.

PercentageBandwidth $=\frac{2 \times\left(f_{2}-f_{1}\right)}{\left(f_{2}+f_{1}\right)} \times 100$

which can be written as follows by substituting the respective values

PercentageBandwidth $=\frac{2 \times(8.0-1.6)}{(8.0+1.6)} \times 100 \approx 137 \%$ 
The percentage bandwidth of diamond dipole based on $-10 \mathrm{~dB}$ mark is zero (since the return loss is about $-5 \mathrm{~dB}$ at the resonant frequency i.e. $1.9 \mathrm{GHz}$ ). Fig. 24. shows a plot of return loss Vs. frequency of modified diamond dipole with $90^{\circ}$ flare angle and $20 \mathrm{~mm}$ radius of curvature of base of the arms.

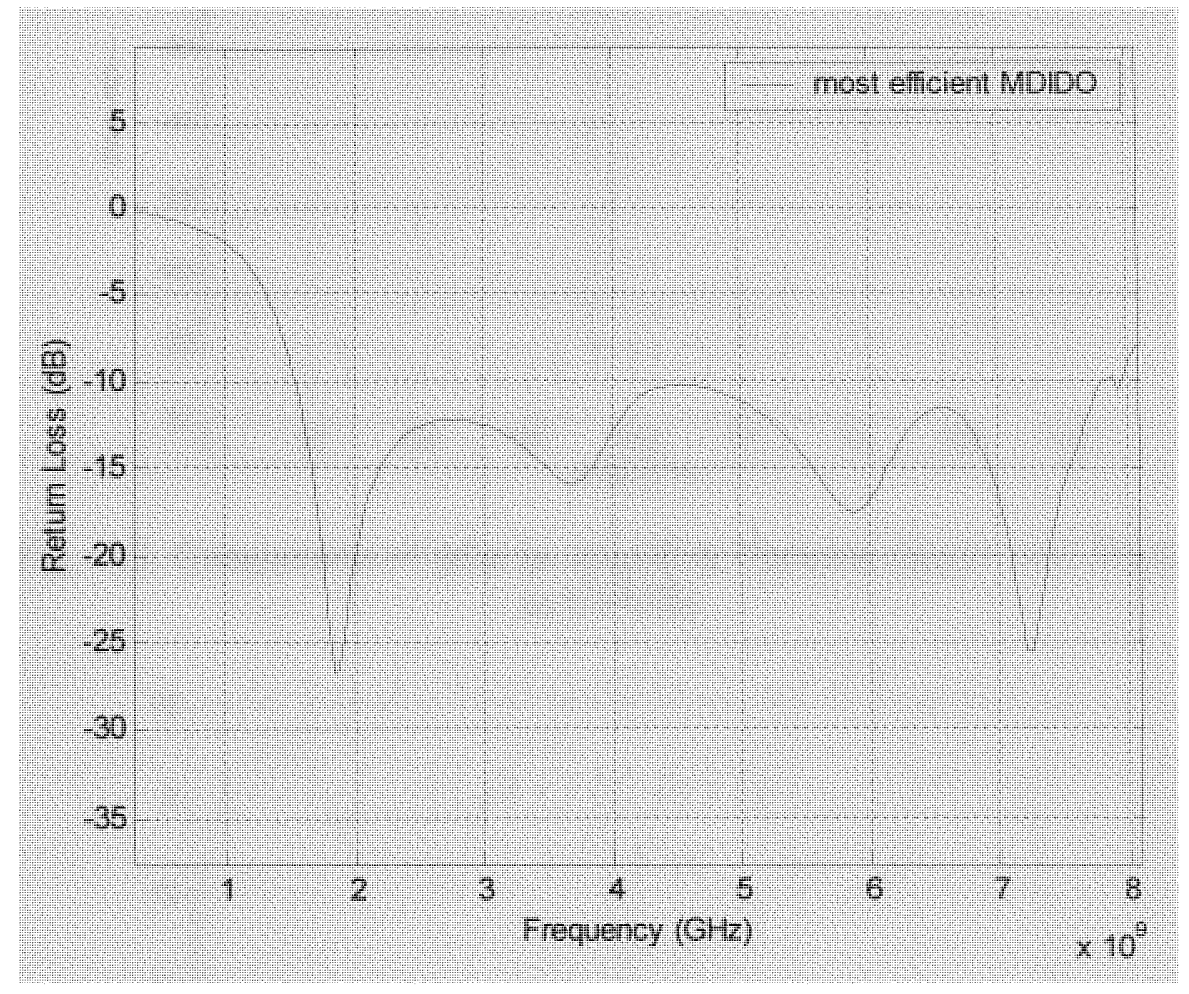

Fig. 111. Frequency Vs. return loss for most efficient modified diamond dipole.

Hence by appropriately selecting the flare angle and radius of curvature of the base of triangular arms of modified diamond dipole, the antenna can be designed to operate in selected frequency band. 


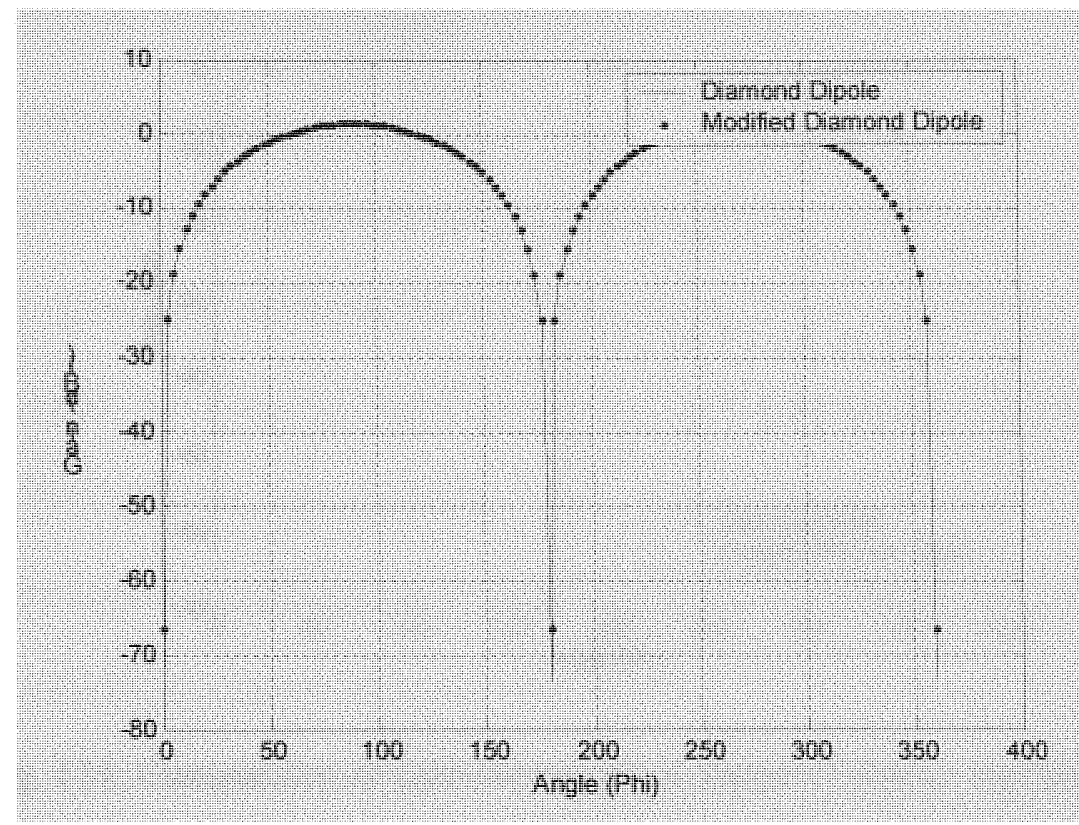

Fig. 112. Comparison of gain for modified diamond dipole and diamond dipole at $1.8 \mathrm{GHz}$.

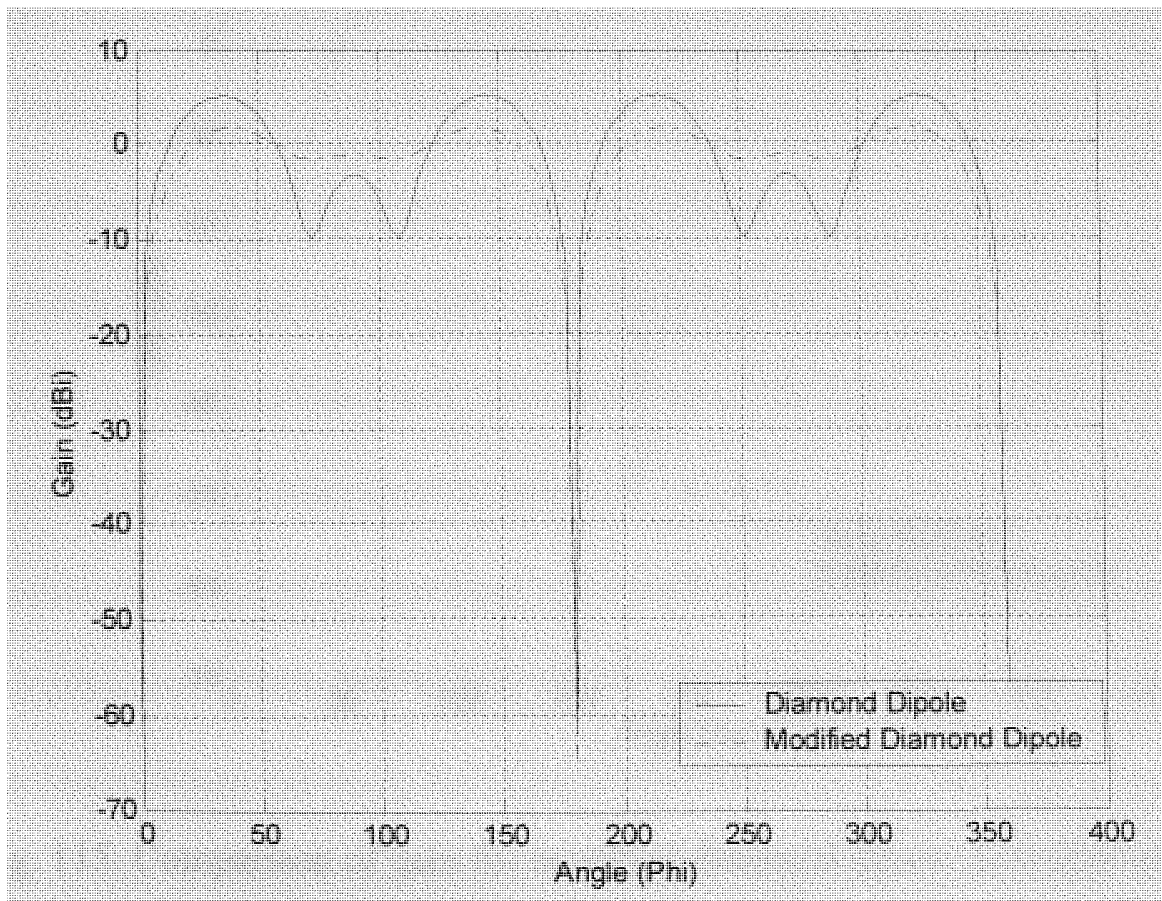

Fig. 113. Comparison of gain for modified diamond dipole and diamond dipole at $3.6 \mathrm{GHz}$. 


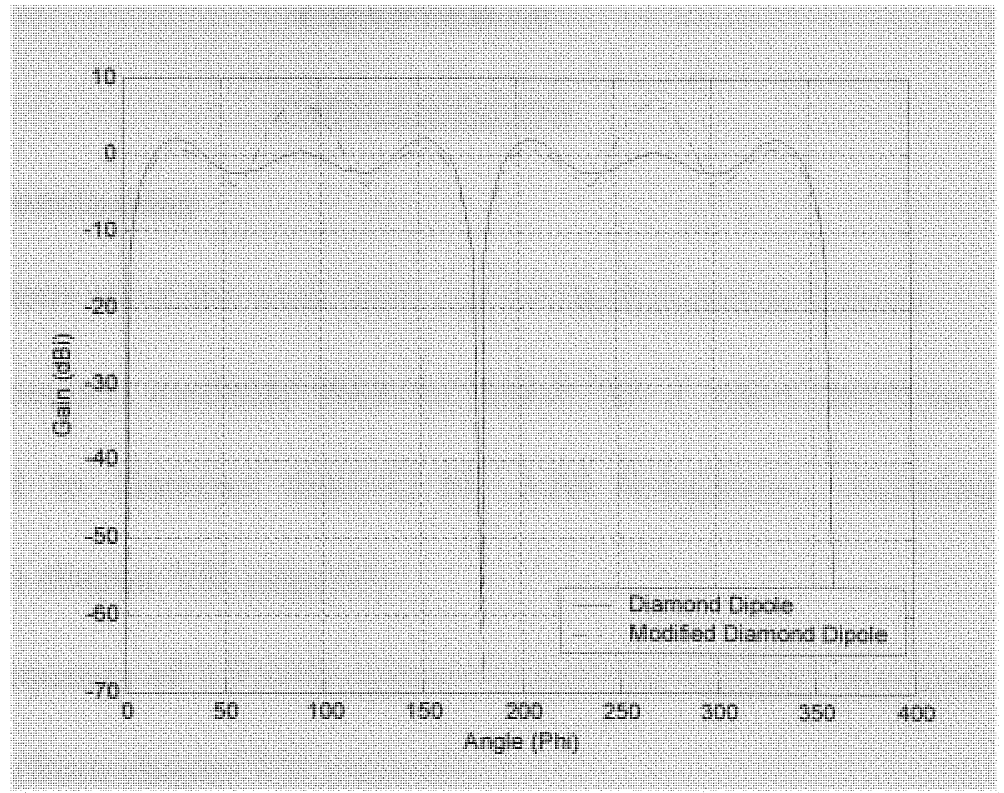

Fig. 114. Comparison of gain for modified diamond dipole and diamond dipole

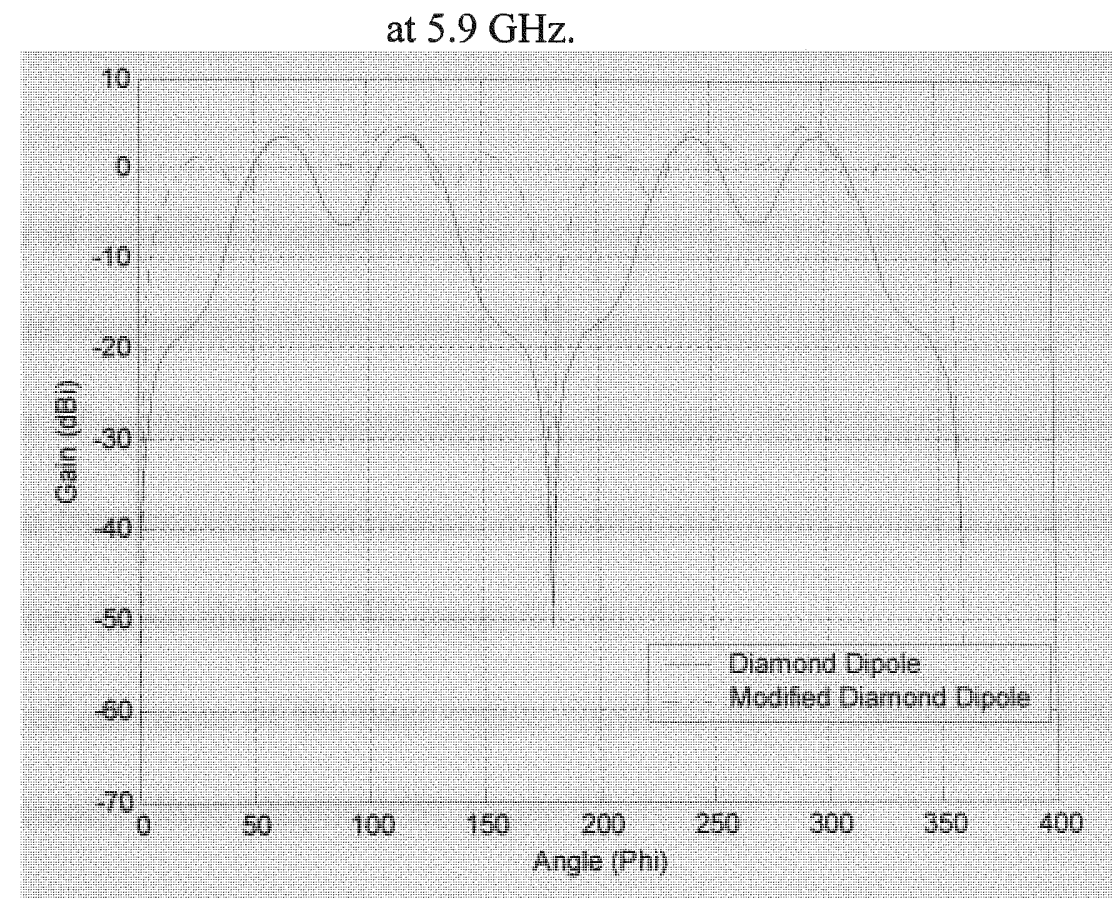

Fig. 115. Comparison of gain for modified diamond dipole and diamond dipole at $7.3 \mathrm{GHz}$. 


\section{CHAPTER 10 \\ CONCLUSIONS}

\subsection{Summary of conclusions}

The conclusions about the work done can be summarized as follows

- For a given geometry, modified diamond dipole has a lower reflection coefficient when compared to diamond dipole's (at most frequencies, including resonant frequency) (from chapter VII, page 69).

- The resonant frequency of $1.9 \mathrm{GHz}$ appears in both the modified diamond dipole and diamond dipole (from chapter VII, page 69).

- Modified diamond dipole offers more percentage bandwidth than diamond dipole. Hence modified diamond dipole can be used over a wider frequency range (chapter IX, page 96).

- As the flare angle of modified diamond dipole is increased, there is an increase in the number of resonant frequencies (from chapter VIII, pages $81,83,85$ ).

- As the flare angle of modified diamond dipole is increased, the reflection coefficient is decreased at the frequencies of resonance, indicating that more power is transferred to antenna from feed (from chapter VIII, pages $81,83,85$ ).

- When the radius of curvature of the base region (near the feed) is decreased, antenna radiate more efficiently (from chapter VIII, pages $75,77,79$ ).

- Least return loss is observed when the radius of curvature of the base region is equal to the radius of the in-circle of the triangular arms of modified diamond dipole (from chapter IX, page 95). 
- Modified diamond dipole is better matched to the 50 ohms feed than the diamond dipole(lower return loss than diamond dipole) (from chapter VII, page 69).

- When the radius of curvature is decreased there is a shift in the resonant frequency towards the left. Hence the resonant frequency is slightly decreased with decreasing radius of curvature (from chapter VIII, pages $75,77,70$ ).

- Resonant frequency at $1.9 \mathrm{GHz}$ is not affected with variations in either the flare angle or the radius of curvature of the base of modified diamond dipole or both (from chapter VIII, pages $75,77,79,81,83,85)$.

- Modified diamond dipole offer's more performance bandwidth (bandwidth over which the input resistance is fairly constant and input reactance is fairly constant and very close to zero) than diamond dipole (from chapter VII, pages 69, 70).

- When slots are introduced into the modified diamond dipole antenna, there is a shift in the resonant frequency (from chapter VII, page 73).

- The amount of shift in the resonant frequency depends on the slot size and the distance of the slot from the feed (from chapter VIII, pages 91, 92).

- The more the distance of the slot from the feed, the more the shift in resonant frequency (from chapter VIII, pages 91, 92).

- Also, as the slot dimension is increased there is a much wider shift in resonant frequency towards the left (from chapter VIII, pages 91, 92).

It can be summarized as modified diamond dipole offers better performance than diamond dipole in terms of reflection coefficient, percentage bandwidth and performance bandwidth. Slots introduce a shift in the resonant frequency to the left. 
The more the slot size and/or distance of the slot from the feed, the more the shift (to the left) in the resonant frequency. 


\section{CHAPTER 11}

\section{FUTURE WORK}

- An equation can be formulated relating the antenna dimensions flare angle, radius of curvature of the base of triangular arms, dielectric constant of the substrate, thickness of the substrate etc. with the percentage bandwidth which can be used in writing a genetic algorithm to get the most efficient geometry of the antenna with respect to percentage bandwidth.

- Many examples of modified diamond dipole can be constructed, simulated and the results (along with dimensions) used in training a neural network to get the required design to cover the required bandwidth using the neural network.

- Also a mechanical system can be designed (which can alter the slot sizes) to tune the antenna over a very wide frequency range. 


\section{REFERENCES}

[1] T. S. Rappaport, "Wireless Communications: Principles \& Practice," $2^{\text {nd }}$ Edition, Pretence Hall, Inc., 1996.

[2] K. Garg, "Ultra Wideband Antennas for Personal Communication Devices," M.S.Thesis, Dept. Electrical and Computer Eng, Florida Intl. University, Miami, 2001.

[3] US 47 CFR Part 15 Ultra-Wideband Operations FCC Report and Order, 22 April 2002, http://www.fcc.gov/Bureaus /Engineering_Technology/Orders/2002/ fcc02048.pdf.

[4] D.S. Filipovic, M. Nurnberger, and J.L. Volakis, "Ultra Wideband Slot Spiral With Dielectric Loading: Measurements and Simulations", IEEE Antennas and Propagation Society International Symposium, Vol. 3, pp. 1536-1539, July 2000.

[5] W.H. Darden and W.D. Burnside, "An Ultra-Wideband Antenna for Pulsed Applications”, Microwave Journal, vol. 36, pp. 136-143, Sep 1993.

[6] N. Yuan, J. He, and J. Mao, "A New Type of Ultra-Wideband Conical Antenna Ridged with a Novel Finline Radiator", International Journal of Infrared and Millimeter Waves, Vol. 19, No. 2, pp. 343-347, 1998.

[7] J.D.S. Langley, P.S. Hall, and P. Newham, "Novel Ultra Wide-bandwidth Vivaldi Antenna with low Crosspolarisation", Electronic Letters, vol. 29, No. 23, pp. 2004-2005, Nov 1993.

[8] A. Boryssenko, "Time Domain Studies of Ultra-Wideband Antennas", IEEE Canadian Conference on Electrical and Computer Engineering, vol. 1, pp. 95-100, May 1999.

[9] L. C. T. Chang and W. D. Burnside, "An Ultrawide-Bandwidth Tapered Resistive TEM Horn Antenna”, IEEE Trans. Antennas Propagat., vol. 48, No. 12, pp.18481857, Nov 1998.

[10] C. Nguyen, J. S. Lee, and J. S. Park, "Ultra-wideband Microstrip Quasi-horn Antenna", Electronic Letters, vol. 37, No. 12, pp.731-732, June 2001.

[11] R. Garg, P. Bhartia, I. Bahl, and A. Ittipiboon, "Microstrip Antenna Design Handbook", $2^{\text {nd }}$ Edition, Artech House, Inc: 2001.

[12] R. Garg and V. S. Reddy, "A Broad-Band Coupled-Strips Microstrip Antenna", IEEE Trans. Antennas Propagat., vol. 49, No. 9, pp.1344-1345, Sep 2001. 
[13] J. Romeu and J. Soler, "Generalized Sierpinski Fractal Multiband Antenna", IEEE Trans. Antennas Propagat., vol. 49, No. 8, pp.1237-1239, Aug 2001.

[14] J. Y. Sze and K. L. Wong, "Bandwidth Enhancement of a Microstrip-Line Fed Printed Wide-Slot Antenna", IEEE Trans. Antennas Propagat., vol. 49 No. 7, pp.1020-1023, July 2001.

[15] F. Yang, X. X. Zhang, X. Ye, and Y. R. Samii, "Wide-Band E-Shaped Patch Antennas for Wireless Communications", IEEE Trans. Antennas Propagat., vol. 49, No. 7, pp.1094-1100, July 2001.

[16] A. Hoorfar and A. Perrotta, "An Experimental Study of Microstrip Antennas on Very High Permittivity Ceramic Substrates and Very Small Ground Planes", IEEE Trans. Antennas Propagat., vol. 49, No. 4, pp.838-840, April 2001.

[17] C. A. Balanis, "Antenna Theory and Design", $2^{\text {nd }}$ Edition, John Wiley \& Sons, Inc., 2001.

[18] K. L. Wong, "Compact and Broadband Microstrip Antennas", $1^{\text {st }}$ Edition, John Wiley \& Sons, Inc., 2002.

[19] F. Yang, X. X. Zhang, X. Ye, and Y. R. Samii, "Wide-Band E-Shaped Patch Antennas for Wireless Communications", IEEE Trans. Antennas Propagat., vol. 49, No. 7, pp.1094-1100, July 2001.

[20] K. Kunz and R. J. Luebbers, "The Finite Difference Time Domain Method for Electromagnetics", CRC Press Inc., 1993.

[21] Remcomm Inc., "User's manual for XFDTD,"Version 5.0.4.7 Remcom Inc., July 1999.

[22] K. Siwiak, "Radiowave Propagation and Antennas for Personal Communications" $1^{\text {st }}$ Edition, Artech House, Inc., 1995.

[23] S. Desamsetty, E. Lule, T. M. Babij, K. Siwiak, "Analysis of Modified Diamond Dipole for Ultra Wideband Communications using FDTD Method", Antenna Application Symposium, pp. 100-120, Sep 2003. 